\title{
Measurement of beauty and charm production in deep inelastic scattering at HERA and measurement of the beauty-quark mass
}

\section{The ZEUS collaboration}

\author{
E-mail: m.wing@ucl.ac.uk
}

ABSTRACT: The production of beauty and charm quarks in ep interactions has been studied with the ZEUS detector at HERA for exchanged four-momentum squared $5<$ $Q^{2}<1000 \mathrm{GeV}^{2}$ using an integrated luminosity of $354 \mathrm{pb}^{-1}$. The beauty and charm content in events with at least one jet have been extracted using the invariant mass of charged tracks associated with secondary vertices and the decay-length significance of these vertices. Differential cross sections as a function of $Q^{2}$, Bjorken $x$, jet transverse energy and pseudorapidity were measured and compared with next-to-leading-order QCD calculations. The beauty and charm contributions to the proton structure functions were extracted from the double-differential cross section as a function of $x$ and $Q^{2}$. The running beauty-quark mass, $m_{b}$ at the scale $m_{b}$, was determined from a QCD fit at next-to-leading order to HERA data for the first time and found to be $m_{b}\left(m_{b}\right)=$ $4.07 \pm 0.14$ (fit) ${ }_{-0.07}^{+0.01}$ (mod. $)_{-0.00}^{+0.05}$ (param. $)_{-0.05}^{+0.08}$ (theo.) GeV.

KEYwords: Lepton-Nucleon Scattering, QCD, Jets, B physics

ARXIV EPRINT: 1405.6915 


\section{Contents}

1 Introduction $\quad 1$

2 Experimental set-up 2

3 Monte Carlo simulations 3

4 Theoretical predictions and uncertainties 4

5 Data selection 5

$\begin{array}{lll}6 & \text { Extraction of the heavy-flavour cross sections } & 6\end{array}$

$\begin{array}{llr}7 & \text { Systematic uncertainties } & 8\end{array}$

8 Cross sections $\quad 9$

$9 \begin{array}{ll}\text { Extraction of } F_{2}^{q \bar{q}} \text { and } \sigma_{\mathrm{r}}^{q \bar{q}} & 10\end{array}$

10 Measurement of the running beauty-quark mass 12

11 Conclusions $\quad 14$

The ZEUS collaboration $\quad 53$

\section{Introduction}

The measurement of beauty and charm production in ep collisions at HERA is an important testing ground for perturbative Quantum Chromodynamics (pQCD), since the heavy-quark masses provide a hard scale that allows perturbative calculations to be made. At leading order, the dominant process for heavy-quark production at HERA is boson-gluon fusion (BGF). In this process, a virtual photon emitted by the incoming electron interacts with a gluon from the proton forming a heavy quark-antiquark pair. When the negative squared four-momentum of the virtual photon, $Q^{2}$, is large compared to the proton mass, the interaction is referred to as deep inelastic scattering (DIS). For heavy-quark transverse momenta comparable to the quark mass, next-to-leading-order (NLO) QCD calculations based on the dynamical generation of the massive quarks [1-4] are expected to provide reliable predictions.

Beauty and charm production in DIS has been measured using several methods by the H1 [5-16] and ZEUS [17-31] collaborations. All but the two most recent measurements of 
charm production [30, 31] and older data [22] have been combined [32]. Predictions from NLO QCD describe all results reasonably well.

Inclusive jet cross sections in beauty and charm events are used in the analysis presented here to extract the heavy-quark contribution to the proton structure function $F_{2}$ with high precision, and to measure the $b$-quark mass. For this purpose, the long lifetimes of the weakly decaying $b$ and $c$ hadrons, which make the reconstruction of their decay vertices possible, as well as their large masses were exploited. Two discriminating variables, the significance of the reconstructed decay length and the invariant mass of the charged tracks associated with the decay vertex (secondary vertex), were used. This inclusive tagging method leads to a substantial increase in statistics with respect to previous ZEUS measurements.

Differential cross sections as a function of $Q^{2}$, the Bjorken scaling variable, $x$, jet transverse energy, $E_{T}^{\text {jet }}$, and pseudorapidity, $\eta^{\text {jet }}$, were measured. They are compared to a leading-order (LO) plus parton-shower (PS) Monte Carlo prediction and to NLO QCD calculations. The beauty and charm contributions to the proton structure function $F_{2}$, denoted as $F_{2}^{b \bar{b}}$ and $F_{2}^{c \bar{c}}$, respectively, as well as beauty and charm reduced cross sections $\left(\sigma_{\mathrm{r}}^{b \bar{b}}\right.$ and $\sigma_{\mathrm{r}}^{c \bar{c}}$, respectively) were extracted from the double-differential cross section as a function of $Q^{2}$ and $x$. The results are compared to previous measurements and to predictions from perturbative QCD.

The running $\overline{\mathrm{MS}}$ beauty-quark mass, $m_{b}$ at the scale $m_{b}$, denoted $m_{b}\left(m_{b}\right)$, is measured using $\sigma_{\mathrm{r}}^{b \bar{b}}$, following a procedure similar to that used for a recent extraction of the charmquark mass [32]. This represents the first measurement of the $b$-quark mass using HERA or any other hadron collider data.

\section{Experimental set-up}

This analysis was performed with data taken with the ZEUS detector from 2004 to 2007, when HERA collided electrons ${ }^{1}$ with energy $E_{e}=27.5 \mathrm{GeV}$ with protons of energy $920 \mathrm{GeV}$, corresponding to a centre-of-mass energy $\sqrt{s}=318 \mathrm{GeV}$. This data-taking period is denoted as HERA II. The corresponding integrated luminosity is $(354 \pm 7) \mathrm{pb}^{-1}$.

A detailed description of the ZEUS detector can be found elsewhere [33]. A brief outline of the components that are most relevant for this analysis is given below.

In the kinematic range of the analysis, charged particles were tracked in the central tracking detector (CTD) [34-36] and the microvertex detector (MVD) [37]. These components operated in a magnetic field of $1.43 \mathrm{~T}$ provided by a thin superconducting solenoid. The CTD consisted of 72 cylindrical drift-chamber layers, organised in nine superlayers covering the polar-angle ${ }^{2}$ region $15^{\circ}<\theta<164^{\circ}$. The MVD silicon tracker consisted of a barrel (BMVD) and a forward (FMVD) section. The BMVD contained three layers and provided

\footnotetext{
${ }^{1}$ In this paper "electron" is used to denote both electron and positron.

${ }^{2}$ The ZEUS coordinate system is a right-handed Cartesian system, with the $Z$ axis pointing in the nominal proton beam direction, referred to as the "forward direction", and the $X$ axis pointing towards the centre of HERA. The coordinate origin is at the centre of the CTD. The pseudorapidity is defined as $\eta=-\ln \left(\tan \frac{\theta}{2}\right)$, where the polar angle, $\theta$, is measured with respect to the $Z$ axis.
} 
polar-angle coverage for tracks from $30^{\circ}$ to $150^{\circ}$. The four-layer FMVD extended the polarangle coverage in the forward region to $7^{\circ}$. After alignment, the single-hit resolution of the MVD was $24 \mu \mathrm{m}$. The transverse distance of closest approach (DCA) of tracks to the nominal vertex in $X-Y$ was measured to have a resolution, averaged over the azimuthal angle, of $\left(46 \oplus 122 / p_{T}\right) \mu \mathrm{m}$, with $p_{T}$ in GeV. For CTD-MVD tracks that pass through all nine CTD superlayers, the momentum resolution was $\sigma\left(p_{T}\right) / p_{T}=0.0029 p_{T} \oplus 0.0081 \oplus 0.0012 / p_{T}$, with $p_{T}$ in $\mathrm{GeV}$.

The high-resolution uranium-scintillator calorimeter (CAL) [38-41] consisted of three parts: the forward (FCAL), the barrel (BCAL) and the rear (RCAL) calorimeters. Each part was subdivided transversely into towers and longitudinally into one electromagnetic section (EMC) and either one (in RCAL) or two (in BCAL and FCAL) hadronic sections (HAC). The smallest subdivision of the calorimeter was called a cell. The CAL energy resolutions, as measured under test-beam conditions, were $\sigma(E) / E=0.18 / \sqrt{E}$ for electrons and $\sigma(E) / E=0.35 / \sqrt{E}$ for hadrons, with $E$ in $\mathrm{GeV}$.

The luminosity was measured using the Bethe-Heitler reaction $e p \rightarrow e \gamma p$ by a luminosity detector which consisted of independent lead-scintillator calorimeter [42-44] and magnetic spectrometer [45] systems.

\section{Monte Carlo simulations}

To evaluate the detector acceptance and to provide predictions of the signal and background distributions, Monte Carlo (MC) samples of beauty, charm and light-flavour events were generated, corresponding to eighteen, three and one times the integrated luminosity of the data, respectively. The RAPGAP 3.00 MC program [46] in the massive mode $\left(m_{b}=\right.$ $4.75 \mathrm{GeV}, m_{c}=1.5 \mathrm{GeV}$ ) was used to generate the beauty and charm samples, where the CTEQ5L [47] parameterisation for the proton parton density functions (PDFs) was used. In RAPGAP, LO matrix elements are combined with higher-order QCD radiation simulated in the leading-logarithmic approximation. Higher-order QED effects are included through HeraCles 4.6 [48]. Light-flavour MC events were extracted from an inclusive DIS sample generated with DJAngoh 1.6 [49] interfaced to ARIADne 4.12 [50]. The CTEQ5D [47] PDFs were used and quarks were taken to be massless.

Fragmentation and particle decays were simulated using the Jetset/PythiA model [51, 52]. The Bowler parameterisation [53] of the fragmentation function, as implemented in Pyтнia [54] (with $r_{Q}=1$ ), was used for the heavy-flavour samples. The generated events were passed through a full simulation of the ZEUS detector based on GEANT 3.21 [55]. The final MC events were then subjected to the same trigger requirements and processed by the same reconstruction program as the data.

For the acceptance determination, the $E_{T}^{\text {jet }}$ and $\eta^{\text {jet }}$ distributions in the charm MC, as well as the $Q^{2}$ distributions in both the beauty and charm MCs, were reweighted in order to give a good description of the data. The charm branching fractions and fragmentation fractions were adjusted to the world-average values [56-58]. 


\section{Theoretical predictions and uncertainties}

Next-to-leading-order QCD predictions for differential cross sections were obtained from the HvQDis program [59]. The calculations were used to extrapolate the visible cross sections to extract $F_{2}^{b \bar{b}}, F_{2}^{c \bar{c}}, \sigma_{\mathrm{r}}^{b \bar{b}}$ and $\sigma_{\mathrm{r}}^{c \bar{c}}$ (see section 9 ). The calculations are based on the fixed-flavour-number scheme (FFNS) in which only light flavours are present in the proton and heavy quarks are produced in the interaction [60]. Therefore, the 3-flavour (4-flavour) FFNS variant of the ZEUS-S NLO QCD fit [61] was used for the proton PDF for the predictions of the charm (beauty) cross sections. As in the PDF fit, the value of $\alpha_{s}\left(M_{Z}\right)$ was set to 0.118 and the heavy-quark masses (pole masses) were set to $m_{b}=4.75 \mathrm{GeV}$ and $m_{c}=1.5 \mathrm{GeV}$. The renormalisation and factorisation scales, $\mu_{R}$ and $\mu_{F}$, were chosen to be equal and set to $\mu_{R}=\mu_{F}=\sqrt{Q^{2}+4 m_{b(c)}^{2}}$.

The systematic uncertainties on the theoretical predictions with the ZEUS-S PDFs were estimated by varying the quark masses and the renormalisation and factorisation scales. Quark masses of $m_{b}=4.5$ and $5.0 \mathrm{GeV}, m_{c}=1.3$ and $1.7 \mathrm{GeV}$ were used. The scales $\mu_{R}, \mu_{F}$ were varied independently by a factor of two up and down. Additionally, the experimental uncertainties of the data used in the PDF fit were propagated to the predicted cross sections. The total uncertainties were obtained by adding positive and negative changes to the cross sections in quadrature. This results in total uncertainties of 10-20\% for beauty and 10-50\% for charm.

Predictions were also obtained using the 3 - and 4-flavour variants of the ABKM NLO PDFs [62] for the proton. The pole masses of heavy quarks were set to $m_{b}=4.5 \mathrm{GeV}$ and $m_{c}=1.5 \mathrm{GeV}$, both in the PDF fit and in the HvQDis calculation. The values of $\alpha_{s}\left(\mu_{R}\right)$ were provided by LHAPDF $[63,64]$ to ensure that the same function was used as in the PDF fit. The renormalisation and factorisation scales were both set to $\mu_{R}=\mu_{F}=\sqrt{Q^{2}+4 m_{b(c)}^{2}}$.

The NLO QCD predictions are given for parton-level jets. These were reconstructed using the $k_{T}$ clustering algorithm [65] with a radius parameter $R=1.0$ in the longitudinally invariant mode [66]. The $E$-recombination scheme, which produces massive jets whose four-momenta are the sum of the four-momenta of the clustered objects, was used. The parton-level cross sections were corrected for jet hadronisation effects to allow a direct comparison with the measured hadron-level cross sections:

$$
\sigma^{\text {had, NLO }}=C^{\text {had }} \sigma^{\text {parton,NLO }},
$$

where the correction factors, $C^{\text {had }}=1+\Delta^{\text {had }}$, were derived from the RAPGAP MC simulation. The factors $C^{\text {had }}$ are defined as the ratio of the hadron-level jet to the parton-level jet cross sections, and the parton level is defined as the result of the parton-showering stage of the simulation.

Since $C^{\text {had }}$ were derived from an LO plus parton shower MC, but are applied to an NLO prediction, the uncertainty on $C^{\text {had }}$ cannot be estimated in a straightforward way. Within the framework of parton showering, MC subsets with different numbers of radiated partons were investigated using RAPGAP and PYTHIA samples. These studies indicated that different approaches yield variations of $\Delta^{\text {had }}$ of typically a factor of two. Since it is not 
clear if the variations can be interpreted as uncertainties on $C^{\text {had }}$, no such uncertainties were included in the cross-section $\left(F_{2}\right)$ predictions. However, for the extraction of the $b$-quark mass, such a theoretical uncertainty needs to be included.

\section{Data selection}

Events containing a scattered electron were selected online by means of a three-level trigger system $[33,67]$. The trigger [68] did not require the presence of a secondary vertex nor of a jet.

Offline, the scattered electron was reconstructed using an electron finder based on a neural network [69]. The hadronic system was reconstructed from energy-flow objects (EFOs) [70, 71] which combine the information from calorimetry and tracking, corrected for energy loss in the detector material. The kinematic variables used in the cross-section measurements, $Q^{2}$ and $x$, were reconstructed using the double-angle method [72].

The following cuts were applied to select a clean DIS sample:

- the reconstructed scattered electron $[69,73]$ was required to have an energy $E_{e}^{\prime}>$ $10 \mathrm{GeV}$;

- the impact position of the scattered electron on the face of the RCAL had to be outside the region $26 \times 26 \mathrm{~cm}^{2}$ centred on $X=Y=0$;

- the primary vertex had to be within $\pm 30 \mathrm{~cm}$ in $Z$ of the nominal interaction point;

- the photon virtuality, $Q^{2}$, had to be within $5<Q^{2}<1000 \mathrm{GeV}^{2}$;

- $y_{\mathrm{JB}}>0.02$, where $y_{\mathrm{JB}}$ is the inelasticity reconstructed using the Jacquet-Blondel method [74];

- $y_{e}<0.7$, where $y_{e}$ is the inelasticity reconstructed using the electron method [72];

- $44<\left(E-p_{Z}\right)<65 \mathrm{GeV}$, where $\left(E-p_{Z}\right)=\sum_{i}\left(E_{i}-p_{Z, i}\right)$ and $i$ runs over all finalstate particles with energy $E_{i}$ and $Z$-component of momentum $p_{Z, i}$; this selects fully contained neutral-current $e p$ events for which $E-p_{Z}=2 E_{e}$.

Jets were reconstructed from EFOs using the $k_{T}$ clustering algorithm [65] as was described for parton-level jets in section 4. Jets containing the identified scattered electron were not considered further. Events were selected if they contained at least one jet within the pseudorapidity range $-1.6<\eta^{\text {jet }}<2.2$ and with transverse energy, $E_{T}^{\text {jet }}$, of

$$
E_{T}^{\mathrm{jet}}=p_{T}^{\mathrm{jet}} \frac{E^{\mathrm{jet}}}{p^{\mathrm{jet}}}>5(4.2) \mathrm{GeV}
$$

for beauty (charm), where $E^{\text {jet }}, p^{\text {jet }}$ and $p_{T}^{\text {jet }}$ are the jet energy, momentum and transverse momentum. The cut on $E_{T}^{\text {jet }}$ was optimised separately for beauty and charm measurements. For beauty, a cut of $E_{T}^{\text {jet }}>5 \mathrm{GeV}$ ensures a good correlation between momentum and angle 
for reconstructed and hadron-level jets [75]; for charm this cut was $4.2 \mathrm{GeV}$ to reduce the extrapolation uncertainties for the $F_{2}^{c \bar{c}}$ and $\sigma_{\mathrm{r}}^{c \bar{c}}$ measurements at low $Q^{2}$.

In order to reconstruct potential secondary vertices related to $b$ - and $c$-hadron decays, tracks were selected if:

- they had a transverse momentum $p_{T}>0.5 \mathrm{GeV}$;

- the total number of hits ${ }^{3}$ on the track in the MVD was $\geq 4$;

- if the track was inside the CTD acceptance, track recognition in the CTD was required; the percentage of the tracks outside the CTD acceptance, and hence reconstructed using MVD hits only, was $2.5 \%$.

Tracks were associated with the closest jet if they fulfilled the criterion $\Delta R<1$ with $\Delta R=\sqrt{\left(\eta^{\text {trk }}-\eta^{\text {jet }}\right)^{2}+\left(\phi^{\text {trk }}-\phi^{\text {jet }}\right)^{2}}$. If two or more of such tracks were associated with the jet, a candidate vertex was fitted from the selected tracks using a deterministic annealing filter [76-78]. This fit provided the vertex position and its error matrix as well as the invariant mass, $m_{\mathrm{vtx}}$, of the charged tracks associated with the reconstructed vertex. The charged-pion mass was assumed for all tracks when calculating the vertex mass. Vertices with $\chi^{2} /$ ndf $<6$, a distance from the interaction point within $\pm 1 \mathrm{~cm}$ in the $X-Y$ plane, $\pm 30 \mathrm{~cm}$ in the $Z$ direction, and $1<m_{\mathrm{vtx}}<6 \mathrm{GeV}$ were kept for further analysis.

The MC gives a good description of the track efficiencies, except for a small fraction of tracks that are affected by hadronic interactions in the detector material between the interaction point and the CTD. Efficiency corrections for this effect were determined from a study of exclusive $e p \rightarrow e \rho^{0} p$ events [79], using a special track reconstruction. The number of pions from the $\rho^{0}$ decay that were reconstructed in the MVD alone and had no extension in the CTD was measured. The resulting track efficiency correction in the MC was applied by randomly rejecting selected vertex tracks before the vertex fit, with a probability that depends on the track parameters (around $3 \%$ at $\eta=0$ and $p_{T}=1 \mathrm{GeV}$ ).

\section{Extraction of the heavy-flavour cross sections}

Using the secondary-vertex candidates associated with jets, the decay length, $d$, was defined as the vector in $X-Y$ between the secondary vertex and the interaction point ${ }^{4}$ projected onto the jet axis in the $X-Y$ plane. The sign of the decay length was assigned using the axis of the jet to which the vertex was associated; if the decay-length vector was in the same hemisphere as the jet axis, a positive sign was assigned to it, otherwise the sign of the decay length was negative. Negative decay lengths, which originate from secondary vertices reconstructed on the wrong side of the interaction point with respect to the direction of the associated jets, are unphysical and caused by detector resolution effects. A small smearing

\footnotetext{
${ }^{3}$ Each MVD layer provided two coordinate measurements.

${ }^{4}$ In the $X-Y$ plane, the interaction point was defined as the centre of the beam ellipse, determined using the average primary vertex position for groups of a few thousand events, taking into account the difference in angle between the beam direction and the $Z$ direction. The $Z$ coordinate was taken as the $Z$ position of the primary vertex of the event.
} 
correction [79] to the MC decay-length distribution was applied in order to reproduce the data with negative values of decay length.

The beauty and charm content in the selected sample was determined using the shape of the decay-length significance distribution together with the secondary-vertex mass distribution, $m_{\mathrm{vtx}}$. The decay-length significance, $S$, is defined as $d / \delta d$, where $\delta d$ is the uncertainty on $d$. The invariant mass of the tracks fitted to the secondary vertex provides a distinguishing variable for jets from $b$ and $c$ quarks, reflecting the different masses of the $b$ and $c$ hadrons. Figure 1 shows the decay-length significance, $S$, for $E_{T}^{\text {jet }}>4.2 \mathrm{GeV}$ divided into four bins: $1<m_{\mathrm{vtx}}<1.4 \mathrm{GeV}, 1.4<m_{\mathrm{vtx}}<2 \mathrm{GeV}, 2<m_{\mathrm{vtx}}<6 \mathrm{GeV}$ and no restriction on $m_{\mathrm{vtx}}$. The MC simulation provides a good description of the data. The separation into subsamples is described below.

The contents of the negative bins of the significance distribution, $N\left(S^{-}\right)$, were subtracted from the contents of the corresponding positive bins, $N\left(S^{+}\right)$, yielding a subtracted decay-length significance distribution. In this way, the contribution from light-flavour quarks is minimised. An additional advantage of this subtraction is that symmetric systematic effects, which might arise from discrepancies between the data and the MC, are removed. In order to reduce the contamination of tracks originating from the primary vertex, a cut of $|S|>4$ was applied.

To extract the contributions from beauty, charm and light flavours in the data sample, a binned $\chi^{2}$ fit of the subtracted significance distribution in the region $4<|S|<20$ was performed simultaneously for three mass bins [68]: $1<m_{\mathrm{vtx}}<1.4 \mathrm{GeV} ; 1.4<m_{\mathrm{vtx}}<2 \mathrm{GeV}$; $2<m_{\mathrm{vtx}}<6 \mathrm{GeV}$. All MC distributions were normalised to the integrated luminosity of the data before the fit. The overall MC normalisation was constrained by requiring it to be consistent with the normalisation of the data in the significance distribution with $|S|<20$ and $1<m_{\mathrm{vtx}}<6 \mathrm{GeV}$. The fit yielded scaling factors $k_{b}, k_{c}$ and $k_{\mathrm{lf}}$ for the beauty, charm and light-flavour contributions, respectively, to obtain the best description of the data. The correlation coefficients were as follows: $\rho_{b, c}=-0.68(-0.67), \rho_{b, \text { lf }}=0.58(0.57)$ and $\rho_{c, \text { lf }}=-0.98(-0.98)$ for $E_{T}^{\text {jet }}>4.2(5.0) \mathrm{GeV}$. The subtracted and fitted distributions for $E_{T}^{\text {jet }}>4.2 \mathrm{GeV}$ are shown in figure 2. A good agreement between data and MC is observed. The first two mass bins corresponding to the region $1<m_{\mathrm{vtx}}<2 \mathrm{GeV}$ are dominated by charm events. In the third mass bin, beauty events are dominant at high values of significance. The fit procedure was repeated for every bin of a given observable to obtain differential cross sections. For the beauty cross-section extraction, the fit procedure was repeated with the higher cut on $E_{T}^{\mathrm{jet}}, E_{T}^{\mathrm{jet}}>5 \mathrm{GeV}$.

Control distributions of $E_{T}^{\text {jet }}, \eta^{\text {jet }}, \log _{10} Q^{2}$ and $\log _{10} x$ are shown in figure 3 after beauty enrichment cuts $\left(2<m_{\mathrm{vtx}}<6 \mathrm{GeV}\right.$ and $\left.|S|>8\right)$ for $E_{T}^{\text {jet }}>5.0 \mathrm{GeV}$ and in figure 4 after charm enrichment cuts $\left(1<m_{\mathrm{vtx}}<2 \mathrm{GeV}\right.$ and $\left.|S|>4\right)$ for $E_{T}^{\text {jet }}>4.2 \mathrm{GeV}$. All data distributions are reasonably well described by the MC.

The differential cross sections for jet production in beauty or charm events, $q=b, c$, corrected to QED Born level, in a bin $i$ of a given observable, $Y$, are given by:

$$
\frac{\mathrm{d} \sigma_{q}^{\mathrm{jet}}}{\mathrm{d} Y_{i}}=k_{q}\left(Y_{i}\right) \frac{N_{q}^{\mathrm{had}, \mathrm{MC}}\left(Y_{i}\right)}{\mathcal{L} \cdot \Delta Y_{i}} \frac{1}{C^{\mathrm{rad}}},
$$


where $\Delta Y_{i}$ is the width of the bin, $k_{q}$ denotes the scaling factor obtained from the fit, $N_{q}^{\text {had,MC }}$ is the number of generated jets in beauty or charm events at the MC hadron level, $C^{\text {rad }}$ is the QED radiative correction and $\mathcal{L}$ is the corresponding integrated luminosity.

Hadron-level jets were obtained by running the $k_{T}$ clustering algorithm on all stable final-state particles, in the same mode as for the data. Weakly decaying $b$ and $c$ hadrons were treated as stable particles and were decayed only after the application of the jet algorithm.

The predictions from the HVQDIS program are given at the QED Born level with a running coupling, $\alpha_{\mathrm{em}}$. Hence, a correction of the measured cross sections for QED radiative effects is necessary in order to be able to compare them directly to the HVQDIS predictions. The corrections were obtained using the RAPGAP Monte Carlo as $C^{\mathrm{rad}}=\sigma_{\mathrm{rad}} / \sigma_{\mathrm{Born}}$, where $\sigma_{\text {rad }}$ is the cross section with full QED corrections, as used in the standard MC samples, and $\sigma_{\text {Born }}$ was obtained with the QED corrections turned off but keeping $\alpha_{\text {em }}$ running. Both cross sections, $\sigma_{\text {rad }}$ and $\sigma_{\text {Born }}$, were obtained at the hadron level.

\section{Systematic uncertainties}

The systematic uncertainties were evaluated by varying the analysis procedure or by changing the selection cuts and repeating the extraction of the cross section. The following sources of experimental systematic uncertainties were identified [68, 79]; the uncertainties on the integrated cross sections determined for each source are summarised in table 1 to indicate the sizes of the different effects:

$\delta_{1}$ DIS selection - the cuts for DIS event selection were varied in both data and MC. The cut on the scattered electron energy was varied between $9<E_{e}^{\prime}<11 \mathrm{GeV}\left(\delta_{1}^{E_{e}}\right)$, the cut on the inelasticity was varied between $0.01<y_{\mathrm{JB}}<0.03\left(\delta_{1}^{y}\right)$, and the lower cut on $E-p_{Z}$ was changed by $\pm 2 \mathrm{GeV}\left(\delta_{1}^{E-p_{Z}}\right)$;

$\delta_{2}$ trigger efficiency - the uncertainty on the trigger efficiency was evaluated by comparing events taken with independent triggers;

$\delta_{3}$ tracking efficiency correction - the size of the correction was varied by its estimated uncertainty of $\pm 50 \%$;

$\delta_{4}$ decay-length smearing - the fraction of secondary vertices for which the decay length was smeared was varied separately in the core $\left(\delta_{4}^{\text {core }}\right)$ and the tails $\left(\delta_{4}^{\text {tail }}\right)$ of the distribution such that the agreement between data and MC remained reasonable;

$\delta_{5}$ signal extraction procedure - the systematic uncertainty on the signal extraction procedure was estimated by changing the lower $|S|$ cut from $|S|>4$ to $|S|>3$ and $|S|>5$;

$\delta_{6}$ jet energy scale - the calorimetric part of the transverse jet energy in the MC was varied by its estimated uncertainty of $\pm 3 \%$;

$\delta_{7}$ electron energy scale - the reconstructed energy of the scattered electron was varied in the MC by its estimated uncertainty of $\pm 2 \%$; 
$\delta_{8}$ MC model dependence - the $Q^{2}\left(\delta_{8}^{Q^{2}}\right), \eta^{\text {jet }}\left(\delta_{8}^{\eta^{\text {jet }}}\right)$ and $E_{T}^{\text {jet }}\left(\delta_{8}^{E_{T}^{\text {jet }}}\right)$ reweighting corrections in the charm $\mathrm{MC}$ were varied in a range for which the description of data by $\mathrm{MC}$ remained reasonable. The same relative variations were applied to the beauty MC;

$\delta_{9}$ light-flavour background - the light-flavour contribution to the subtracted significance distribution includes a contribution from long-lifetime strange-hadron decays. To estimate the uncertainty due to modelling of this effect, the MC light-flavour distribution of $N\left(S^{+}\right)-N\left(S^{-}\right)$was scaled by $\pm 30 \%$ [14] and the fit was repeated;

$\delta_{10}$ charm fragmentation function - to estimate the sensitivity to the charm fragmentation function, it was changed in the MC from the Bowler to the Peterson [80] parameterisation with $\epsilon=0.062$ [81];

$\delta_{11}$ beauty fragmentation function - to estimate the sensitivity to the beauty fragmentation function, it was changed in the MC from the Bowler to the Peterson parameterisation with $\epsilon=0.0041$ [82];

$\delta_{12}$ charm branching fractions $\left(\delta_{12}^{\mathrm{BR}}\right)$ and fragmentation fractions $\left(\delta_{12}^{\mathrm{frag}}\right)$ - these were varied within the uncertainties of the world-average values [56-58];

$\delta_{13}$ luminosity measurement - a $1.9 \%$ overall normalisation uncertainty was associated with the luminosity measurement.

To evaluate the total systematic uncertainty on the integrated cross sections, the contributions from the different systematic uncertainties were added in quadrature, separately for the negative and the positive variations. The same procedure was applied to each bin for the differential cross sections. However, the luminosity measurement uncertainty was not included. In the case of beauty, the dominant effects arise from the uncertainties on the track-finding inefficiencies, the beauty fragmentation function and MC modelling. For charm, the uncertainties on the branching fractions, the light-flavour asymmetry as well as on the MC modelling contribute most to the total systematic uncertainty.

\section{Cross sections}

Cross sections for inclusive jet production in beauty (charm) events were measured in the range $E_{T}^{\text {jet }}>5(4.2) \mathrm{GeV},-1.6<\eta^{\text {jet }}<2.2$ for DIS events with $5<Q^{2}<1000 \mathrm{GeV}^{2}$ and $0.02<y<0.7$, where the jets are defined as in section 6 . The single-differential cross sections for jet production in beauty and charm events were measured as a function of $E_{T}^{\text {jet }}, \eta^{\text {jet }}, Q^{2}$ and $x$. The results of the measured cross sections are given in tables $2-5$ and shown in figures 5-8. The measurements are compared to the HVQDIS NLO QCD predictions obtained using ZEUS-S and ABKM as proton PDFs, and to the RAPGAP predictions scaled by a factor of 1.49 for beauty and 1.40 for charm. The scale factors correspond to the ratio of the measured integrated visible cross section to the RAPGAP prediction. The shapes of all measured beauty cross sections are reasonably well described by HVQDIS and the RAPGAP MC. RAPGAP provides a worse description of the shape of 
the charm cross sections than HvQDIS. ${ }^{5}$ For charm, the data are typically $20-30 \%$ above the HVQDIS NLO prediction, but in reasonable agreement within uncertainties. Differences between the NLO predictions using the different proton PDFs are mostly very small.

Double-differential cross sections as a function of $x$ for different ranges of $Q^{2}$ for inclusive jet production in beauty and charm events are listed in tables 6 and 7, respectively.

\section{Extraction of $F_{2}^{q \bar{q}}$ and $\sigma_{\mathrm{r}}^{q \bar{q}}$}

The heavy-quark contribution to the proton structure function $F_{2}, F_{2}^{q \bar{q}}$ with $q=b, c$, can be defined in terms of the inclusive heavy flavour double-differential cross section as a function of $x$ and $Q^{2}$,

$$
\frac{\mathrm{d}^{2} \sigma_{q \bar{q}}}{\mathrm{~d} x \mathrm{~d} Q^{2}}=\frac{2 \pi \alpha_{\mathrm{em}}^{2}}{x Q^{4}}\left\{\left[1+\left(1-y^{2}\right)\right] F_{2}^{q \bar{q}}\left(x, Q^{2}\right)-y^{2} F_{L}^{q \bar{q}}\left(x, Q^{2}\right)\right\},
$$

where $F_{L}^{q \bar{q}}$ is the heavy-quark contribution to the structure function $F_{L}$.

To extract $F_{2}^{q \bar{q}}$ from the visible jet production cross sections in heavy-quark events, measured in bins of $x$ and $Q^{2}$, an extrapolation from the measured range in $E_{T}^{\text {jet }}$ and $\eta^{\text {jet }}$ to the full kinematic phase space was performed. This implicitly takes into account the jet multiplicity. The measured values of $F_{2}^{q \bar{q}}$ at a reference point in the $x-Q^{2}$ plane were calculated using

$$
F_{2}^{q \bar{q}}\left(x, Q^{2}\right)=\frac{\mathrm{d}^{2} \sigma_{q}^{\mathrm{jet}} / \mathrm{d} x \mathrm{~d} Q^{2}}{\mathrm{~d}^{2} \sigma_{q}^{\text {had,NLO }} / \mathrm{d} x \mathrm{~d} Q^{2}} F_{2}^{q \bar{q}, \mathrm{NLO}}\left(x, Q^{2}\right),
$$

where $\mathrm{d}^{2} \sigma_{q}^{\text {jet }} / \mathrm{d} x \mathrm{~d} Q^{2}$ is determined in analogy to eq. (6.1), and $F_{2}^{q \bar{q}, \mathrm{NLO}}$ and $\mathrm{d}^{2} \sigma_{q}^{\text {had,NLO }} / \mathrm{d} x \mathrm{~d} Q^{2}$ were calculated at NLO in the FFNS using the HVQDIS program with the factor $C^{\text {had }}$ applied as in eq. (4.1). The proton PDFs were obtained from the FFNS variant of the HERAPDF 1.0 NLO QCD fit [32]. This PDF was used in order to be consistent with the HERA combined results [32]. The strong coupling constant $\alpha_{s}\left(M_{Z}\right)$ was set to 0.105 as in the PDF fit. Other settings were as described in section 4 for the ZEUS-S variant. As discussed in section $6, \mathrm{~d}^{2} \sigma_{q}^{\text {jet }} / \mathrm{d} x \mathrm{~d} Q^{2}$ was multiplied by $1 / C_{q}^{\mathrm{rad}}$, hence $F_{2}^{q \bar{q}}$ is given at QED Born level, consistent with the usual convention. The procedure of eq. (9.1) also corrects for the $F_{L}^{q \bar{q}}$ contribution to the cross section. This assumes that the calculation correctly predicts the ratio $F_{L}^{q \bar{q}} / F_{2}^{q \bar{q}}$.

The extrapolation factors for beauty due to cuts on $E_{T}^{\text {jet }}$ and $\eta^{\text {jet }}$ typically range from 1.3 to 1.0 , decreasing with increasing $Q^{2}$. The factor is up to 1.7 at high values of $x$. For charm, the extrapolation factors are typically about 4 in the region $5<Q^{2}<20 \mathrm{GeV}^{2}$ and about 2 in the region $20<Q^{2}<60 \mathrm{GeV}^{2}$. The uncertainty on the extrapolation from the measured range to the full kinematic phase space was estimated by varying the parameters of the calculation for the extrapolation factors and adding the resulting uncertainties in quadrature. For charm, the same variations were performed as for the HERA combined results [32]: the charm mass was varied by $\pm 0.15 \mathrm{GeV}$; the strong

\footnotetext{
${ }^{5}$ For the acceptance corrections, the Monte Carlo was reweighted as discussed in section 3.
} 
coupling constant $\alpha_{s}\left(M_{Z}\right)$ was changed by \pm 0.002 ; renormalisation and factorisation scales were multiplied simultaneously by 0.5 or 2 . Uncertainties resulting from the proton PDF uncertainty are small ([83], see table 5 in ref. [32] and column "d30" in https://www.desy.de/h1zeus/combined_results/heavy_flavours/Comb_Input_Tables/d12172.charm-ep.dat) and were neglected. For beauty, the same variations of $\alpha_{s}$ and scales were made and the beauty mass was varied by $\pm 0.25 \mathrm{GeV}$. For each bin, a reference point in $x$ and $Q^{2}$ was defined (see table 8 ) to calculate the structure function.

In addition, beauty and charm reduced cross sections were determined. They are defined as

$$
\sigma_{\mathrm{r}}^{q \bar{q}}=\frac{\mathrm{d}^{2} \sigma_{q \bar{q}}}{\mathrm{~d} x \mathrm{~d} Q^{2}} \cdot \frac{x Q^{4}}{2 \pi \alpha_{\mathrm{em}}^{2}\left[1+\left(1-y^{2}\right)\right]}=F_{2}^{q \bar{q}}\left(x, Q^{2}\right)-\frac{y^{2}}{1+\left(1-y^{2}\right)} F_{L}^{q \bar{q}}\left(x, Q^{2}\right),
$$

and are extracted in analogy to $F_{2}^{q \bar{q}}$ as described above except that no assumption on $F_{L}^{q \bar{q}}$ is required.

The extracted values of $F_{2}^{b \bar{b}}$ and $F_{2}^{c \bar{c}}$ are given in tables 8 and 9 , respectively, while $\sigma_{\mathrm{r}}^{b \bar{b}}$ and $\sigma_{\mathrm{r}}^{c \bar{c}}$ are shown in tables 10 and 11. The total uncertainties of the measurements were calculated from the statistical and systematic uncertainties of the measured cross sections (tables 6, 7, 12-15) and of the extrapolation uncertainty (tables 16-19), added in quadrature.

The structure function $F_{2}^{c \bar{c}}$ is shown in figure 9 as a function of $x$ for different values of $Q^{2}$. The measurements are compared to the NLO QCD HERAPDF 1.5 [84] predictions, the most recent official release of the HERAPDF, based on the RT [85] general-mass variableflavour-number scheme (GMVFNS). The predictions are consistent with the measurements.

In figure 10, the measured $\sigma_{\mathrm{r}}^{c \bar{c}}$ values are compared to the HERA combined results [32] as well as to the two recent results from ZEUS [30,31] which are not yet included in the combination. For the comparison, some of the measured values of this analysis were swum in $Q^{2}$ and $x$ using HvQDIs. This measurement is competitive, especially at high $Q^{2}$, where the extrapolation uncertainty is low, and is in agreement with the HERA combined measurements.

The structure function $F_{2}^{b \bar{b}}$ is shown in figure 11 as a function of $x$ for different values of $Q^{2}$. The measurements are compared to HERAPDF 1.5 GMVFNS predictions. The increase in the uncertainty on the prediction around $Q^{2}=m_{b}^{2}$ is a feature of the GMVFNS scheme used. The predictions are consistent with the measurements.

The $F_{2}^{b \bar{b}}$ measurement is also shown as a function of $Q^{2}$ for fixed $x$ in figure 12 , and is compared to previous ZEUS and H1 measurements. Again, HvQDIS was used to swim the measured values in $Q^{2}$ and $x$ to match the previous measurements. In a wide range of $Q^{2}$, this measurement represents the most precise determination of $F_{2}^{b \bar{b}}$ at HERA. It is in good agreement with the previous ZEUS analyses and H1 measurements. Several NLO and NNLO QCD predictions based on the fixed- or variable-flavour-number schemes [62, 84-90] are also compared to the measurements. Predictions from different theoretical approaches agree well with each other. All predictions provide a reasonable description of the data. 


\section{Measurement of the running beauty-quark mass}

The reduced beauty cross sections, $\sigma_{\mathrm{r}}^{b \bar{b}}$, (figure 13 and table 10) together with inclusive DIS data were used to determine the beauty-quark mass, in a simultaneous fit of the mass and the parton densities.

The measurement procedure follows closely the method presented in a recent H1-ZEUS publication [32], where the running charm-quark mass in the $\overline{\mathrm{MS}}$ scheme was extracted using a simultaneous QCD fit of the combined HERA I inclusive DIS data [91] and the HERA combined charm DIS data [32]. This approach was also used and extended by a similar independent analysis [92], and was preceded by a similar analysis of a partial charm data set [93].

The fit for the running beauty-quark mass was performed within the HERAFitter [94] framework choosing the ABM implementation of the fixed-flavour-number scheme at nextto-leading order $[3,4,62,95,96]$. The OPENQCDRAD [97] option in HERAFitter was used in the $\overline{\mathrm{MS}}$ running-mass mode. The fit was applied to the beauty data listed in table 10 and to the same inclusive DIS data [91] as in the charm-quark mass fit [32]. A fit to the inclusive data only shows a very weak dependence on $m_{b}$. In order to avoid technical complications, no charm data were included in the simultaneous fit and only $m_{b}$ was extracted.

The PDFs resulting from the simultaneous fit changed only marginally with respect to the nominal PDFs obtained from the fit to the inclusive DIS data only. The $\chi^{2}$ of the QCD fit, including the beauty data, shows a clear dependence on the beauty-quark mass, $m_{b}$, as can be seen in figure 14. The total $\chi^{2}$ for the best fit is 587 for 596 degrees of freedom, and the partial contribution from the beauty data is 11.4 for 17 points. The beauty-quark mass and its uncertainty are determined from a parabolic parameterisation. The best fit yields

$$
\left.\left.m_{b}\left(m_{b}\right)=4.07 \pm 0.14(\text { fit })_{-0.07}^{+0.01} \text { (mod. }\right)_{-0.00}^{+0.05} \text { (param. }\right)_{-0.05}^{+0.08} \text { (theo.) } \mathrm{GeV}
$$

for the $\overline{\mathrm{MS}}$ running beauty-quark mass at NLO. The fit uncertainty ${ }^{6}$ (fit) is determined from $\Delta \chi^{2}=1$. It contains the experimental uncertainties, the extrapolation uncertainties, the uncertainties of the standard PDF parameterisation, as well as an estimate of the uncertainty on the hadronisation corrections, as detailed below. In addition, the result has uncertainties attributed to the choices of some extra model parameters (mod.), some additional variations of the PDF parameterisation (param.) and uncertainties on the perturbative QCD parameters (theo.). Details of the uncertainty evaluation include:

Fit uncertainty: For the beauty data, all uncertainties from tables 12, 13 (experimental) and 18 (extrapolation), and the statistical uncertainty, as summarised in table 10, were accounted for in the fit. Following the discussion in section 4, an uncertainty of $100 \%$ on $\Delta^{\text {had }}=C^{\text {had }}-1$ (table 6 ) was introduced as an additional uncorrelated uncertainty. The uncertainties arising from the default PDF parameterisation [32], including the so-called "flexible" gluon parameterisation, are implicitly part of the fit uncertainty.

\footnotetext{
${ }^{6}$ For the charm-quark mass fit [32] this uncertainty was denoted "exp".
} 
The statistical uncertainties and the uncertainties $\delta_{1}, \delta_{2}, \delta_{4}^{\text {core }}, \delta_{5}$ and $\delta_{12}$ from tables 12 and 13 were treated as uncorrelated, while all other uncertainties, including those from luminosity and from table 18, were treated as point-to-point correlated. The "multiplicative" uncertainty option [94] from HERAFitter was used. In the case of asymmetric uncertainties, the larger was used in both directions. The uncertainties of the inclusive data were used as published. Since the inclusive data were taken during the HERA I phase and the beauty data during the HERA II phase, the two sets of data were treated as uncorrelated.

Model uncertainty: The model choices include an assumption on the strangeness fraction, $f_{s}$, the minimum $Q^{2}$ used in the data selection, $Q_{\min }^{2}$, and $Q_{0}^{2}$, the starting value for the $\mathrm{QCD}$ evolution. These were treated exactly as in the charm-quark mass fit [32]. Table 20 lists the choices and variations and their individual contributions to the model uncertainty attributed to the model choices.

Another source of uncertainty is that the $b$-quark mass was used earlier to extrapolate the measured visible cross sections to the reduced cross sections. The corresponding uncertainty is parameterised in table 18 and used in the fit, but the correlation of this uncertainty with the mass used in the QCD fit is lost. Since the HvQDIS [59] program used for the extrapolation uses the pole-mass scheme, and no differential calculations are available in the running-mass scheme, no fully consistent treatment of this correlation is possible. However, the equivalent uncertainty when using the polemass scheme can be consistently estimated. For this purpose, the fit was repeated with the pole-mass option of OPENQCDRAD, which was checked to yield results consistent with the HVQDIS predictions for $\sigma_{\mathrm{r}}^{b \bar{b}}$.

The result, $m_{b}$ (pole) $=4.35 \pm 0.14$ (fit) $\mathrm{GeV}$, has a fit uncertainty which is the same as the fit uncertainty for the running-mass fit. However, since the pole-mass definition includes an unavoidable additional theoretical uncertainty due to a nonperturbative contribution, no attempt to extract a pole-mass measurement with full systematic uncertainties was made. To recover the correlation between the extrapolation and the mass fit, the extrapolated cross sections were iteratively modified using the predictions from the mass values obtained in each fit. This removes the uncertainty on $m_{b}$ in the extrapolation and takes the full correlations into account. The resulting mass $m_{b}$ (pole) $=4.28 \pm 0.13$ (fit) $\mathrm{GeV}$ is slightly lower. The difference between the results from the two procedures $\left(\delta m_{\text {ext }}=-0.07 \mathrm{GeV}\right)$ was treated as an additional model uncertainty.

PDF parameterisation uncertainty: The parameterisation of the PDFs is chosen as for the charm-quark mass fit [32], including the "flexible" parameterisation of the gluon distribution. An additional uncertainty is estimated by freeing three extra PDF parameters $D_{u_{v}}, D_{\bar{D}}$ and $D_{\bar{U}}$ in the fit which allow for small shape variations in the $u_{v}, \bar{U}$ and $\bar{D}$ parton distributions [32]. The change in $m_{b}$ due to leaving the parameters free is given as the systematic uncertainty in table 20 . 
Perturbative scheme and related theory uncertainty: The parameters used for the perturbative part of the QCD calculations also introduce uncertainties; the effects are listed in table 20. As in previous analyses [32, 92, 93], the $\overline{\mathrm{MS}}$ running-mass scheme [98-100] was chosen for all calculations of the reduced cross sections and the fit because it shows better perturbative convergence behaviour than the pole-mass scheme. In order to allow the low- $Q^{2}$ points of the inclusive DIS measurement to be included without the need of additional charm-quark mass corrections, the number of active flavours (NF) was set to three, i.e. the charm contribution was also treated in the fixed-flavour-number scheme. Accordingly, the strong coupling constant was set to $\alpha_{s}\left(M_{Z}\right)^{\mathrm{NF}=3}=0.105 \pm 0.002$, corresponding to $\alpha_{s}\left(M_{Z}\right)^{\mathrm{NF}=5}=0.116 \pm 0.002$.

The theoretical prediction of the charm contribution to the inclusive DIS data is obtained using the running charm-quark mass obtained from the fit to the combined HERA charm data [32], i.e. $m_{c}\left(m_{c}\right)=(1.26 \pm 0.06) \mathrm{GeV}$. It was checked that, as expected, using this mass together with the central PDF from the $m_{b}$ fit, a good description of the combined HERA charm data [32] was obtained. Thus, the charm contribution to the inclusive data should be well described.

The renormalisation and factorisation scales were set to $\mu=\mu_{R}=\mu_{F}=\sqrt{Q^{2}+4 m^{2}}$ with $m=0, m_{c}, m_{b}$ for the light quark, charm, and beauty contributions, respectively, and varied simultaneously by a factor two as in previous analyses [92, 93].

The measured beauty-quark mass is in very good agreement with the world average $m_{b}\left(m_{b}\right)=(4.18 \pm 0.03) \mathrm{GeV}$ [101]. The resulting predictions for the beauty cross sections are shown together with the data in figure 13. Figure 13 also shows the change in the predictions resulting from the fit when different $m_{b}$ values are assumed. The largest sensitivity to $m_{b}$ arises from the low- $Q^{2}$ region, while at high $Q^{2}$ the impact of the beauty-quark mass is small.

\section{Conclusions}

Inclusive jet production cross sections in events containing beauty or charm quarks have been measured in DIS at HERA, exploiting the long lifetimes and large masses of $b$ and $c$ hadrons. In contrast to previous analyses at ZEUS, the measurement was not restricted to any particular final state. This resulted in substantially increased statistics.

Differential cross sections as functions of $E_{T}^{\text {jet }}, \eta^{\text {jet }}, Q^{2}$ and $x$ were determined. Nextto-leading-order QCD predictions calculated using the HVQDIS program using two different sets of proton PDFs are consistent with the measurements.

The heavy-quark contributions to the proton structure function $F_{2}$ as well as beauty and charm reduced cross sections were extracted from the double-differential cross sections as a function of $x$ and $Q^{2}$. The $F_{2}^{b \bar{b}}, F_{2}^{c \bar{c}}$ and $\sigma_{\mathrm{r}}^{b \bar{b}}, \sigma_{\mathrm{r}}^{c \bar{c}}$ values are in agreement with previous measurements at HERA. The results were also compared to several NLO and NNLO QCD calculations, which provide a good description of the data. The precision of the $F_{2}^{c \bar{c}}$ measurement is competitive with other analyses. For a wide range of $Q^{2}$, the $F_{2}^{b \bar{b}}$ measurement represents the most precise determination of $F_{2}^{b \bar{b}}$. 
The running beauty-quark mass in the $\overline{\mathrm{MS}}$ scheme was determined from an NLO QCD fit in the fixed-flavour-number scheme to the $\sigma_{\mathrm{r}}^{b \bar{b}}$ cross sections from this analysis and to HERA I inclusive DIS data:

$$
m_{b}\left(m_{b}\right)=4.07 \pm 0.14(\text { fit })_{-0.07}^{+0.01}(\bmod .)_{-0.00}^{+0.05} \text { (param.) }{ }_{-0.05}^{+0.08} \text { (theo.) } \mathrm{GeV}
$$

This value agrees well with the world average.

\section{Acknowledgments}

We appreciate the contributions to the construction, maintenance and operation of the ZEUS detector of many people who are not listed as authors. The HERA machine group and the DESY computing staff are especially acknowledged for their success in providing excellent operation of the collider and the data-analysis environment. We thank the DESY directorate for their strong support and encouragement. It is a pleasure to thank the ABKM, CTEQ, JR and MSTW groups that provided the predictions for $F_{2}^{b \bar{b}}$ shown in figure 12. We gratefully acknowledge the advice from S. Alekhin and R. Plačakytè concerning the appropriate usage of OPENQCDRAD and HERAFitter.
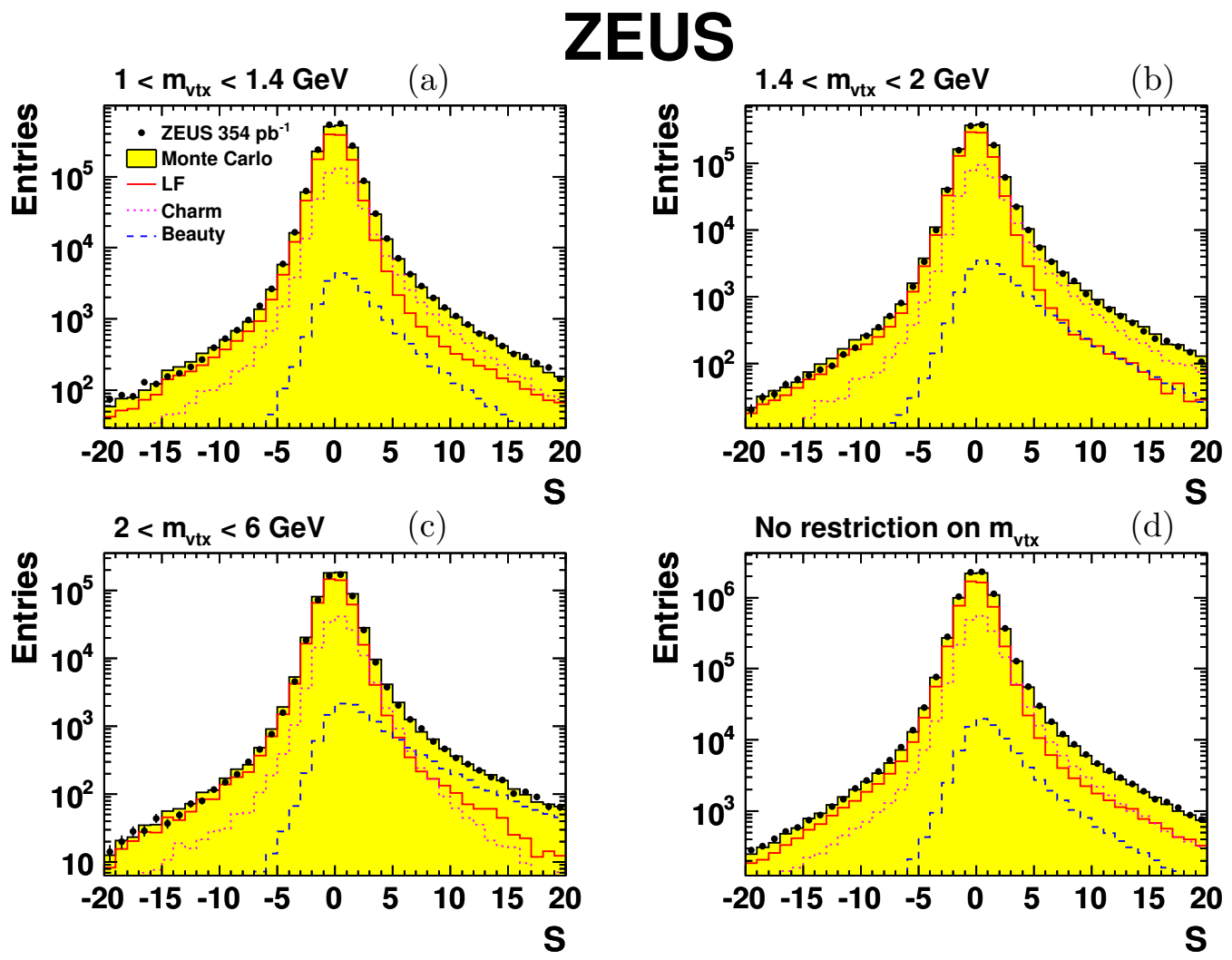

Figure 1. Distributions of the decay-length significance, $S$, for (a) $1<m_{\mathrm{vtx}}<1.4 \mathrm{GeV}$, (b) $1.4<m_{\mathrm{vtx}}<2 \mathrm{GeV}$, (c) $2<m_{\mathrm{vtx}}<6 \mathrm{GeV}$ and (d) no restriction on $m_{\mathrm{vtx}}$. The data are compared to the sum of all MC distributions as well as the individual contributions from the beauty, charm and light-flavour (LF) MC subsamples. All samples were normalised according to the scaling factors obtained from the fit (see text). 


\section{ZEUS}
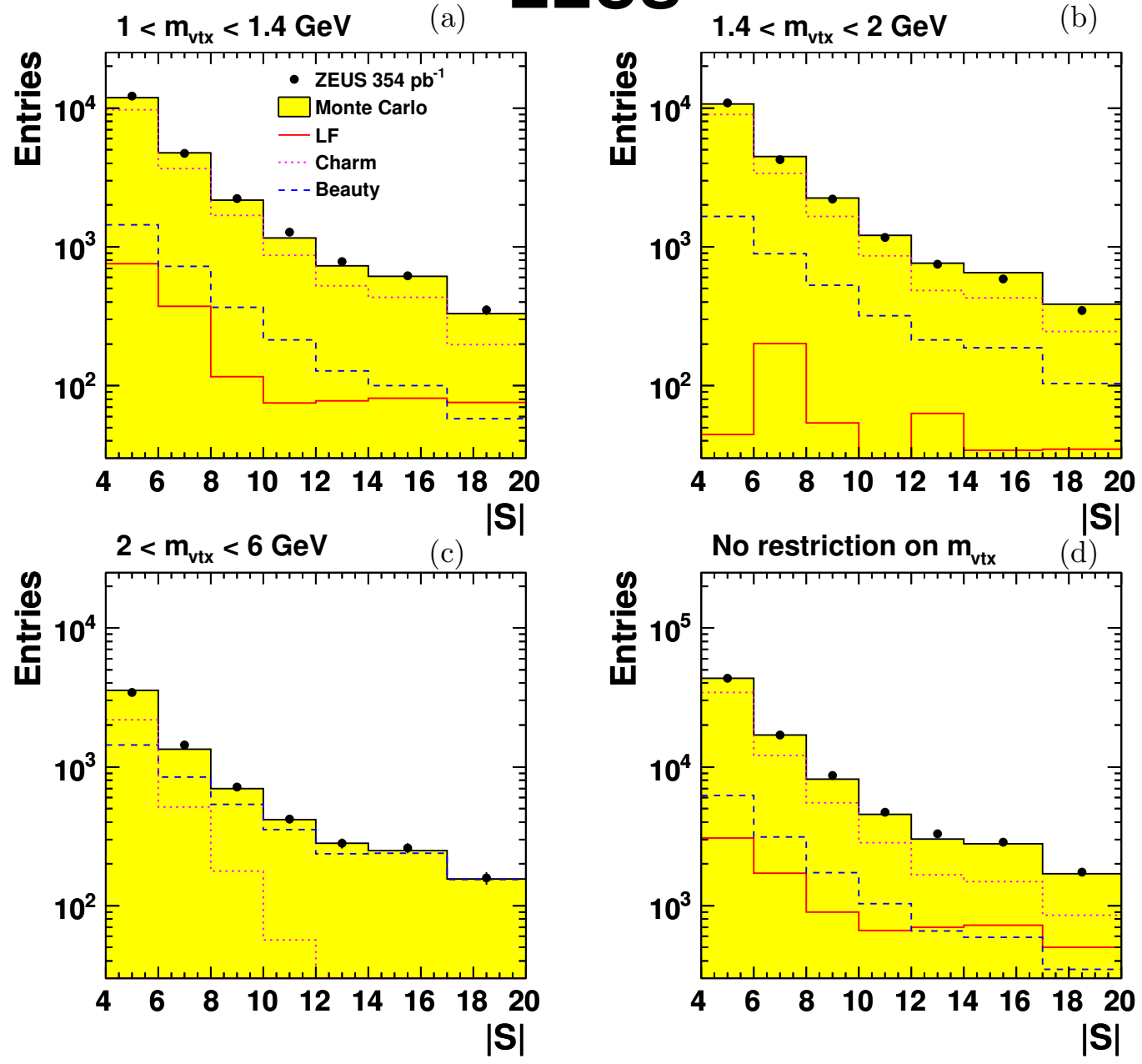

Figure 2. Distribution of the subtracted decay-length significance in four ranges of $m_{\mathrm{vtx}}$. For more details, see the caption of figure 1. 


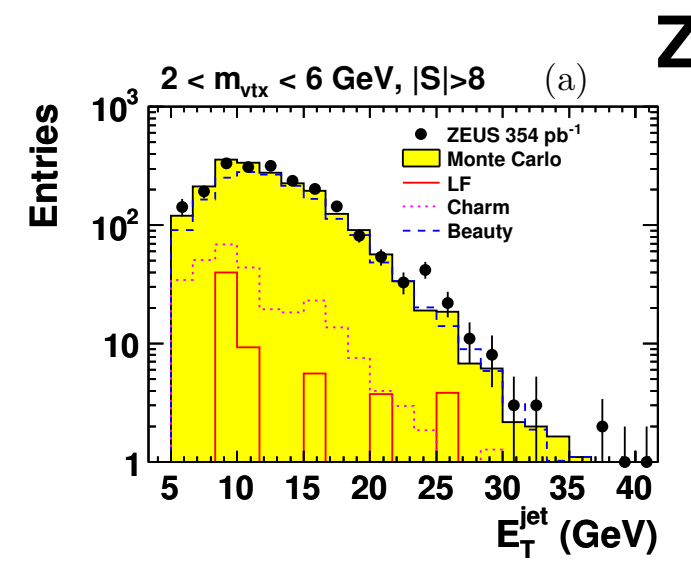

ZEUS

(b)
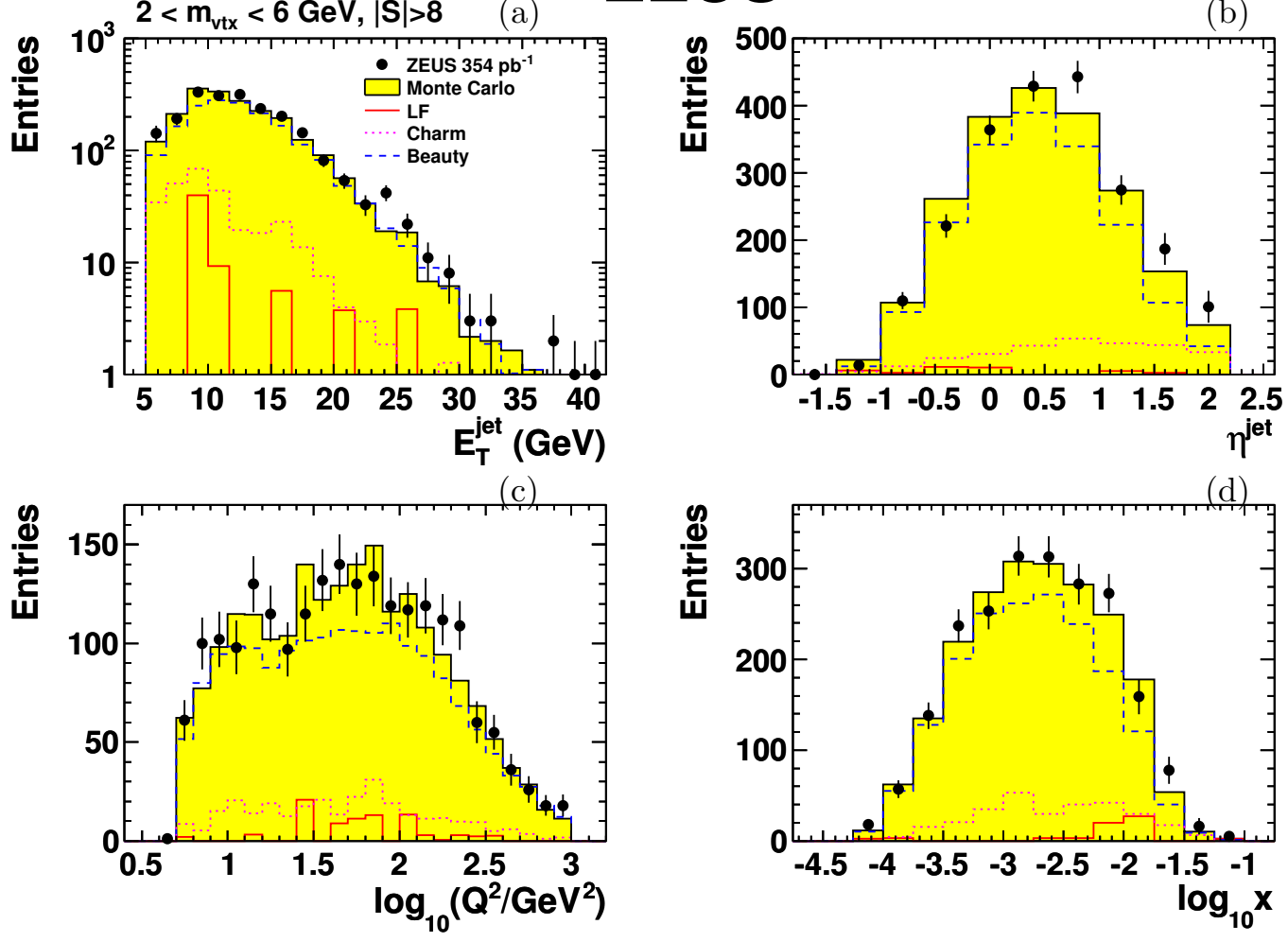

Figure 3. Distributions of (a) $E_{T}^{\text {jet }}$, (b) $\eta^{\text {jet }}$, (c) $\log _{10} Q^{2}$ and (d) $\log _{10} x$ of the selected secondary vertices for a beauty-enriched subsample with $2<m_{\mathrm{vtx}}<6 \mathrm{GeV}$ and $|S|>8$. For more details, see the caption of figure 1. 


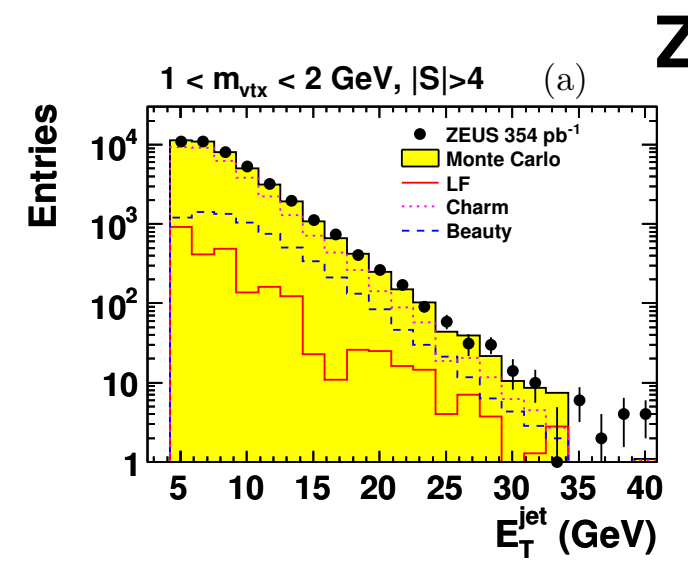

ZEUS
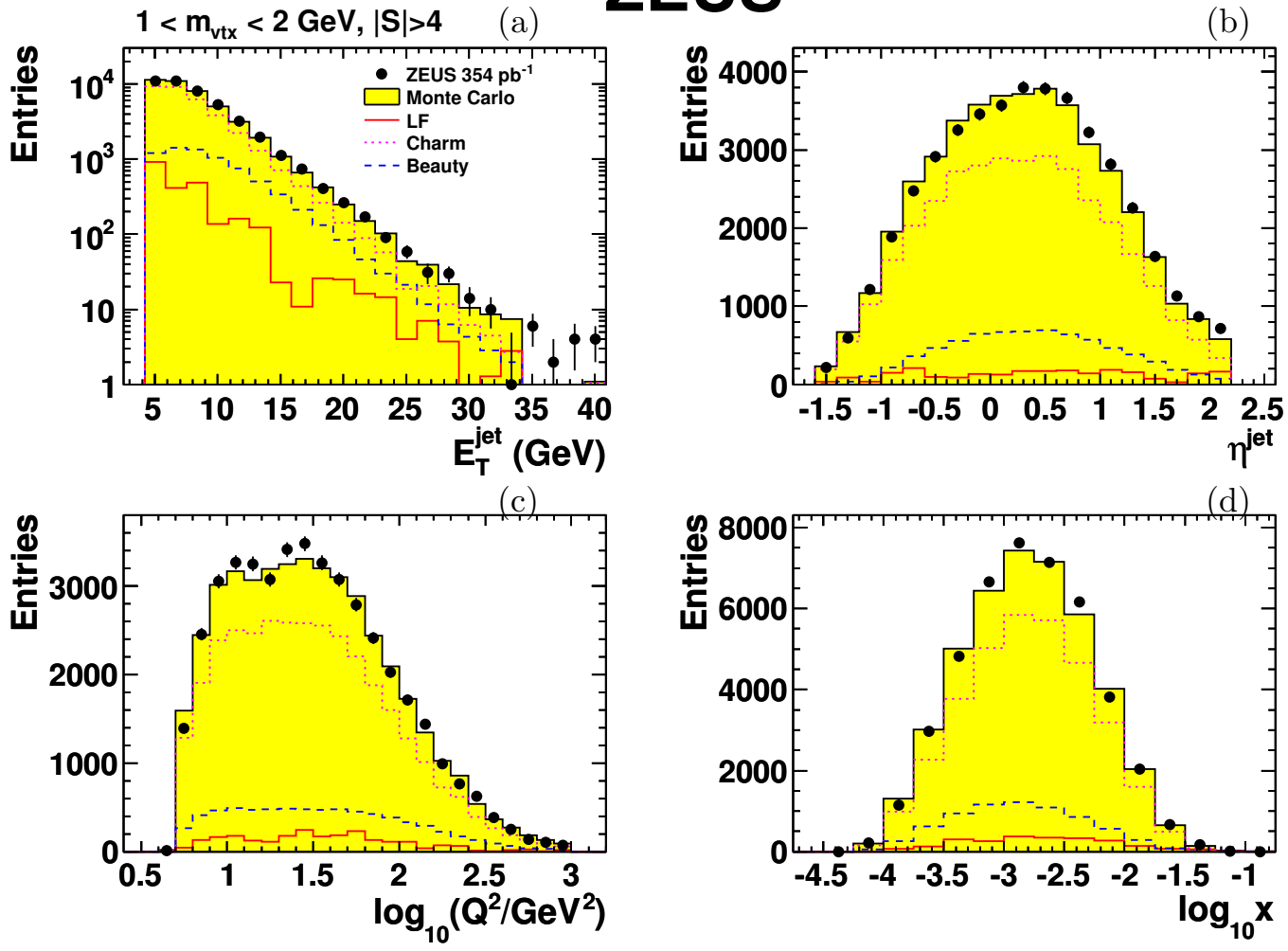

Figure 4. Distributions of (a) $E_{T}^{\text {jet }}$, (b) $\eta^{\text {jet }}$, (c) $\log _{10} Q^{2}$ and (d) $\log _{10} x$ of the selected secondary vertices for a charm-enriched subsample with $1<m_{\mathrm{vtx}}<2 \mathrm{GeV}$ and $|S|>4$. For more details, see the caption of figure 1 . 
ZEUS

(a)
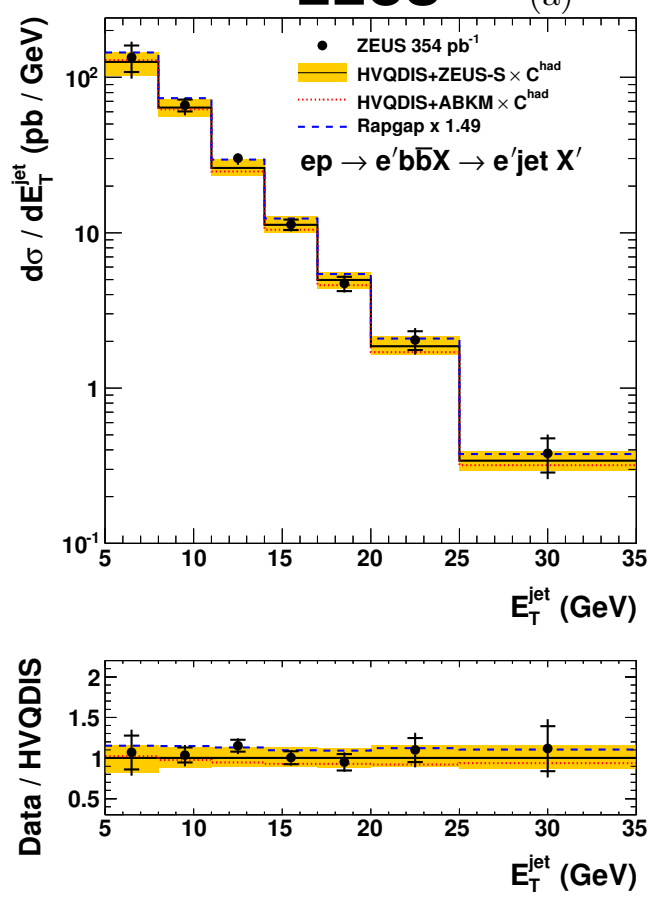

ZEUS
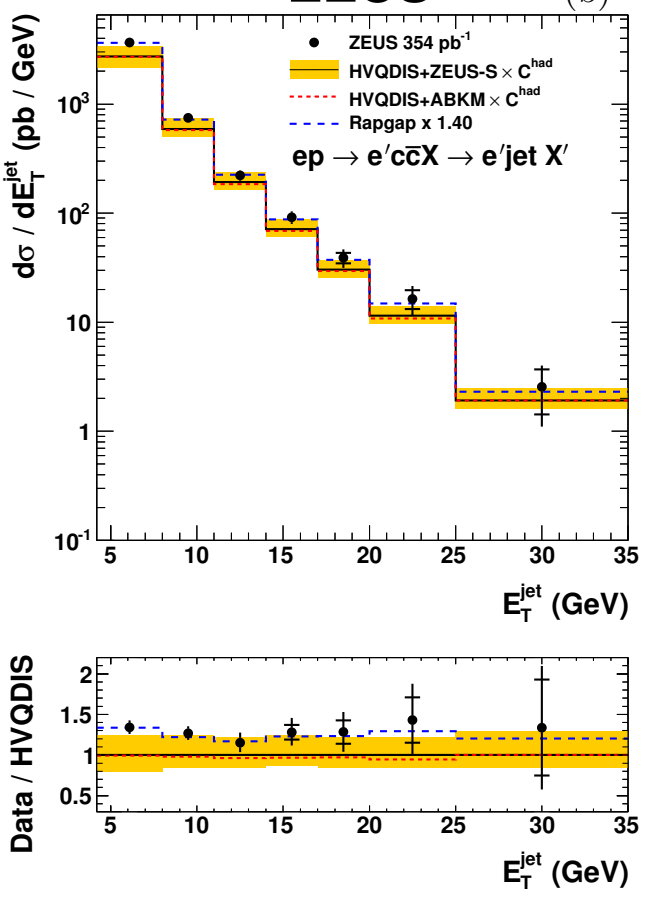

Figure 5. Differential cross sections for inclusive jet production in (a) beauty events and (b) charm events as a function of $E_{T}^{\text {jet }}$. The cross sections are given for $5<Q^{2}<1000 \mathrm{GeV}^{2}, 0.02<y<0.7$, $E_{T}^{\text {jet }}>5(4.2) \mathrm{GeV}$ and $-1.6<\eta^{\text {jet }}<2.2$. The data are shown as points. The inner error bars are the statistical uncertainties, while the outer error bars show the statistical and systematic uncertainties (not including the error on the integrated luminosity) added in quadrature. The solid line shows the HVQDIS prediction with the ZEUS-S PDF, corrected for hadronisation effects, with the uncertainties indicated by the band; the dotted line shows the same prediction using the ABKM PDF; the dashed line shows the prediction from RAPGAP scaled to match the measured integrated cross sections. 


\section{ZEUS}
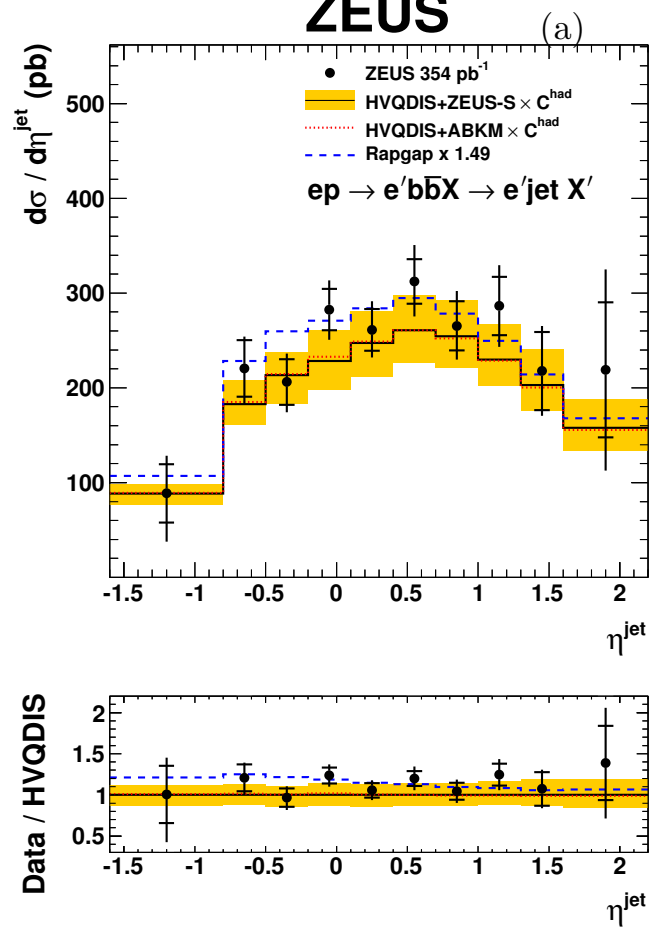

ZEUS
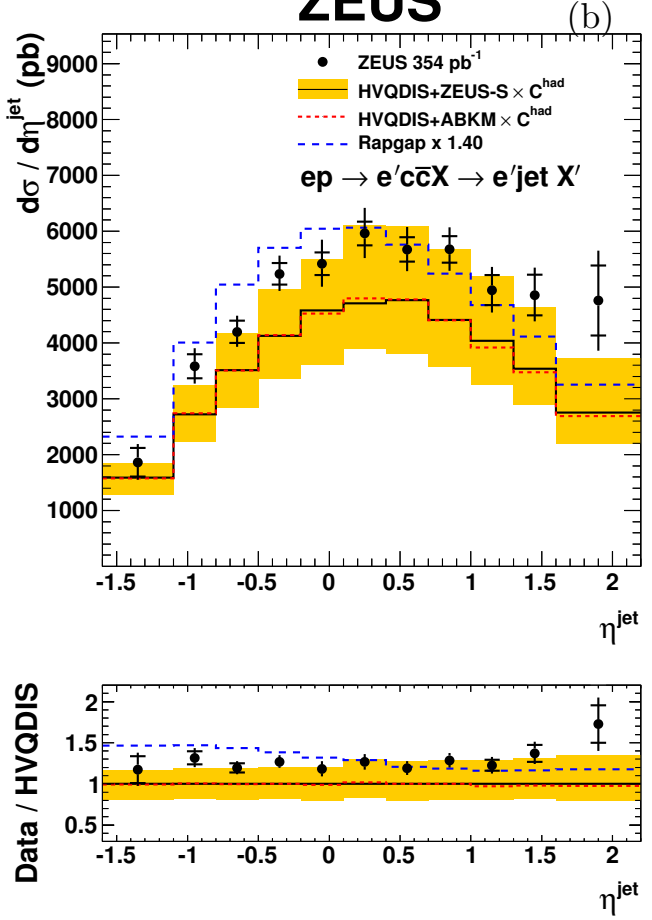

Figure 6. Differential cross sections for inclusive jet production in (a) beauty events and (b) charm events as a function of $\eta^{\text {jet }}$. For more details, see the caption of figure 5 .
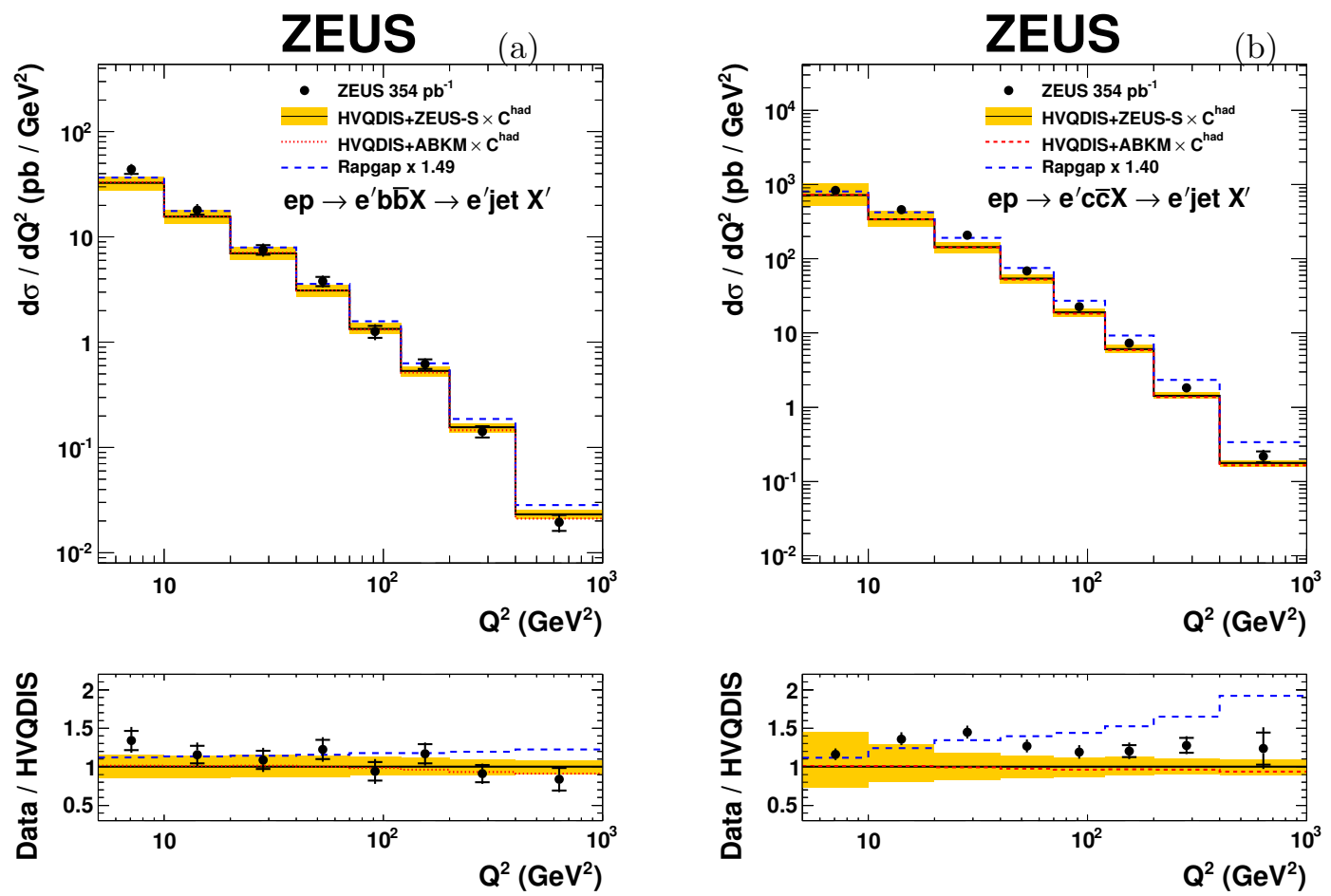

Figure 7. Differential cross sections for inclusive jet production in (a) beauty events and (b) charm events as a function of $Q^{2}$. For more details, see the caption of figure 5 . 

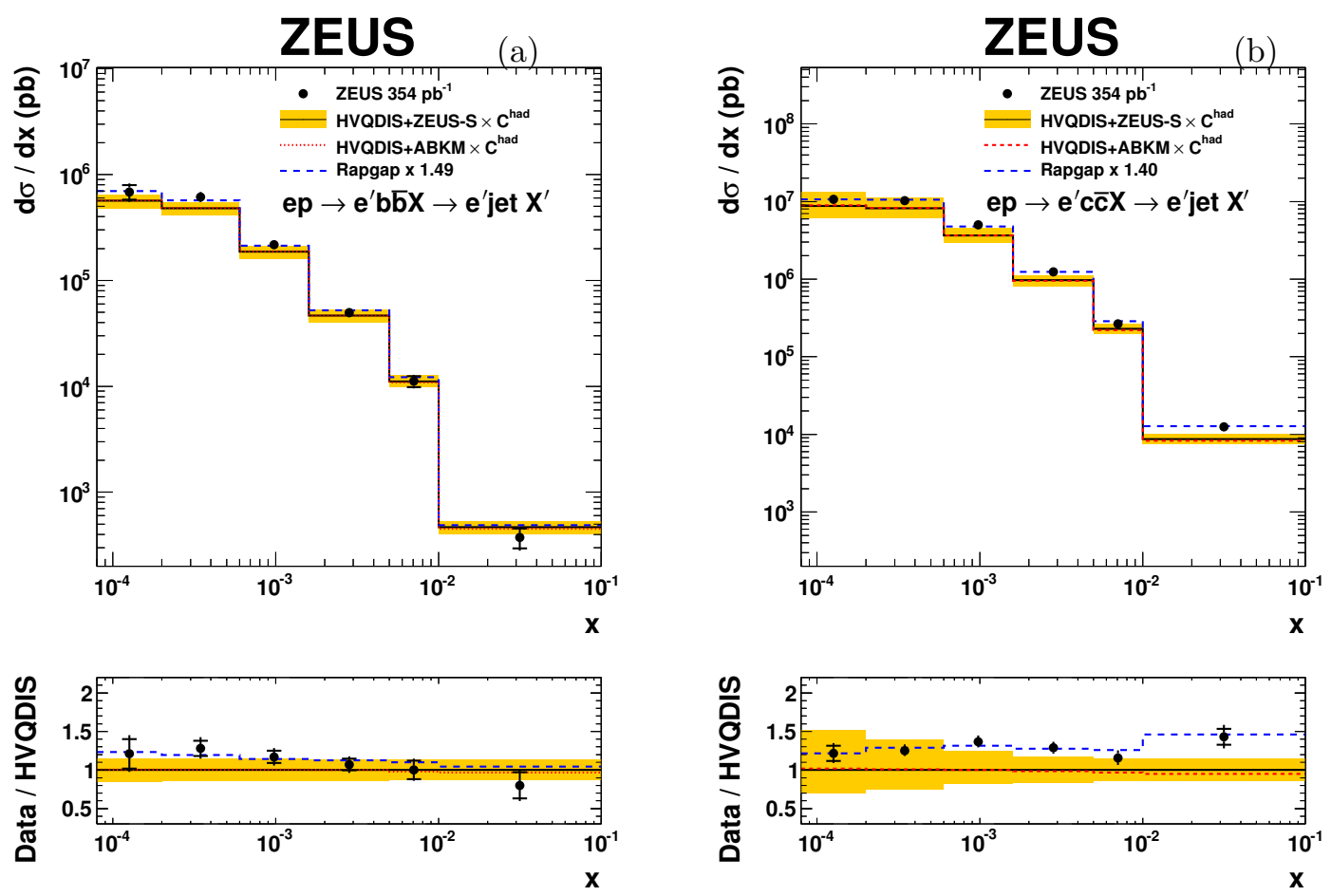

Figure 8. Differential cross sections for inclusive jet production in (a) beauty events and (b) charm events as a function of $x$. For more details, see the caption of figure 5 . 


\section{ZEUS}

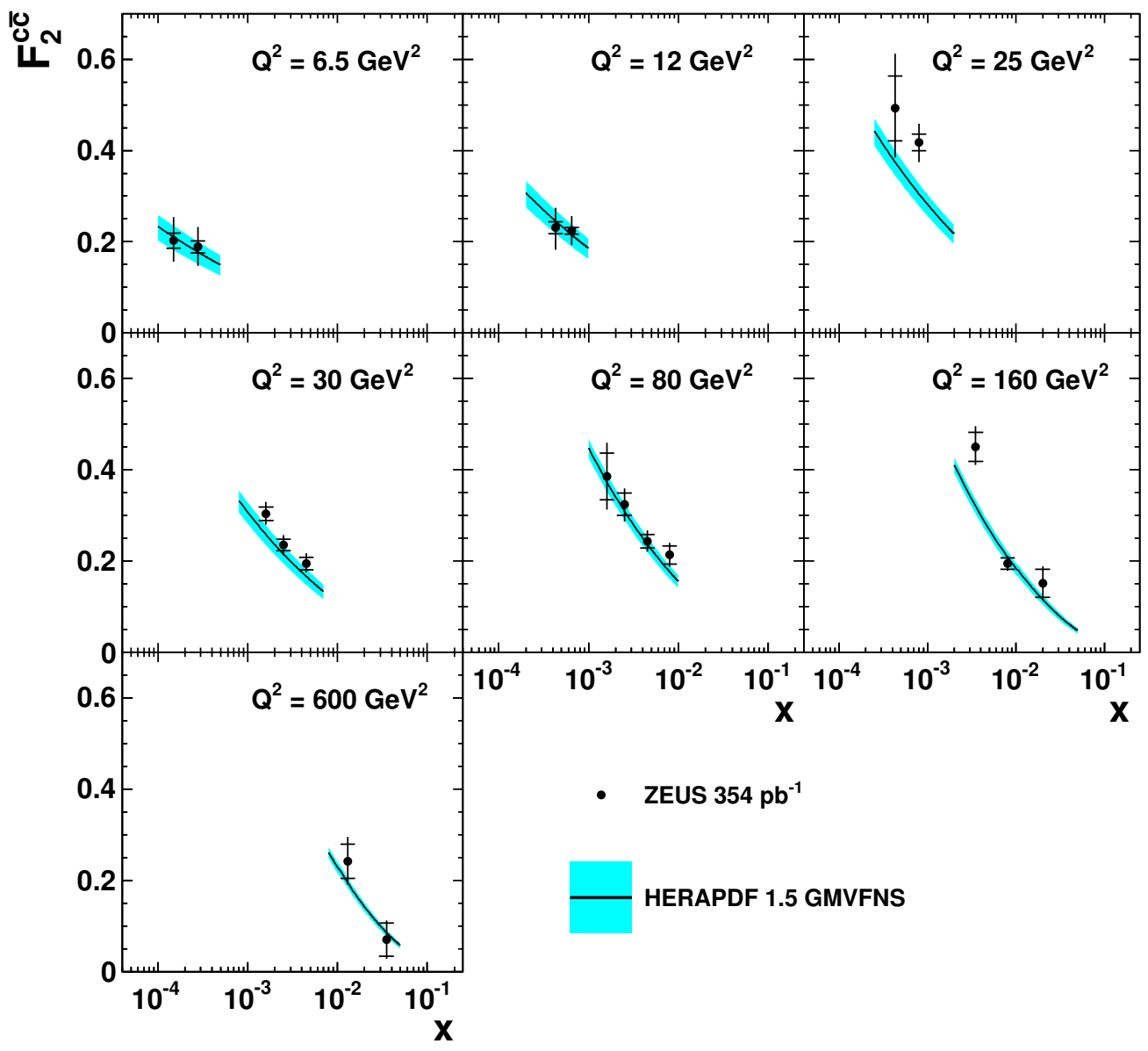

Figure 9. The structure function $F_{2}^{c \bar{c}}$ (filled symbols) as a function of $x$ for seven different values of $Q^{2}$. The inner error bars are the statistical uncertainty while the outer error bars represent the statistical, systematic (not including the error on the integrated luminosity) and extrapolation uncertainties added in quadrature. Also shown are the NLO QCD HERAPDF 1.5 predictions based on the general-mass variable-flavour-number scheme (solid line and shaded area for the uncertainties). 


\section{ZEUS}

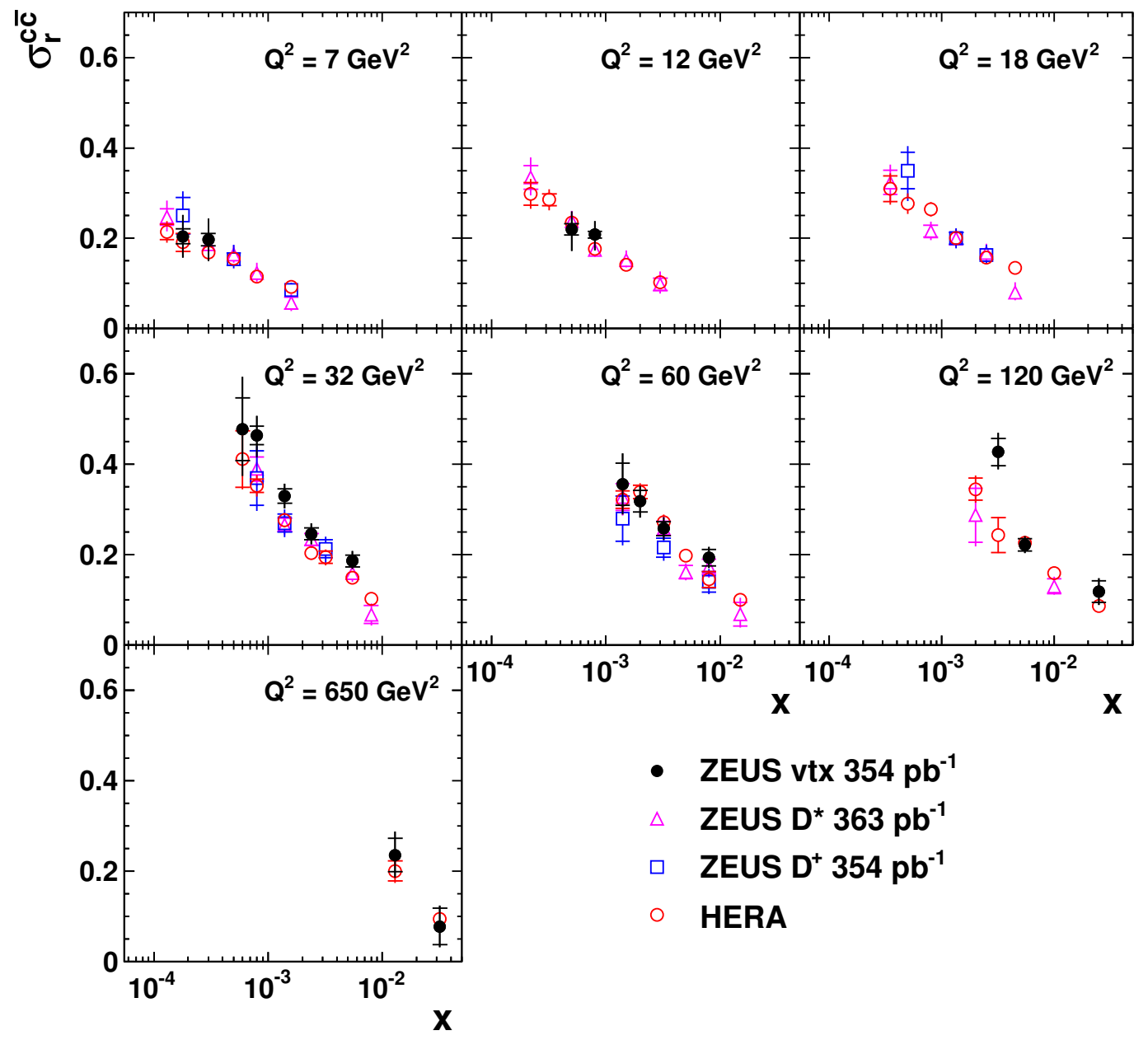

Figure 10. Reduced charm cross section, $\sigma_{\mathrm{r}}^{c \bar{c}}$, as a function of $x$ for fixed values of $Q^{2}$. Results from the current analysis (filled circles) are compared to the ZEUS $D^{* \pm}$ data [31] (empty triangles), the ZEUS $D^{+}$measurement [30] (empty squares) and the combination of previous HERA results [32] (empty circles). The inner error bars in the ZEUS measurements show the statistical uncertainties. The inner error bars of the combined HERA data represent the uncorrelated part of the uncertainty. The outer error bars include statistical, systematic (not including the error on the integrated luminosity) and theoretical uncertainties added in quadrature. 


\section{ZEUS}

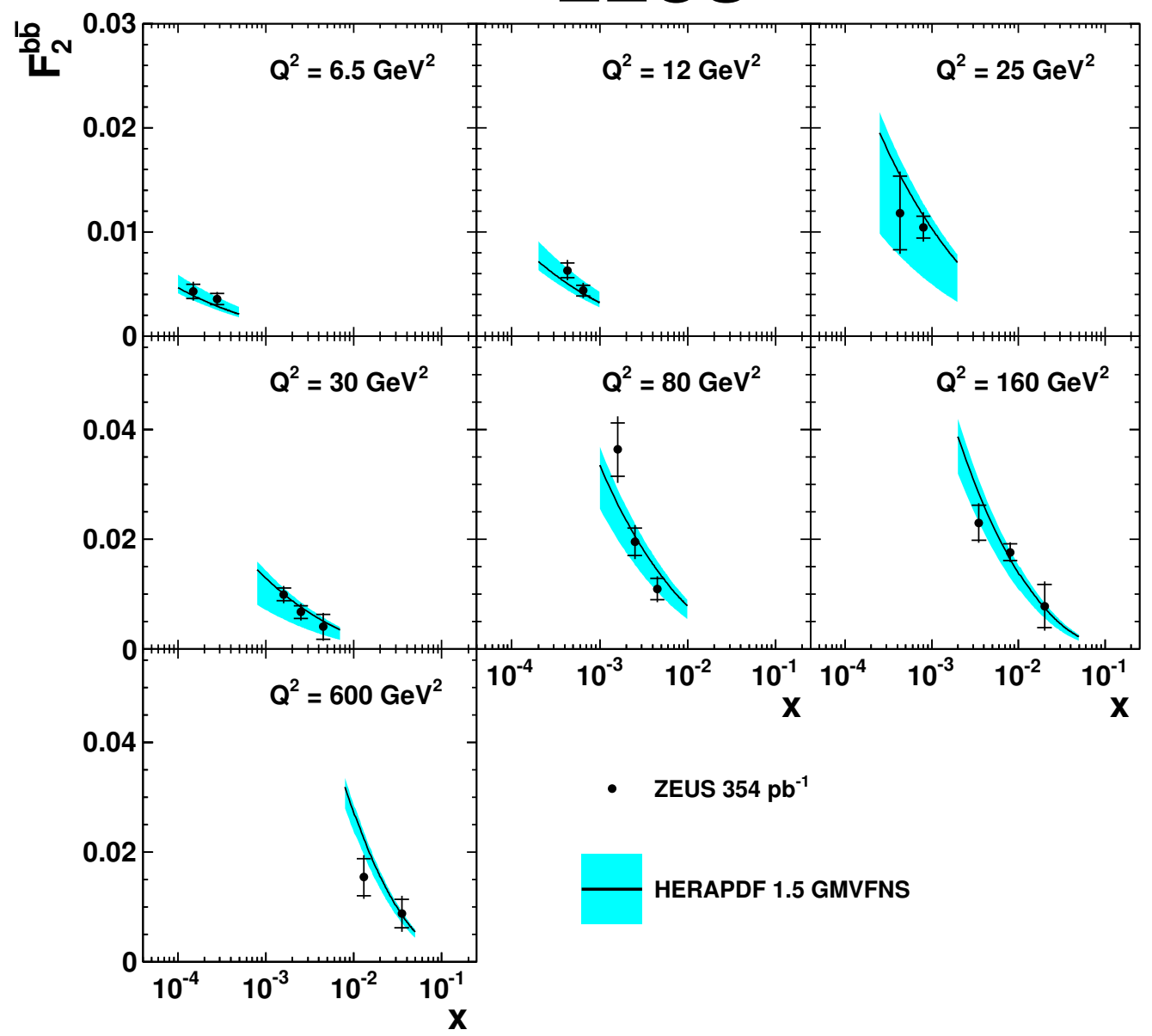

Figure 11. The structure function $F_{2}^{b \bar{b}}$ (filled symbols) as a function of $x$ for seven different values of $Q^{2}$. The inner error bars are the statistical uncertainty while the outer error bars represent the statistical, systematic (not including the error on the integrated luminosity) and extrapolation uncertainties added in quadrature. Also shown are the NLO QCD HERAPDF 1.5 predictions based on the general-mass variable-flavour-number scheme (solid line and shaded area for the uncertainties). 


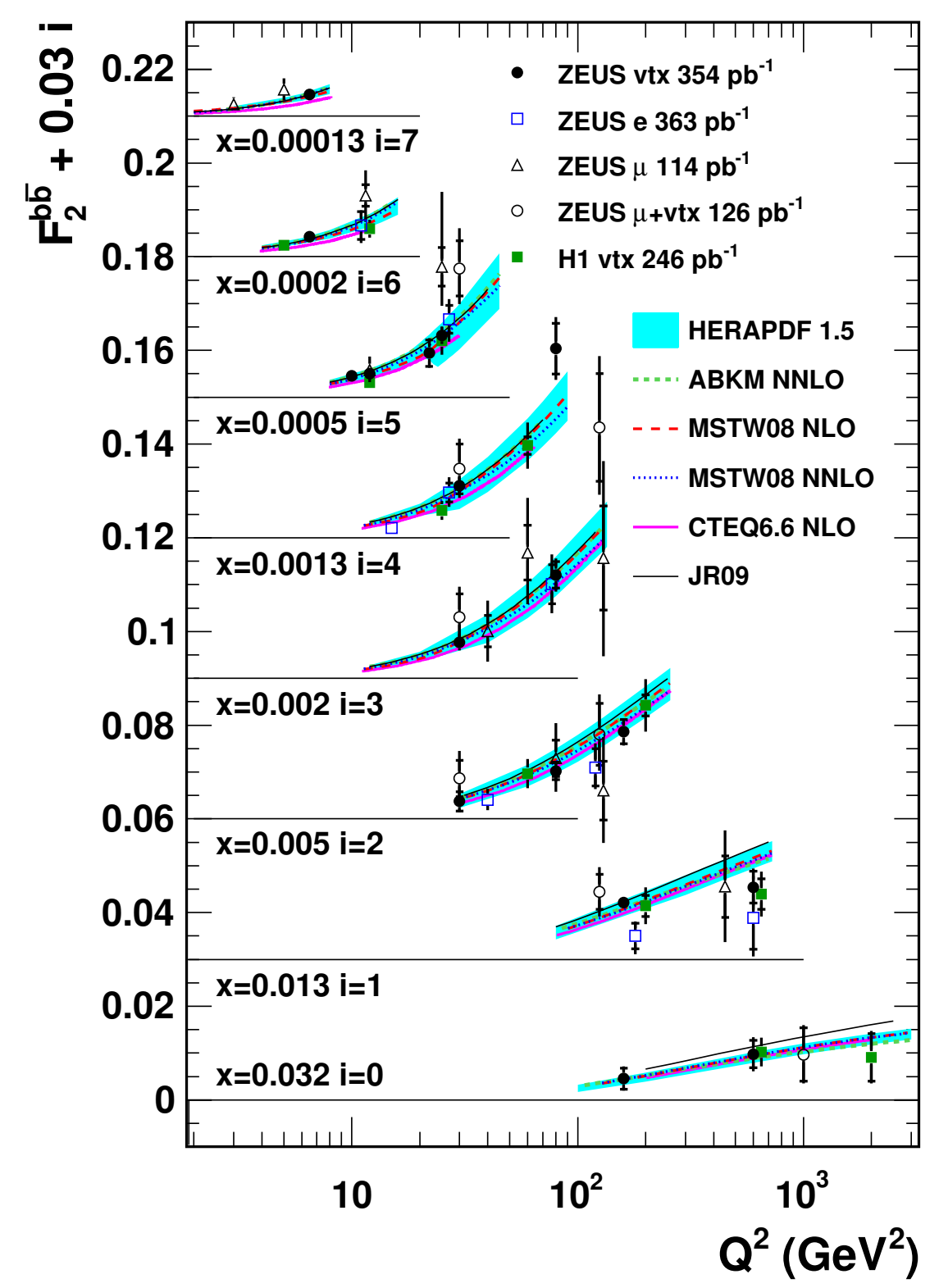

Figure 12. The structure function $F_{2}^{b \bar{b}}$ (filled circles) as a function of $Q^{2}$ for fixed values of $x$ compared to previous results (open squares [29], open triangles [27], open circles [26] and filled squares $[9,11,14])$. The inner error bars are the statistical uncertainty while the outer error bars represent the statistical, systematic (not including the error on the integrated luminosity) and extrapolation uncertainties added in quadrature. The data have been corrected to the same reference $x$ as the previous analysis [27]. The measurements are compared to several NLO and NNLO QCD predictions [62, 84-90]. 


\section{ZEUS}

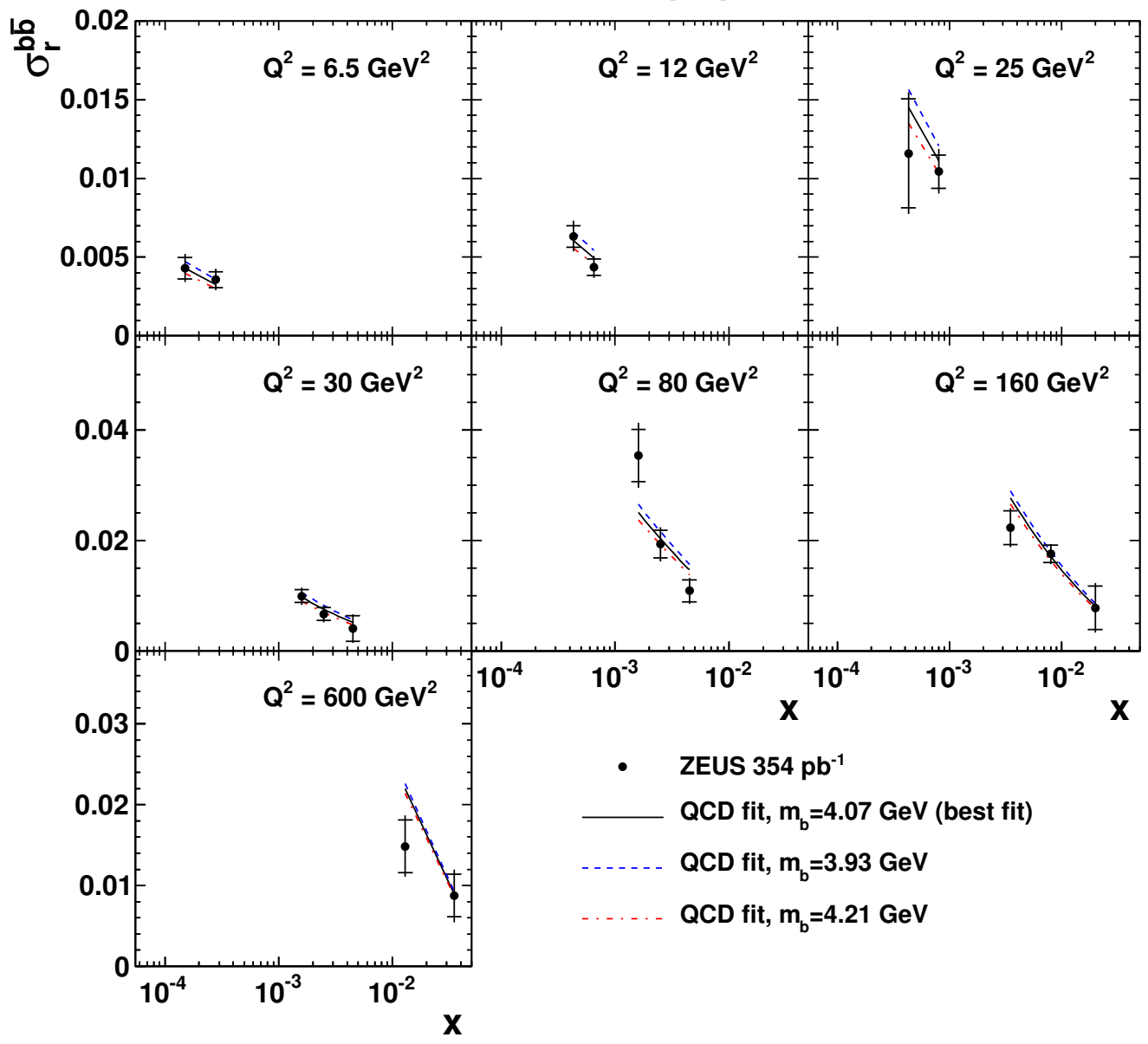

Figure 13. Reduced beauty cross section, $\sigma_{\mathrm{r}}^{b \bar{b}}$, (filled symbols) as a function of $x$ for seven different values of $Q^{2}$. The inner error bars are the statistical uncertainty while the outer error bars represent the statistical, systematic (not including the error on the integrated luminosity) and extrapolation uncertainties added in quadrature. Also shown are the results of the QCD fit described in section 10. The central line indicates the best fit, the lower and upper line give the fit for a higher and lower beauty mass, respectively. 


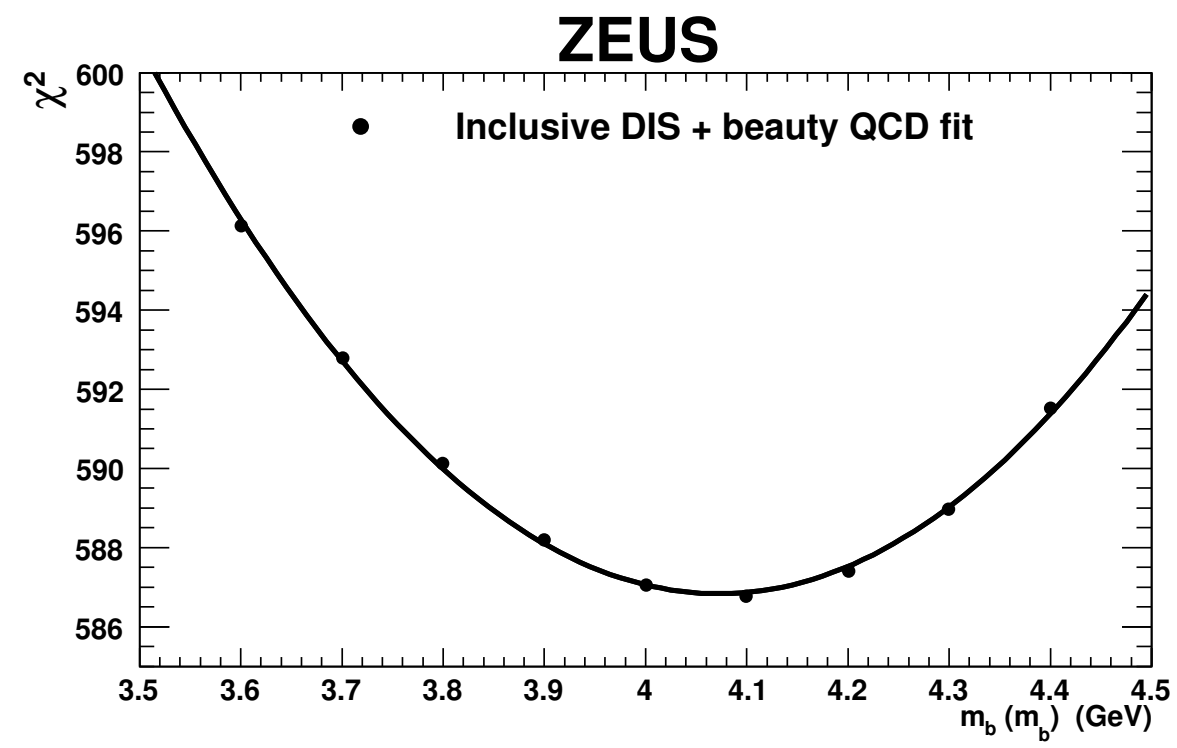

Figure 14. The values of $\chi^{2}$ for the PDF fit to the combined HERA DIS data including the beauty measurements, as a function of the running beauty quark mass $m_{b}\left(m_{b}\right)$. The FFNS ABM scheme is used, where the beauty quark mass is defined in the $\overline{\mathrm{MS}}$ scheme. The solid line is a second order polynomial parameterisation of the points.

\begin{tabular}{|ll|c|c|}
\hline Source & $\begin{array}{c}\text { Beauty } \\
(\%)\end{array}$ & $\begin{array}{c}\text { Charm } \\
(\%)\end{array}$ \\
\hline$\delta_{1}$ & Event and DIS selection & \pm 1.4 & \pm 0.8 \\
\hline$\delta_{2}$ & Trigger efficiency & +2.0 & +1.0 \\
\hline$\delta_{3}$ & Tracking efficiency & \pm 2.0 & \pm 0.5 \\
\hline$\delta_{4}$ & Decay-length smearing & \pm 1.3 & \pm 1.2 \\
\hline$\delta_{5}$ & Signal extraction procedure & \pm 0.8 & \pm 0.8 \\
\hline$\delta_{6}$ & Jet energy scale & \pm 0.7 & \pm 0.9 \\
\hline$\delta_{7}$ & EM energy scale & \pm 0.3 & \pm 0.1 \\
\hline$\delta_{8}$ & Charm $Q^{2}$ reweighting $\left(\delta_{8}^{Q^{2}, c}\right)$ & \pm 1.7 & \pm 1.8 \\
& Beauty $Q^{2}$ reweighting $\left(\delta_{8}^{Q^{2}, b}\right)$ & \pm 2.9 & \pm 0.4 \\
& Charm $\eta^{\text {jet }}$ reweighting $\left(\delta_{8}^{\text {jet }}, c\right.$ \\
& Beauty $\eta^{\text {jet }}$ reweighting $\left(\delta_{8}^{\text {jet }, b}\right)$ & ${ }_{-0.4}^{+0.3}$ & ${ }_{-1.0}^{+1.5}$ \\
& ${ }_{-0.4}^{+0.7}$ & ${ }_{-0.1}^{+0.0}$ \\
& Charm $E_{T}^{\text {jet }}$ reweighting $\left(\delta_{8}^{E_{T}^{\text {jet }}, c}\right)$ & ${ }_{-1.3}^{+1.7}$ & ${ }_{-1.7}^{+2.2}$ \\
& Beauty $E_{T}^{\text {jet }}$ reweighting $\left(\delta_{8}^{E_{T}^{\text {jet }}, b}\right)$ & ${ }_{-4.2}^{+5.4}$ & ${ }_{-0.6}^{+0.5}$ \\
\hline$\delta_{9}$ & Light-flavour asymmetry & \pm 0.4 & \pm 2.0 \\
\hline$\delta_{10}$ & Charm fragmentation function & -0.9 & +1.0 \\
\hline$\delta_{11}$ & Beauty fragmentation function & -3.1 & +0.0 \\
\hline$\delta_{12}$ & BR and fragmentation fractions & ${ }_{-2.1}^{+1.8}$ & ${ }_{-2.6}^{+3.5}$ \\
\hline$\delta_{13}$ & Luminosity measurement & \pm 1.9 & \pm 1.9 \\
\hline & Total & ${ }_{-7.6}^{+8.0}$ & ${ }_{-5.1}^{+6.0}$ \\
\hline
\end{tabular}

Table 1. Effects of the systematic uncertainties on the integrated beauty- and charm-jet cross sections. 


\begin{tabular}{|c|c|c|c|c|}
\hline $\begin{array}{c}E_{T}^{\mathrm{jet}} \\
(\mathrm{GeV})\end{array}$ & $\begin{array}{r}\mathrm{d} \sigma_{b}^{\mathrm{jet}} / \mathrm{d} E_{T}^{\mathrm{jet}}(\mathrm{pb} / \mathrm{C} \\
\text { stat. }\end{array}$ & $\begin{array}{l}\mathrm{GeV}) \\
\text { syst. }\end{array}$ & $C^{\text {had }}$ & $C^{\mathrm{rad}}$ \\
\hline $5: 8$ & \pm 26 & $\begin{array}{l}+24 \\
-25\end{array}$ & 0.95 & 1.01 \\
\hline $8: 11$ & $66.1 \pm 5.8$ & $\begin{array}{l}+5.1 \\
-6.8\end{array}$ & 1.08 & 0.98 \\
\hline $11: 14$ & $30.1 \pm 1.9$ & $\begin{array}{l}+1.4 \\
-2.0\end{array}$ & 1.05 & 0.96 \\
\hline $14: 17$ & $11.27 \pm 0.90$ & ${ }_{-0.60}^{+0.52}$ & 1.04 & 0.95 \\
\hline $17: 20$ & $4.71 \pm$ & $\begin{array}{l}+0.34 \\
-0.32\end{array}$ & 0.99 & 0.93 \\
\hline $20: 25$ & $2.04 \pm$ & $\begin{array}{l}+0.28 \\
-0.28\end{array}$ & 0.93 & 0.89 \\
\hline $25 \quad: 35$ & $0.380 \pm \quad 0.094$ & $1_{-0.076}^{+0.078}$ & 0.80 & 0.89 \\
\hline
\end{tabular}

\begin{tabular}{|c|c|c|c|c|c|}
\hline $\begin{array}{c}E_{T}^{\mathrm{jet}} \\
(\mathrm{GeV})\end{array}$ & \multicolumn{3}{|c|}{$\mathrm{d} \sigma_{c}^{\mathrm{jet}} / \mathrm{d} E_{T}^{\mathrm{jet}}(\mathrm{pb} / \mathrm{GeV})$} & $C^{\text {had }}$ & $C^{\mathrm{rad}}$ \\
\hline $4.2: 8$ & 3660 & \pm 120 & ${ }_{-180}^{+200}$ & 1.06 & 0.98 \\
\hline $8: 11$ & 748 & \pm 22 & $\begin{array}{l}+45 \\
-41\end{array}$ & 1.05 & 0.97 \\
\hline $11: 14$ & 222 & \pm 10 & ${ }_{-20}^{+21}$ & 1.03 & 0.96 \\
\hline $14: 17$ & 91.4 & $\pm \quad 6.5$ & $\begin{array}{l}+11 \\
-9.7\end{array}$ & 0.99 & 0.93 \\
\hline $17: 20$ & 38.9 & $\pm \quad 4.4$ & $\begin{array}{l}+6.1 \\
-6.0\end{array}$ & 0.96 & 0.93 \\
\hline $20: 25$ & 16.4 & $\pm \quad 3.2$ & $\begin{array}{l}+4.0 \\
-3.7\end{array}$ & 0.95 & 0.85 \\
\hline $25 \quad: 35$ & 2.6 & $\pm \quad 1.1$ & $\begin{array}{l}+0.9 \\
-0.9\end{array}$ & 0.86 & 0.88 \\
\hline
\end{tabular}

Table 2. Differential cross sections for inclusive jet production in beauty events (top) and charm events (bottom) as a function of $E_{T}^{\text {jet }}$. The beauty (charm) cross sections are given for $5<Q^{2}<$ $1000 \mathrm{GeV}^{2}, 0.02<y<0.7, E_{T}^{\text {jet }}>5(4.2) \mathrm{GeV}$ and $-1.6<\eta^{\text {jet }}<2.2$. The measurements are given together with their statistical and systematic uncertainties (not including the error on the integrated luminosity). Hadronisation and QED radiative corrections, $C^{\text {had }}$ and $C^{\text {rad }}$, respectively, are also shown. 


\begin{tabular}{|c|c|c|c|c|c|}
\hline$\eta^{\mathrm{jet}}$ & \multicolumn{3}{|c|}{$\begin{array}{r}\mathrm{d} \sigma_{b}^{\text {jet }} / \mathrm{d} \eta^{\text {jet }}(\mathrm{pb}) \\
\text { stat. } \quad \text { syst. }\end{array}$} & $C^{\text {had }}$ & $C^{\mathrm{rad}}$ \\
\hline$-1.6:-0.8$ & $89 \pm$ & 31 & $\begin{array}{l}+25 \\
-41\end{array}$ & 0.96 & 0.98 \\
\hline$-0.8:-0.5$ & $220 \pm$ & 30 & $\begin{array}{l}+15 \\
-23\end{array}$ & 0.98 & 0.98 \\
\hline$-0.5:-0.2$ & $210 \pm$ & 24 & $\begin{array}{l}+18 \\
-21\end{array}$ & 0.93 & 0.99 \\
\hline$-0.2: \quad 0.1$ & $280 \pm$ & 22 & $\begin{array}{l}+21 \\
-23\end{array}$ & 0.91 & 0.99 \\
\hline $0.1: \quad 0.4$ & $260 \pm$ & 22 & $\begin{array}{l}+21 \\
{ }_{-19}\end{array}$ & 0.94 & 0.99 \\
\hline $0.4: \quad 0.7$ & $310 \pm$ & 23 & $\begin{array}{l}+30 \\
-29\end{array}$ & 1.01 & 0.99 \\
\hline $0.7: 1.0$ & 270 & 26 & $\begin{array}{l}+26 \\
-24\end{array}$ & 1.06 & 0.99 \\
\hline $1.0: 1.3$ & $290 \pm$ & 31 & $\begin{array}{l}+30 \\
-30\end{array}$ & 1.07 & 0.99 \\
\hline $1.3: 1.6$ & $220 \pm$ & 41 & $\begin{array}{l}+24 \\
-23\end{array}$ & 1.07 & 0.99 \\
\hline $1.6: \quad 2.2$ & $220 \pm$ & 71 & $\begin{array}{l}+78 \\
{ }_{-79}\end{array}$ & 1.07 & 0.98 \\
\hline
\end{tabular}

\begin{tabular}{|c|c|c|c|c|c|}
\hline$\eta^{\text {jet }}$ & \multicolumn{3}{|c|}{$\begin{array}{r}\mathrm{d} \sigma_{c}^{\text {jet }} / \mathrm{d} \eta^{\text {jet }}(\mathrm{pb}) \\
\text { stat. } \quad \text { syst. }\end{array}$} & $C^{\text {had }}$ & $C^{\mathrm{rad}}$ \\
\hline$-1.6:-1.1$ & $1900 \pm$ & 260 & $\begin{array}{l}+200 \\
{ }_{-180}\end{array}$ & 0.89 & 0.99 \\
\hline$-1.1:-0.8$ & $3600 \pm$ & 220 & $\begin{array}{l}+240 \\
-220\end{array}$ & 0.97 & 0.98 \\
\hline$-0.8:-0.5$ & $4200 \pm$ & 200 & $\begin{array}{l}+210 \\
-180\end{array}$ & 1.02 & 0.98 \\
\hline$-0.5:-0.2$ & $5200 \pm$ & 190 & $\begin{array}{l}+260 \\
-240\end{array}$ & 1.05 & 0.98 \\
\hline$-0.2: \quad 0.1$ & $5400 \pm$ & 200 & $\begin{array}{l}+380 \\
{ }_{-360}\end{array}$ & 1.07 & 0.98 \\
\hline $0.1: \quad 0.4$ & $6000 \pm$ & 210 & $\begin{array}{l}+410 \\
-380\end{array}$ & 1.10 & 0.98 \\
\hline $0.4: \quad 0.7$ & $5700 \pm$ & 220 & $\begin{array}{l}+340 \\
{ }_{-320}\end{array}$ & 1.11 & 0.98 \\
\hline $0.7: 1.0$ & $5700 \pm$ & 240 & $\begin{array}{l}+320 \\
-300\end{array}$ & 1.10 & 0.98 \\
\hline $1.0: \quad 1.3$ & $4900 \pm$ & 270 & $\begin{array}{l}+320 \\
{ }_{-300}\end{array}$ & 1.09 & 0.98 \\
\hline $1.3: 1.6$ & $4900 \pm$ & 360 & $\begin{array}{l}+330 \\
-300\end{array}$ & 1.07 & 0.97 \\
\hline $1.6: \quad 2.2$ & $4800 \pm$ & 630 & $\begin{array}{l}+640 \\
-640 \\
\end{array}$ & 1.13 & 0.97 \\
\hline
\end{tabular}

Table 3. Differential cross sections for inclusive jet production in beauty events (top) and charm events (bottom) as a function of $\eta^{\text {jet }}$. For details, see the caption of table 2. 


\begin{tabular}{|c|c|c|c|c|c|}
\hline $\begin{array}{c}Q^{2} \\
\left(\mathrm{GeV}^{2}\right)\end{array}$ & $\mathrm{d} \sigma_{b}^{\mathrm{jet}} / \mathrm{d} Q^{2}$ & $\begin{array}{l}\text { (pb/Ge } \\
\text { stat. }\end{array}$ & $\begin{array}{l}\left.\mathrm{eV}^{2}\right) \\
\text { syst. }\end{array}$ & $C^{\text {had }}$ & $C^{\mathrm{rad}}$ \\
\hline 5: 10 & 43.8 & 4.1 & $\begin{array}{l}+4.0 \\
{ }_{-3.2}\end{array}$ & 1.01 & 0.99 \\
\hline 10: 20 & 18.0 & 1.8 & ${ }_{-1.5}^{+1.7}$ & 1.01 & 0.99 \\
\hline $20: \quad 40$ & $7.58 \pm$ & 0.82 & ${ }_{-0.72}^{+0.74}$ & 0.99 & 0.99 \\
\hline $40: \quad 70$ & $3.80 \pm$ & 0.39 & $\begin{array}{l}+0.30 \\
{ }_{-0.31}\end{array}$ & 0.98 & 0.99 \\
\hline $70: 120$ & $1.26 \pm$ & 0.16 & $\begin{array}{l}+0.14 \\
{ }_{-0.15}\end{array}$ & 0.98 & 0.98 \\
\hline $120: 200$ & $0.623 \pm$ & 0.066 & $\begin{array}{l}+0.042 \\
+0.047\end{array}$ & 0.99 & 0.99 \\
\hline $200: 400$ & $0.142 \pm$ & 0.018 & $\begin{array}{l}+0.010 \\
{ }_{-0.010}\end{array}$ & 0.99 & 0.99 \\
\hline $400: 1000$ & $0.0194 \pm$ & 0.0034 & $4_{-0.0019}^{+0.0020}$ & 1.01 & 0.95 \\
\hline
\end{tabular}

\begin{tabular}{|c|c|c|c|c|c|c|}
\hline $\begin{array}{c}Q^{2} \\
\left(\mathrm{GeV}^{2}\right)\end{array}$ & \multicolumn{4}{|c|}{$\mathrm{d} \sigma_{c}^{\mathrm{jet}} / \mathrm{d} Q^{2}\left(\mathrm{pb} / \mathrm{GeV}^{2}\right)$} & $C^{\text {had }}$ & $C^{\mathrm{rad}}$ \\
\hline 5: 10 & 835 & \pm & 34 & $\begin{array}{l}+46 \\
-39\end{array}$ & 1.15 & 0.98 \\
\hline 10: 20 & 460 & \pm & 15 & $\begin{array}{l}+26 \\
-22\end{array}$ & 1.08 & 0.99 \\
\hline $20: \quad 40$ & 207 & \pm & 6.4 & $\begin{array}{l}+10 \\
-9.5\end{array}$ & 1.01 & 0.98 \\
\hline 40: 70 & 68.5 & \pm & 2.7 & $\begin{array}{l}+3.8 \\
-3.5\end{array}$ & 1.00 & 0.97 \\
\hline $70: 120$ & 22.5 & \pm & 1.0 & $\begin{array}{l}+1.4 \\
-1.2\end{array}$ & 1.00 & 0.97 \\
\hline $120: 200$ & 7.28 & \pm & 0.46 & ${ }_{-0.41}^{+0.48}$ & 1.01 & 0.96 \\
\hline $200: 400$ & 1.82 & \pm & 0.14 & $\begin{array}{l}+0.10 \\
-0.08\end{array}$ & 1.01 & 0.95 \\
\hline $400: 1000$ & 0.219 & \pm & 0.037 & $\begin{array}{l}+0.032 \\
{ }_{-0.029}\end{array}$ & 1.02 & 0.87 \\
\hline
\end{tabular}

Table 4. Differential cross sections for inclusive jet production in beauty events (top) and charm events (bottom) as a function of $Q^{2}$. For details, see the caption of table 2. 


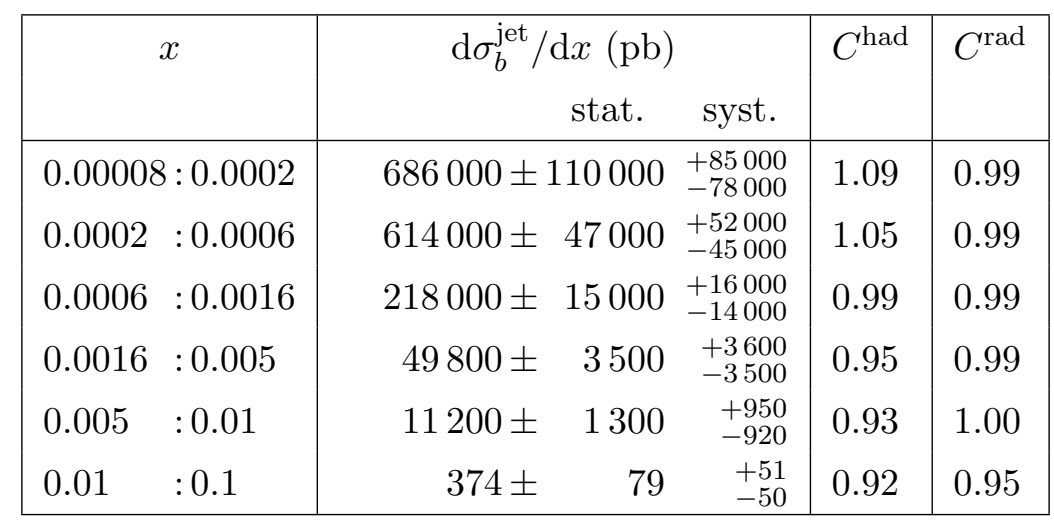

\begin{tabular}{|c|c|c|c|c|c|}
\hline \multicolumn{2}{|r|}{$x$} & \multicolumn{2}{|l|}{$\mathrm{d} \sigma_{c}^{\mathrm{jet}} / \mathrm{d} x(\mathrm{pb})$} & $C^{\text {had }}$ & $C^{\mathrm{rad}}$ \\
\hline 0.00008 & $3: 0.0$ & $10700000 \pm 870000$ & $\begin{array}{l}+760000 \\
-650000\end{array}$ & 1.19 & 0.96 \\
\hline 0.0002 & : 0.0006 & $10300000 \pm 390000$ & & 1.20 & 0.98 \\
\hline 0.0006 & $: 0.0016$ & $4990000 \pm 140000$ & & 1.09 & 0.99 \\
\hline 0.0016 & $: 0.005$ & $1250000 \pm 32000$ & & 0.97 & 0.99 \\
\hline 0.005 & $: 0.01$ & $264000 \pm 12000$ & ${ }_{-17}^{+19}$ & 0.91 & 1.00 \\
\hline 0.01 & $: 0.1$ & $12500 \pm$ & ${ }_{-970}^{+1000}$ & 0.88 & 0.88 \\
\hline
\end{tabular}

Table 5. Differential cross sections for inclusive jet production in beauty events (top) and charm events (bottom) as a function of $x$. For details, see the caption of table 2. 


\begin{tabular}{|c|c|c|c|c|c|c|c|}
\hline $\begin{array}{c}Q^{2} \\
\left(\mathrm{GeV}^{2}\right)\end{array}$ & & $x$ & $\mathrm{~d}^{2} \sigma_{b}^{\text {jet }} / \mathrm{d} x$ & $\begin{array}{l}\mathrm{d} Q^{2}(\mathrm{pb} \\
\text { stat. }\end{array}$ & $\begin{array}{l}\left(\mathrm{GeV}^{2}\right) \\
\text { syst. }\end{array}$ & $C^{\text {had }}$ & $C^{\mathrm{rad}}$ \\
\hline $5: \quad 20$ & 0.00008 & $8: 0.0002$ & $690000 \pm$ & 110000 & $\begin{array}{l}+90000 \\
-80000\end{array}$ & 1.09 & 0.99 \\
\hline $5: \quad 20$ & 0.0002 & $: 0.0003$ & $830000 \pm$ & 120000 & $\begin{array}{l}+80000 \\
{ }_{-80000}\end{array}$ & 1.07 & 0.98 \\
\hline $5: \quad 20$ & 0.0003 & $: 0.0005$ & $501000 \pm$ & 55000 & $\begin{array}{l}+49000 \\
-41000\end{array}$ & 1.04 & 0.99 \\
\hline $5: \quad 20$ & 0.0005 & $: 0.003$ & $48200 \pm$ & 5800 & $\begin{array}{l}+5100 \\
-4700\end{array}$ & 0.91 & 0.99 \\
\hline $20: \quad 60$ & 0.0003 & $: 0.0005$ & $82000 \pm$ & 24000 & $\begin{array}{l}+11000 \\
-11000\end{array}$ & 1.07 & 0.98 \\
\hline $20: \quad 60$ & 0.0005 & $: 0.0012$ & $134000 \pm$ & 14000 & $\begin{array}{l}+10000 \\
-10000\end{array}$ & 1.05 & 0.99 \\
\hline $20: \quad 60$ & 0.0012 & $: 0.002$ & $73400 \pm$ & 8500 & $\begin{array}{l}+6900 \\
-6900\end{array}$ & 1.00 & 1.00 \\
\hline $20: \quad 60$ & 0.002 & : 0.0035 & $25800 \pm$ & 4600 & $\begin{array}{l}+3500 \\
-3400\end{array}$ & 0.94 & 1.01 \\
\hline $20: \quad 60$ & 0.0035 & $: 0.01$ & $3600 \pm$ & 2000 & $\begin{array}{l}+1000 \\
-1000 \\
\end{array}$ & 0.81 & 0.99 \\
\hline $60: 120$ & 0.0008 & $: 0.0018$ & $33400 \pm$ & 4500 & $\begin{array}{l}+3200 \\
-3100\end{array}$ & 1.03 & 0.98 \\
\hline $60: 120$ & 0.0018 & $: 0.003$ & $22500 \pm$ & 2900 & $\begin{array}{l}+2000 \\
-2100\end{array}$ & 1.02 & 0.99 \\
\hline $60: 120$ & 0.003 & $: 0.006$ & $7400 \pm$ & 1300 & $\begin{array}{l}+800 \\
-900\end{array}$ & 0.98 & 0.98 \\
\hline $120: 400$ & 0.0016 & $: 0.005$ & $6700 \pm$ & 930 & $\begin{array}{l}+450 \\
-500\end{array}$ & 1.01 & 0.99 \\
\hline $120: 400$ & 0.005 & $: 0.016$ & $3820 \pm$ & 340 & $\begin{array}{l}+170 \\
{ }_{-200}\end{array}$ & 0.99 & 1.02 \\
\hline $120: 400$ & 0.016 & $: 0.06$ & $269 \pm$ & 130 & $\begin{array}{l}+70 \\
-80 \\
\end{array}$ & 0.92 & 0.87 \\
\hline $400: 1000$ & 0.005 & $: 0.02$ & $401 \pm$ & 88 & $\begin{array}{l}+56 \\
-53\end{array}$ & 1.01 & 0.95 \\
\hline $400: 1000$ & 0.02 & $: 0.1$ & $70 \pm$ & 21 & $\begin{array}{l}+15 \\
-16 \\
\end{array}$ & 1.00 & 0.95 \\
\hline
\end{tabular}

Table 6. Double-differential cross sections for inclusive jet production in beauty events as a function of $x$ for different ranges of $Q^{2}$. The cross sections are given for $5<Q^{2}<1000 \mathrm{GeV}^{2}, 0.02<y<0.7$, $E_{T}^{\text {jet }}>5 \mathrm{GeV}$ and $-1.6<\eta^{\text {jet }}<2.2$. The measurements are given together with their statistical and systematic uncertainties (not including the error on the integrated luminosity). Hadronisation and QED radiative corrections, $C^{\text {had }}$ and $C^{\text {rad }}$, respectively, are also shown. 


\begin{tabular}{|c|c|c|c|c|}
\hline $\begin{array}{c}Q^{2} \\
\left(\mathrm{GeV}^{2}\right)\end{array}$ & $x$ & $\begin{array}{r}\mathrm{d}^{2} \sigma_{c}^{\text {jet }} / \mathrm{d} x \mathrm{~d} Q^{2}\left(\mathrm{pb} / \mathrm{GeV}^{2}\right) \\
\text { stat. } \quad \text { syst. }\end{array}$ & $C^{\text {had }}$ & $C^{\mathrm{rad}}$ \\
\hline $5: \quad 20$ & $0.00008: 0.0002$ & $10700000 \pm 870000_{-600000}^{+740000}$ & 1.19 & 0.96 \\
\hline $5: \quad 20$ & $0.0002: 0.0003$ & $13500000 \pm 950000_{-730000}^{+890000}$ & 1.21 & 0.98 \\
\hline 20 & $0.0003: 0.0005$ & $8220000 \pm 470000_{-470000}^{+540000}$ & 1.23 & 0.98 \\
\hline $5: \quad 20$ & $0.0005: 0.003$ & $1620000 \pm 56000_{-87000}^{+100000}$ & 1.07 & 1.00 \\
\hline $20: \quad 60$ & $0.0003: 0.0005$ & $1570000 \pm 230000_{-120000}^{+140000}$ & 1.13 & 0.97 \\
\hline $20: \quad 60$ & $0.0005: 0.0012$ & $2600000 \pm 110000_{-120000}^{+140000}$ & 1.09 & 0.97 \\
\hline $20: \quad 60$ & $0.0012: 0.002$ & $1390000 \pm 68000 \stackrel{+73000}{+69000}$ & 1.05 & 0.98 \\
\hline $20: \quad 60$ & $: 0.0035$ & $650000 \pm 34000{ }_{-46000}^{+48000}$ & 1.01 & 0.99 \\
\hline $20: \quad 60$ & $0.0035: 0.01$ & $190000 \pm 13000 \stackrel{-13000}{+14000}^{+13}$ & 0.91 & 0.99 \\
\hline $60: 120$ & $0.0008: 0.0018$ & $251000 \pm 33000{ }_{-34000}^{+33000}$ & 1.07 & 0.97 \\
\hline $60: 120$ & $0.0018: 0.003$ & $283000 \pm 21000{ }_{-21000}^{+22000}$ & 1.03 & 0.99 \\
\hline $60: 120$ & $: 0.006$ & $136000 \pm 8100 \stackrel{-9700}{+10000}^{+10}$ & 1.01 & 0.98 \\
\hline $60: 120$ & $0.006 \quad: 0.04$ & $17100 \pm \quad 1600 \quad{ }_{-1200}^{+1400}$ & 0.93 & 0.93 \\
\hline $120: 400$ & $0.0016: 0.005$ & $110000 \pm \quad 7800 \quad \begin{array}{l}+5600 \\
5600\end{array}$ & 1.05 & 0.97 \\
\hline $120: 400$ & 0.005 & $34500 \pm \quad 2200 \quad{ }_{-1700}^{+1900}$ & 1.01 & 1.00 \\
\hline $120: 400$ & $0.016: 0.06$ & $5300 \pm \quad 1100 \quad{ }_{-800}^{+800}$ & 0.96 & 0.80 \\
\hline $400: 1000$ & $0.005: 0.02$ & $900 \quad \begin{array}{l}+900 \\
-850\end{array}$ & 1.02 & 0.88 \\
\hline $400: 1000$ & 0.02 & $540 \pm$ & 1.01 & 0.84 \\
\hline
\end{tabular}

Table 7. Double-differential cross sections for inclusive jet production in charm events as a function of $x$ for different ranges of $Q^{2}$. The cross sections are given for $5<Q^{2}<1000 \mathrm{GeV}^{2}, 0.02<y<0.7$, $E_{T}^{\text {jet }}>4.2 \mathrm{GeV}$ and $-1.6<\eta^{\text {jet }}<2.2$. The measurements are given together with their statistical and systematic uncertainties (not including the error on the integrated luminosity). Hadronisation and QED radiative corrections, $C^{\text {had }}$ and $C^{\text {rad }}$, respectively, are also shown. 


\begin{tabular}{|c|c|c|c|c|}
\hline \multirow{2}{*}{$\begin{array}{c}Q^{2} \\
\left(\mathrm{GeV}^{2}\right)\end{array}$} & \multirow[t]{2}{*}{$x$} & \multicolumn{3}{|c|}{$F_{2}^{b \bar{b}}$} \\
\hline & & stat. & syst. & extr. \\
\hline 6.5 & 0.00015 & $0.00431 \pm 0.00068$ & $\begin{array}{l}+0.00054 \\
-0.00048\end{array}$ & $\begin{array}{l}+0.00034 \\
{ }_{-0.00029}\end{array}$ \\
\hline 6.5 & 0.00028 & $0.00357 \pm 0.00052$ & $\begin{array}{l}+0.00036 \\
-0.00033\end{array}$ & $\begin{array}{l}+0.00029 \\
-0.00025\end{array}$ \\
\hline 12 & 0.00043 & $0.00632 \pm 0.00069$ & $\begin{array}{l}+0.00062 \\
-0.00052\end{array}$ & ${ }_{-0.00034}^{+0.00044}$ \\
\hline 12 & 0.00065 & $0.00438 \pm 0.00053$ & $\begin{array}{l}+0.00047 \\
-0.00043 \\
\end{array}$ & $\begin{array}{l}+0.00020 \\
-0.00012 \\
\end{array}$ \\
\hline 25 & 0.00043 & $0.0118 \pm 0.0035$ & $\begin{array}{l}+0.0016 \\
-0.0016\end{array}$ & $\begin{array}{l}+0.0009 \\
-0.0007\end{array}$ \\
\hline 25 & 0.00080 & $0.0105 \pm 0.0011$ & $\begin{array}{l}+0.0008 \\
-0.0007\end{array}$ & $\begin{array}{l}+0.0006 \\
-0.0005\end{array}$ \\
\hline 30 & 0.0016 & $0.0099 \pm 0.0012$ & $\begin{array}{l}+0.0009 \\
-0.0009\end{array}$ & ${ }_{-0.0004}^{+0.0004}$ \\
\hline 30 & 0.0025 & $0.0067 \pm 0.0012$ & $\begin{array}{l}+0.0009 \\
-0.0009\end{array}$ & ${ }_{-0.0003}^{+0.0002}$ \\
\hline 30 & 0.0045 & $0.0041 \pm 0.0023$ & $\begin{array}{l}+0.0011 \\
-0.0012 \\
\end{array}$ & $\begin{array}{l}+0.0001 \\
-0.0001 \\
\end{array}$ \\
\hline 80 & 0.0016 & $0.0364 \pm 0.0049$ & $\begin{array}{l}+0.0035 \\
-0.0034\end{array}$ & $\begin{array}{l}+0.0012 \\
-0.0012\end{array}$ \\
\hline 80 & 0.0025 & $0.0195 \pm 0.0025$ & $\begin{array}{l}+0.0017 \\
-0.0018\end{array}$ & $\begin{array}{l}+0.0005 \\
-0.0005\end{array}$ \\
\hline 80 & 0.0045 & $0.0110 \pm 0.0020$ & $\begin{array}{l}+0.0013 \\
-0.0013\end{array}$ & $\begin{array}{l}+0.0002 \\
-0.0003\end{array}$ \\
\hline 160 & 0.0035 & $0.0230 \pm 0.0032$ & $\begin{array}{l}+0.0016 \\
-0.0017\end{array}$ & $\begin{array}{l}+0.0005 \\
-0.0003\end{array}$ \\
\hline 160 & 0.0080 & $0.0176 \pm 0.0016$ & $\begin{array}{l}+0.0008 \\
-0.0009\end{array}$ & $\begin{array}{l}+0.0004 \\
-0.0003\end{array}$ \\
\hline 160 & 0.020 & $0.0078 \pm 0.0039$ & $\begin{array}{l}+0.0021 \\
-0.0022\end{array}$ & $\begin{array}{l}+0.0003 \\
-0.0002\end{array}$ \\
\hline 600 & 0.013 & $0.0154 \pm 0.0034$ & $\begin{array}{l}+0.0022 \\
-0.0020\end{array}$ & $\begin{array}{l}+0.0001 \\
-0.0002\end{array}$ \\
\hline 600 & 0.035 & $0.0088 \pm 0.0026$ & $\begin{array}{l}+0.0019 \\
-0.0020\end{array}$ & $\begin{array}{l}{ }_{-0.0001}^{+0.0003} \\
\end{array}$ \\
\hline
\end{tabular}

Table 8. The structure function $F_{2}^{b \bar{b}}$ as a function of $x$ for seven different values of $Q^{2}$. The first error is statistical, the second systematic (not including the error on the integrated luminosity) and the last is the extrapolation uncertainty. The horizontal lines correspond to the bins in $Q^{2}$ in table 6 


\begin{tabular}{|c|l|ccc|}
\hline $\begin{array}{c}Q^{2} \\
\left(\mathrm{GeV}^{2}\right)\end{array}$ & $x$ & \multicolumn{3}{|c|}{$F_{2}^{c \bar{c}}$} \\
\hline 6.5 & 0.00015 & $0.202 \pm 0.016$ & ${ }_{-0.011}^{+0.014}$ & ${ }_{-0.042}^{+0.046}$ \\
6.5 & 0.00028 & $0.189 \pm 0.013$ & ${ }_{-0.010}^{+0.013}$ & ${ }_{-0.039}^{+0.039}$ \\
12 & 0.00043 & $0.231 \pm 0.013$ & ${ }_{-0.013}^{+0.015}$ & ${ }_{-0.044}^{+0.039}$ \\
12 & 0.00065 & $0.224 \pm 0.008$ & ${ }_{-0.012}^{+0.014}$ & ${ }_{-0.028}^{+0.028}$ \\
\hline 25 & 0.00043 & $0.492 \pm 0.071$ & ${ }_{-0.036}^{+0.044}$ & ${ }_{-0.073}^{+0.085}$ \\
25 & 0.00080 & $0.418 \pm 0.018$ & ${ }_{-0.020}^{+0.022}$ & ${ }_{-0.034}^{+0.030}$ \\
30 & 0.0016 & $0.304 \pm 0.015$ & ${ }_{-0.015}^{+0.016}$ & ${ }_{-0.011}^{+0.014}$ \\
30 & 0.0025 & $0.235 \pm 0.012$ & ${ }_{-0.017}^{+0.018}$ & ${ }_{-0.008}^{+0.006}$ \\
30 & 0.0045 & $0.195 \pm 0.014$ & ${ }_{-0.014}^{+0.015}$ & ${ }_{-0.000}^{+0.010}$ \\
\hline 80 & 0.0016 & $0.385 \pm 0.051$ & ${ }_{-0.051}^{+0.051}$ & ${ }_{-0.010}^{+0.018}$ \\
80 & 0.0025 & $0.324 \pm 0.024$ & ${ }_{-0.024}^{+0.025}$ & ${ }_{-0.015}^{+0.001}$ \\
80 & 0.0045 & $0.244 \pm 0.015$ & ${ }_{-0.017}^{+0.018}$ & ${ }_{-0.004}^{+0.004}$ \\
80 & 0.0080 & $0.214 \pm 0.020$ & ${ }_{-0.015}^{+0.017}$ & ${ }_{-0.002}^{+0.000}$ \\
\hline 160 & 0.0035 & $0.450 \pm 0.032$ & ${ }_{-0.023}^{+0.030}$ & ${ }_{-0.007}^{+0.010}$ \\
160 & 0.0080 & $0.195 \pm 0.012$ & ${ }_{-0.010}^{+0.011}$ & ${ }_{-0.003}^{+0.003}$ \\
160 & 0.020 & $0.151 \pm 0.031$ & ${ }_{-0.022}^{+0.022}$ & ${ }_{-0.000}^{+0.002}$ \\
\hline 600 & 0.013 & $0.242 \pm 0.038$ & ${ }_{-0.036}^{+0.038}$ & ${ }_{-0.002}^{+0.006}$ \\
600 & 0.035 & $0.071 \pm 0.037$ & ${ }_{-0.021}^{+0.020}$ & ${ }_{-0.001}^{+0.002}$ \\
\hline
\end{tabular}

Table 9. The structure function $F_{2}^{c \bar{c}}$ as a function of $x$ for seven different values of $Q^{2}$. The first error is statistical, the second systematic (not including the error on the integrated luminosity) and the last is the extrapolation uncertainty. The horizontal lines correspond to the bins in $Q^{2}$ in table 7 . 


\begin{tabular}{|c|c|ccc|}
\hline $\begin{array}{c}Q^{2} \\
\left(\mathrm{GeV}^{2}\right)\end{array}$ & $x$ & \multicolumn{3}{|c|}{$\sigma_{\mathrm{r}}^{b \bar{b}}$} \\
\hline 6.5 & 0.00015 & $0.00429 \pm 0.00068$ & ${ }_{-0.00048}^{+0.00053}{ }_{-0.00029}^{+0.00034}$ \\
6.5 & 0.00028 & $0.00357 \pm 0.00051$ & ${ }_{-0.00033}^{+0.00036}$ & ${ }_{-0.00025}^{+0.00029}$ \\
12 & 0.00043 & $0.00631 \pm 0.00069$ & ${ }_{-0.00052}^{+0.00061}$ & ${ }_{-0.00034}^{+0.00043}$ \\
12 & 0.00065 & $0.00436 \pm 0.00052$ & ${ }_{-0.00042}^{+0.00046}$ & ${ }_{-0.00010}^{+0.00023}$ \\
\hline 25 & 0.00043 & $0.0116 \pm 0.0035$ & ${ }_{-0.0015}^{+0.0015}$ & ${ }_{-0.0006}^{+0.0009}$ \\
25 & 0.00080 & $0.0104 \pm 0.0011$ & ${ }_{-0.0007}^{+0.0008}$ & ${ }_{-0.0005}^{+0.0006}$ \\
30 & 0.0016 & $0.0099 \pm 0.0012$ & ${ }_{-0.0009}^{+0.0009}$ & ${ }_{-0.0005}^{+0.0004}$ \\
30 & 0.0025 & $0.0067 \pm 0.0012$ & ${ }_{-0.0009}^{+0.0009}$ & ${ }_{-0.0002}^{+0.0002}$ \\
30 & 0.0045 & $0.0041 \pm 0.0023$ & ${ }_{-0.0012}^{+0.0011}$ & ${ }_{-0.0003}^{+0.0001}$ \\
\hline 80 & 0.0016 & $0.0354 \pm 0.0047$ & ${ }_{-0.0033}^{+0.0034}$ & ${ }_{-0.0012}^{+0.0001}$ \\
80 & 0.0025 & $0.0194 \pm 0.0025$ & ${ }_{-0.0018}^{+0.0017}$ & ${ }_{-0.0005}^{+0.0005}$ \\
80 & 0.0045 & $0.0109 \pm 0.0020$ & ${ }_{-0.0013}^{+0.0012}$ & ${ }_{-0.0003}^{+0.0003}$ \\
\hline 160 & 0.0035 & $0.0223 \pm 0.0031$ & ${ }_{-0.0017}^{+0.0015}$ & ${ }_{-0.0003}^{+0.0005}$ \\
160 & 0.0080 & $0.0176 \pm 0.0016$ & ${ }_{-0.0009}^{+0.0008}$ & ${ }_{-0.0004}^{+0.0004}$ \\
160 & 0.020 & $0.0078 \pm 0.0039$ & ${ }_{-0.0022}^{+0.0021}$ & ${ }_{-0.0001}^{+0.0002}$ \\
\hline 600 & 0.013 & $0.0149 \pm 0.0032$ & ${ }_{-0.0019}^{+0.0021}$ & ${ }_{-0.0002}^{+0.0001}$ \\
600 & 0.035 & $0.0088 \pm 0.0026$ & ${ }_{-0.0020}^{+0.0019}$ & ${ }_{-0.0001}^{+0.0003}$ \\
\hline
\end{tabular}

Table 10. Reduced beauty cross sections, $\sigma_{\mathrm{r}}^{b \bar{b}}$, as a function of $x$ for seven different values of $Q^{2}$. For more details, see the caption of table 8 . 


\begin{tabular}{|c|l|ccc|}
\hline $\begin{array}{c}Q^{2} \\
\left(\mathrm{GeV}^{2}\right)\end{array}$ & $x$ & \multicolumn{3}{|c|}{$\sigma_{\mathrm{r}}^{c \bar{c}}$} \\
\hline 6.5 & 0.00015 & $0.201 \pm 0.016$ & ${ }_{-0.011}^{+0.014}$ & ${ }_{-0.042}^{+0.041}$ \\
6.5 & 0.00028 & $0.188 \pm 0.013$ & ${ }_{-0.010}^{+0.012}$ & ${ }_{-0.042}^{+0.040}$ \\
12 & 0.00043 & $0.230 \pm 0.013$ & ${ }_{-0.013}^{+0.015}$ & ${ }_{-0.047}^{+0.037}$ \\
12 & 0.00065 & $0.224 \pm 0.008$ & ${ }_{-0.012}^{+0.014}$ & ${ }_{-0.034}^{+0.028}$ \\
\hline 25 & 0.00043 & $0.465 \pm 0.067$ & ${ }_{-0.034}^{+0.042}$ & ${ }_{-0.067}^{+0.081}$ \\
25 & 0.00080 & $0.413 \pm 0.018$ & ${ }_{-0.019}^{+0.022}$ & ${ }_{-0.035}^{+0.026}$ \\
30 & 0.0016 & $0.304 \pm 0.015$ & ${ }_{-0.015}^{+0.016}$ & ${ }_{-0.013}^{+0.014}$ \\
30 & 0.0025 & $0.234 \pm 0.012$ & ${ }_{-0.017}^{+0.017}$ & ${ }_{-0.008}^{+0.006}$ \\
30 & 0.0045 & $0.194 \pm 0.014$ & ${ }_{-0.014}^{+0.015}$ & ${ }_{-0.000}^{+0.011}$ \\
\hline 80 & 0.0016 & $0.369 \pm 0.049$ & ${ }_{-0.049}^{+0.049}$ & ${ }_{-0.010}^{+0.018}$ \\
80 & 0.0025 & $0.319 \pm 0.024$ & ${ }_{-0.024}^{+0.025}$ & ${ }_{-0.015}^{+0.002}$ \\
80 & 0.0045 & $0.243 \pm 0.014$ & ${ }_{-0.017}^{+0.018}$ & ${ }_{-0.005}^{+0.004}$ \\
80 & 0.0080 & $0.213 \pm 0.020$ & ${ }_{-0.015}^{+0.017}$ & ${ }_{-0.003}^{+0.000}$ \\
\hline 160 & 0.0035 & $0.436 \pm 0.031$ & ${ }_{-0.022}^{+0.029}$ & ${ }_{-0.007}^{+0.009}$ \\
160 & 0.0080 & $0.194 \pm 0.012$ & ${ }_{-0.010}^{+0.011}$ & ${ }_{-0.005}^{+0.003}$ \\
160 & 0.020 & $0.151 \pm 0.031$ & ${ }_{-0.022}^{+0.022}$ & ${ }_{-0.000}^{+0.001}$ \\
\hline 600 & 0.013 & $0.235 \pm 0.037$ & ${ }_{-0.034}^{+0.037}$ & ${ }_{-0.002}^{+0.006}$ \\
600 & 0.035 & $0.070 \pm 0.037$ & ${ }_{-0.020}^{+0.020}$ & ${ }_{-0.001}^{+0.002}$ \\
\hline
\end{tabular}

Table 11. Reduced charm cross section, $\sigma_{\mathrm{r}}^{c \bar{c}}$, as a function of $x$ for seven different values of $Q^{2}$. For more details, see the caption of table 9. 


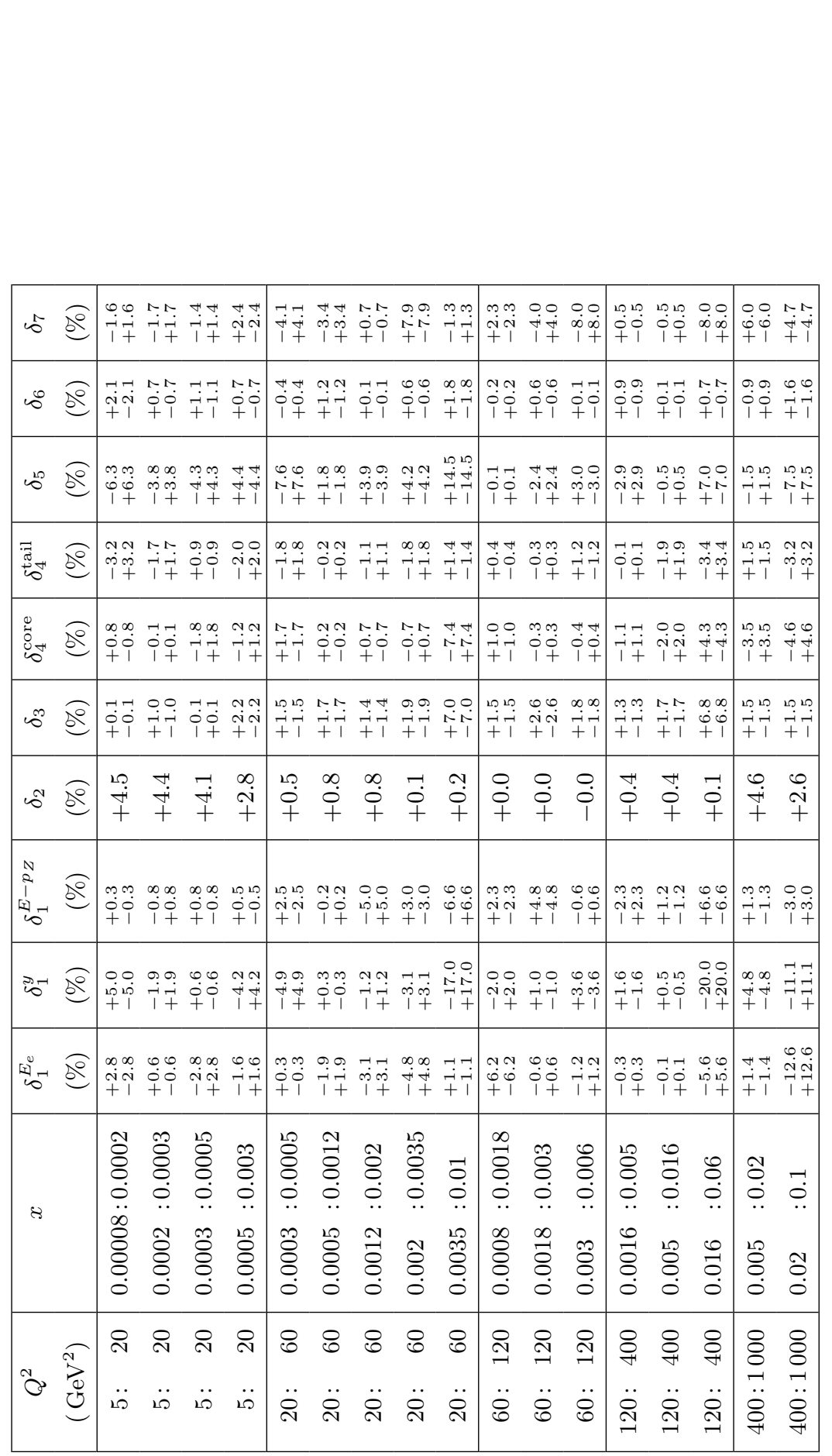




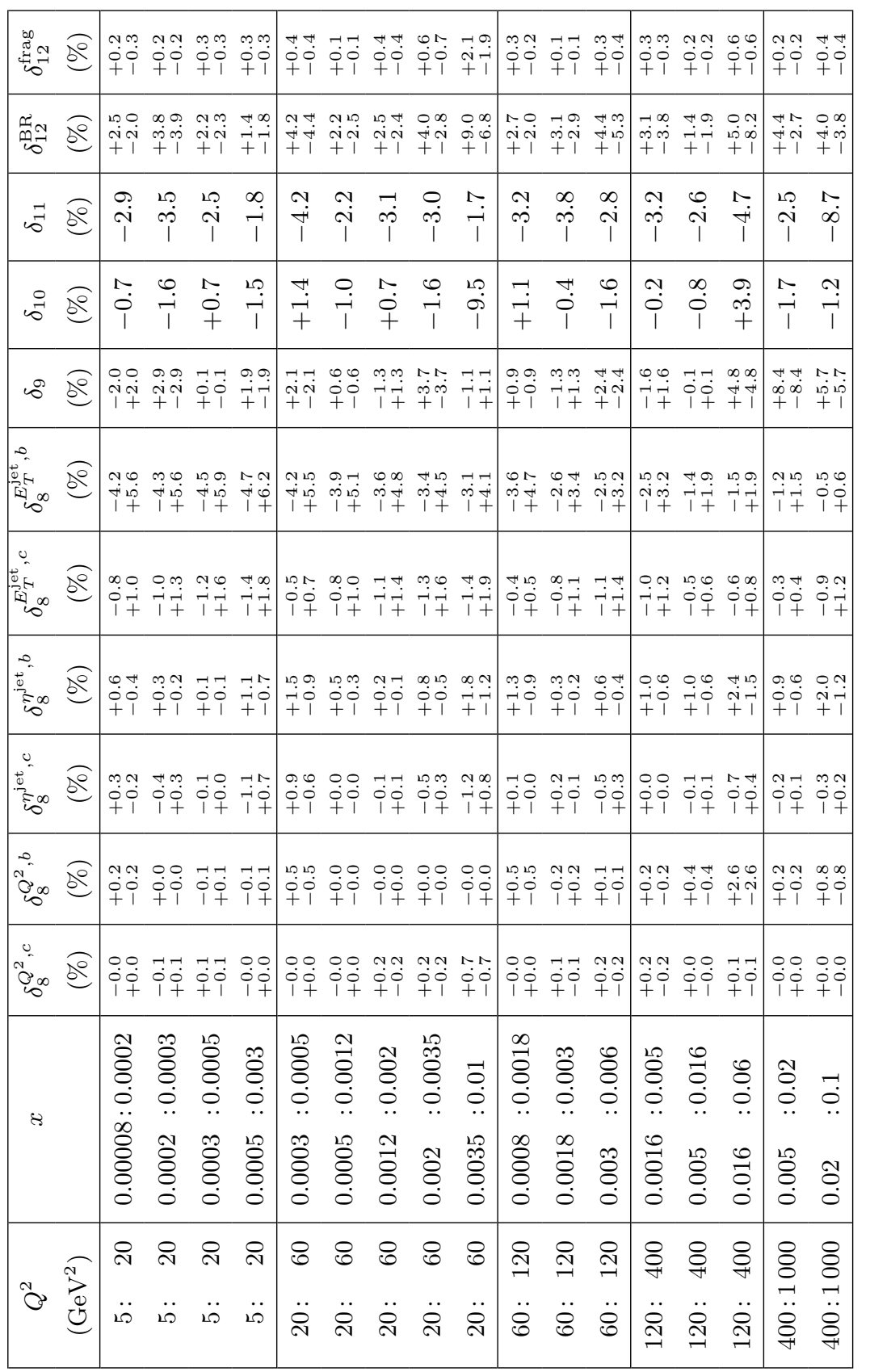




\begin{tabular}{|c|c|c|c|c|c|}
\hline 5 & 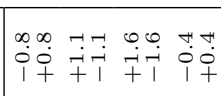 & 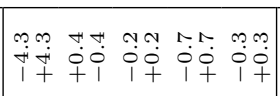 & 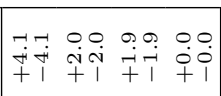 & 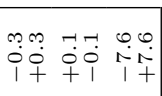 & 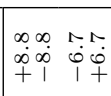 \\
\hline$\therefore \widehat{8}$ & 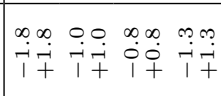 & $\stackrel{\circ}{\circ}+\dot{i}$ & $\begin{array}{l}0 \\
\dot{0} \\
i \\
1+\end{array}$ & $\dot{0} 0$ & 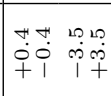 \\
\hline 10 & 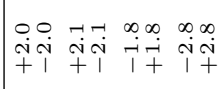 & 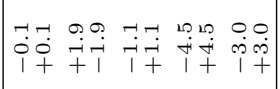 & 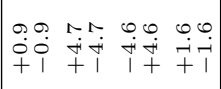 & 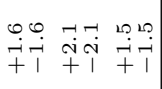 & 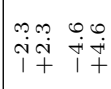 \\
\hline$\widehat{\bar{J}}$ & $\begin{array}{ll}0 & 0 \\
0 & 0 \\
+ & 0 \\
1 & 1\end{array}$ & 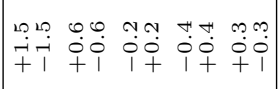 & & & \\
\hline 总 & 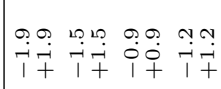 & $\mid \begin{array}{ll}\circ & 0 \\
\text { ini } \\
1+\end{array}$ & & तनت & \\
\hline$\infty \widehat{\infty}$ & 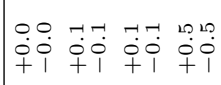 & 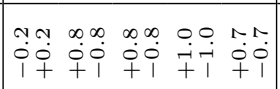 & تनें & $\overrightarrow{7+7}$ & $\begin{array}{lll}\infty & \infty \\
0 & \infty \\
+1 & 0\end{array}$ \\
\hline s $\widehat{S}$ & 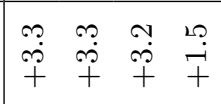 & 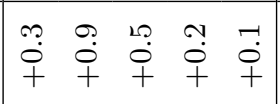 & $\begin{array}{|cccc|} & 0 & 0 & 0 \\
\dot{0} & \dot{0} & \dot{0} & \dot{0} \\
+ & + & + & +\end{array}$ & 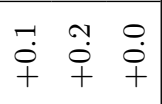 & $\begin{array}{ll}\stackrel{P}{+} & \Re \\
\stackrel{+}{+} & +\end{array}$ \\
\hline 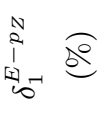 & 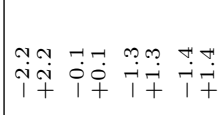 & & 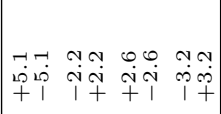 & $\begin{array}{l}\text { ag } \\
\dot{0}+ \\
+1\end{array}$ & 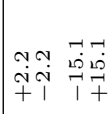 \\
\hline 烦 $\widehat{\widehat{S}}$ & 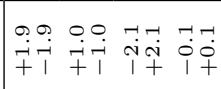 & & & & 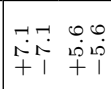 \\
\hline 幽- & 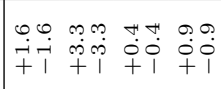 & 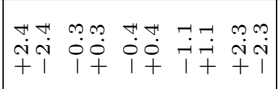 & & 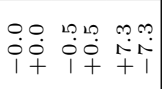 & 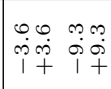 \\
\hline 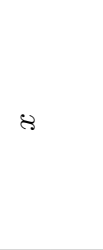 & 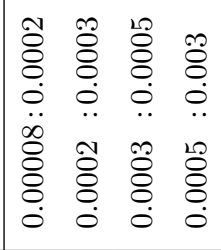 & 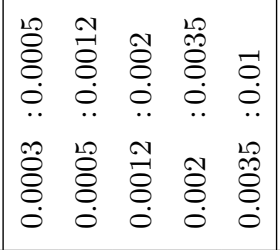 & 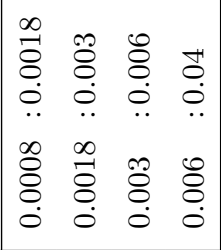 & $\begin{array}{ccc}20 & 0 & 0 \\
0 & 0 & 0 \\
0 & 0 & 0 \\
0 & 0 & 0 \\
0 & . & . \\
0 & & \\
0 & 2 & 0 \\
0 & 0 & 0 \\
0 & 0 & 0 \\
0 & 0 & 0\end{array}$ & 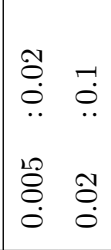 \\
\hline$\approx$ 染 & $\mid \begin{array}{llll}\text { จ } & \text { จ } & \text { จ } & \text { จ } \\
\ddot{b} & \ddot{b} & \ddot{b} & \ddot{b}\end{array}$ & $\left|\begin{array}{ccccc|}\ddot{0} & \& & 8 & \& & 8 \\
\ddot{\sim} & \ddot{\sim} & \ddot{\sim} & \ddot{\sim} & \ddot{\sim}\end{array}\right|$ & 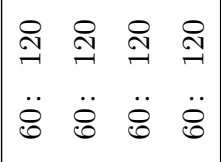 & 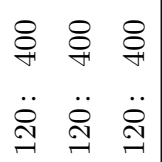 & 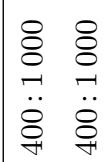 \\
\hline
\end{tabular}




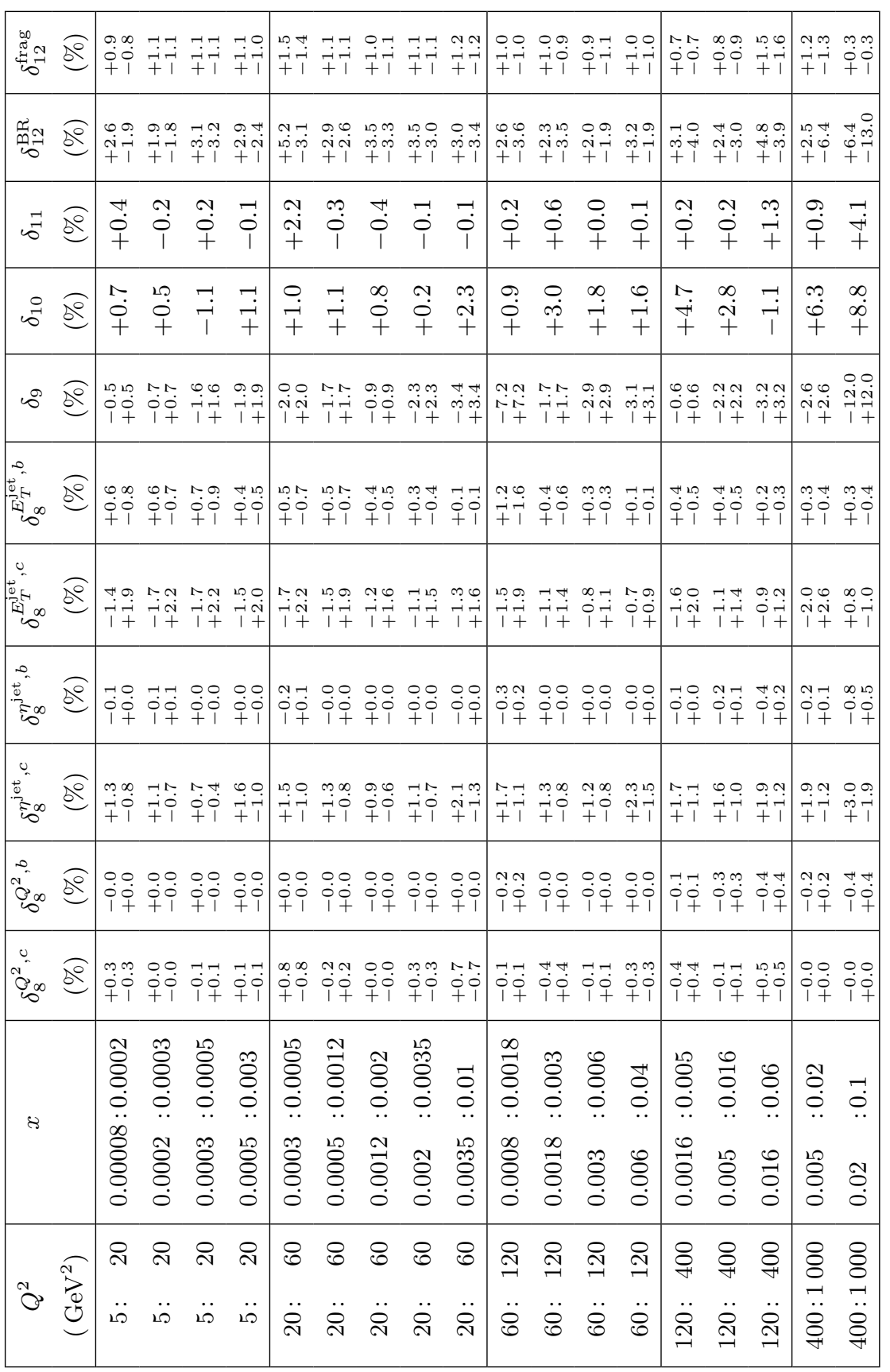




\begin{tabular}{|c|l|c|c|c|c|c|c|}
\hline $\begin{array}{c}Q^{2} \\
\left(\mathrm{GeV}^{2}\right)\end{array}$ & $x$ & $\begin{array}{c}\delta_{m_{b}}^{-} \\
(\%)\end{array}$ & $\begin{array}{c}\delta_{m_{b}}^{+} \\
(\%)\end{array}$ & $\begin{array}{c}\delta_{\mu_{R}, \mu_{F}}^{-} \\
(\%)\end{array}$ & $\begin{array}{c}\delta_{\mu_{R}, \mu_{F}}^{+} \\
(\%)\end{array}$ & $\begin{array}{c}\delta_{\alpha_{s}}^{-} \\
(\%)\end{array}$ & $\begin{array}{c}\delta_{\alpha_{s}}^{+} \\
(\%)\end{array}$ \\
\hline 6.5 & 0.00015 & +7.4 & -5.9 & -3.3 & +2.6 & +0.7 & -0.3 \\
6.5 & 0.00028 & +8.1 & -6.8 & -1.5 & +0.5 & +0.1 & -0.4 \\
12 & 0.00043 & +6.9 & -5.2 & -1.4 & +1.0 & +0.3 & -0.2 \\
12 & 0.00065 & +4.2 & -2.7 & -0.8 & +1.7 & +0.3 & -0.0 \\
\hline 25 & 0.00043 & +7.2 & -5.1 & -2.2 & +2.3 & +0.8 & -0.3 \\
25 & 0.00080 & +5.8 & -4.6 & -0.7 & +0.4 & +0.2 & -0.0 \\
30 & 0.0016 & +4.3 & -4.1 & +0.3 & -1.2 & -0.3 & -0.4 \\
30 & 0.0025 & +3.1 & -3.3 & +1.1 & -1.7 & -0.3 & -0.6 \\
30 & 0.0045 & +1.6 & -0.8 & +2.6 & -0.9 & -1.0 & -0.2 \\
\hline 80 & 0.0016 & +3.2 & -3.1 & -1.5 & -0.2 & +0.2 & -0.1 \\
80 & 0.0025 & +2.6 & -2.3 & -0.1 & -0.7 & +0.3 & +0.2 \\
80 & 0.0045 & +1.9 & -2.2 & +0.8 & -1.6 & -0.5 & -0.0 \\
\hline 160 & 0.0035 & +2.2 & -1.5 & -0.2 & -0.1 & +0.2 & -0.3 \\
160 & 0.0080 & +2.1 & -1.6 & +1.3 & -1.1 & +0.1 & +0.2 \\
160 & 0.020 & +0.8 & +0.0 & +3.1 & -1.9 & +0.9 & -0.2 \\
\hline 600 & 0.013 & +0.8 & -1.3 & +0.1 & -0.4 & +0.2 & -0.5 \\
600 & 0.035 & +1.1 & +0.4 & +2.6 & -1.2 & +0.3 & +0.7 \\
\hline
\end{tabular}

Table 16. Extrapolation uncertainties on the structure function $F_{2}^{b \bar{b}}$ due to the variations of the beauty-quark mass, $m_{b}$, factorisation and renormalisation scales, $\mu_{F}$ and $\mu_{R}$, and the strong coupling constant, $\alpha_{s}$. The plus (minus) superscript indicates the upward (downward) variation of the corresponding parameter. See section 9 for more details. 


\begin{tabular}{|c|c|c|c|c|c|c|c|}
\hline $\begin{array}{c}Q^{2} \\
\left(\mathrm{GeV}^{2}\right)\end{array}$ & $x$ & $\begin{array}{c}\delta_{m_{c}}^{-} \\
(\%)\end{array}$ & $\begin{array}{c}\delta_{m_{c}}^{+} \\
(\%)\end{array}$ & $\begin{array}{c}\delta_{\mu_{R}, \mu_{F}}^{-} \\
(\%)\end{array}$ & $\begin{array}{c}\delta_{\mu_{R}, \mu_{F}}^{+} \\
(\%)\end{array}$ & $\begin{array}{c}\delta_{\alpha_{s}}^{-} \\
(\%)\end{array}$ & $\begin{array}{c}\delta_{\alpha_{s}}^{+} \\
(\%)\end{array}$ \\
\hline 6.5 & 0.00015 & +9.9 & -6.8 & -19.6 & +20.2 & +4.8 & -1.0 \\
6.5 & 0.00028 & +9.2 & -9.6 & -17.7 & +18.0 & +4.3 & -3.0 \\
12 & 0.00043 & +7.4 & -6.9 & -17.5 & +14.8 & +3.2 & -3.4 \\
12 & 0.00065 & +6.1 & -4.6 & -11.4 & +10.8 & +2.5 & -2.8 \\
\hline 25 & 0.00043 & +8.1 & -5.1 & -13.9 & +14.9 & +3.7 & -0.8 \\
25 & 0.00080 & +5.7 & -4.6 & -6.7 & +4.1 & +0.7 & -0.8 \\
30 & 0.0016 & +4.5 & -3.3 & -1.3 & -0.5 & -0.3 & +0.3 \\
30 & 0.0025 & +2.4 & -3.4 & +0.2 & -1.0 & -0.4 & -0.3 \\
30 & 0.0045 & +3.5 & +0.2 & +3.5 & +0.2 & +1.6 & +1.5 \\
\hline 80 & 0.0016 & +3.6 & -1.8 & -1.7 & +2.6 & +0.6 & +1.0 \\
80 & 0.0025 & +0.4 & -3.1 & -2.1 & -1.8 & -1.4 & -1.0 \\
80 & 0.0045 & +1.5 & -1.4 & -0.2 & -0.9 & -0.2 & -0.4 \\
80 & 0.0080 & -0.8 & -0.2 & -0.4 & -0.3 & -0.4 & -0.3 \\
\hline 160 & 0.0035 & +2.1 & -1.2 & -0.7 & +0.6 & -0.3 & -0.7 \\
160 & 0.0080 & +1.2 & -1.4 & +0.7 & +0.3 & -0.1 & -0.5 \\
160 & 0.020 & -0.2 & +0.1 & +0.3 & +0.8 & -0.0 & +1.1 \\
\hline 600 & 0.013 & +2.0 & -0.5 & -0.8 & +0.5 & +1.0 & +0.6 \\
600 & 0.035 & +1.6 & +0.3 & +1.7 & -1.2 & +2.2 & +1.2 \\
\hline
\end{tabular}

Table 17. Extrapolation uncertainties on the structure function $F_{2}^{c \bar{c}}$ due to the variations of the charm-quark mass, $m_{c}$, factorisation and renormalisation scales, $\mu_{F}$ and $\mu_{R}$, and the strong coupling constant, $\alpha_{s}$. The plus (minus) superscript indicates the upward (downward) variation of the corresponding parameter. See section 9 for more details. 


\begin{tabular}{|c|l|c|c|c|c|c|c|}
\hline $\begin{array}{c}Q^{2} \\
\left(\mathrm{GeV}^{2}\right)\end{array}$ & $x$ & $\begin{array}{c}\delta_{m_{b}}^{-} \\
(\%)\end{array}$ & $\begin{array}{c}\delta_{m_{b}}^{+} \\
(\%)\end{array}$ & $\begin{array}{c}\delta_{\mu_{R}, \mu_{F}}^{-} \\
(\%)\end{array}$ & $\begin{array}{c}\delta_{\mu_{R}, \mu_{F}}^{+} \\
(\%)\end{array}$ & $\begin{array}{c}\delta_{\alpha_{s}}^{-} \\
(\%)\end{array}$ & $\begin{array}{c}\delta_{\alpha_{s}}^{+} \\
(\%)\end{array}$ \\
\hline 6.5 & 0.00015 & +7.3 & -6.0 & -3.2 & +2.6 & +0.7 & -0.3 \\
6.5 & 0.00028 & +8.1 & -6.8 & -1.3 & +0.6 & +0.3 & -0.3 \\
12 & 0.00043 & +6.8 & -5.2 & -1.4 & +0.9 & +0.2 & -0.3 \\
12 & 0.00065 & +4.7 & -2.2 & -0.4 & +2.1 & +0.8 & +0.4 \\
\hline 25 & 0.00043 & +7.1 & -4.9 & -2.6 & +2.4 & +0.8 & -0.3 \\
25 & 0.00080 & +5.7 & -4.8 & -0.7 & +0.5 & +0.2 & -0.1 \\
30 & 0.0016 & +4.0 & -4.4 & +0.3 & -1.7 & -0.7 & -0.6 \\
30 & 0.0025 & +3.2 & -3.0 & +1.3 & -1.4 & +0.1 & -0.2 \\
30 & 0.0045 & +1.7 & -3.2 & +0.5 & -4.2 & -2.4 & -2.2 \\
\hline 80 & 0.0016 & +3.1 & -3.0 & -1.3 & -0.0 & +0.1 & -0.1 \\
80 & 0.0025 & +2.5 & -2.4 & -0.1 & -0.8 & +0.2 & +0.2 \\
80 & 0.0045 & +2.2 & -2.0 & +1.0 & -1.2 & +0.0 & +0.2 \\
\hline 160 & 0.0035 & +2.2 & -1.4 & -0.2 & -0.1 & +0.3 & -0.3 \\
160 & 0.0080 & +1.9 & -1.7 & +1.1 & -1.2 & -0.0 & +0.1 \\
160 & 0.020 & +0.5 & -0.2 & +2.9 & -1.8 & +0.5 & -0.0 \\
\hline 600 & 0.013 & +0.7 & -1.3 & +0.1 & -0.4 & +0.3 & -0.5 \\
600 & 0.035 & +1.0 & +0.4 & +2.7 & -1.4 & +0.2 & +0.8 \\
\hline
\end{tabular}

Table 18. Extrapolation uncertainties on the reduced beauty cross section, $\sigma_{\mathrm{r}}^{b \bar{b}}$, due to the variations of the beauty-quark mass, $m_{b}$, factorisation and renormalisation scales, $\mu_{F}$ and $\mu_{R}$, and the strong coupling constant, $\alpha_{s}$. The plus (minus) superscript indicates the upward (downward) variation of the corresponding parameter. See section 9 for more details. 


\begin{tabular}{|c|c|c|c|c|c|c|c|}
\hline $\begin{array}{c}Q^{2} \\
\left(\mathrm{GeV}^{2}\right)\end{array}$ & $x$ & $\begin{array}{c}\delta_{m_{c}}^{-} \\
(\%)\end{array}$ & $\begin{array}{c}\delta_{m_{c}}^{+} \\
(\%)\end{array}$ & $\begin{array}{c}\delta_{\mu_{R}, \mu_{F}}^{-} \\
(\%)\end{array}$ & $\begin{array}{c}\delta_{\mu_{R}, \mu_{F}}^{+} \\
(\%)\end{array}$ & $\begin{array}{c}\delta_{\alpha_{s}}^{-} \\
(\%)\end{array}$ & $\begin{array}{c}\delta_{\alpha_{s}}^{+} \\
(\%)\end{array}$ \\
\hline 6.5 & 0.00015 & +8.3 & -7.7 & -19.1 & +18.2 & +3.5 & -2.3 \\
6.5 & 0.00028 & +9.3 & -9.0 & -20.3 & +18.7 & +3.9 & -2.4 \\
12 & 0.00043 & +7.0 & -7.5 & -18.6 & +14.2 & +2.1 & -3.3 \\
12 & 0.00065 & +5.7 & -4.8 & -14.1 & +11.3 & +0.9 & -2.8 \\
\hline 25 & 0.00043 & +7.8 & -4.3 & -13.9 & +15.1 & +3.7 & -0.4 \\
25 & 0.00080 & +5.0 & -4.8 & -6.9 & +4.0 & +0.8 & -0.8 \\
30 & 0.0016 & +4.5 & -3.9 & -1.2 & -0.8 & -0.7 & +0.3 \\
30 & 0.0025 & +2.4 & -3.4 & +0.5 & -1.0 & +0.1 & +0.2 \\
30 & 0.0045 & +3.0 & +0.4 & +4.0 & +0.6 & +1.9 & +1.9 \\
\hline 80 & 0.0016 & +3.7 & -1.8 & -2.0 & +2.8 & +0.6 & +1.0 \\
80 & 0.0025 & +0.6 & -3.2 & -2.1 & -1.8 & -1.5 & -0.9 \\
80 & 0.0045 & +1.5 & -1.8 & -0.1 & -0.9 & -0.3 & -0.4 \\
80 & 0.0080 & -1.0 & -0.2 & -0.7 & -0.7 & -0.4 & -0.2 \\
\hline 160 & 0.0035 & +2.1 & -1.3 & -0.6 & +0.6 & -0.4 & -0.7 \\
160 & 0.0080 & +1.3 & -2.2 & +0.6 & -0.3 & -0.5 & -0.7 \\
160 & 0.020 & -0.2 & +0.3 & +0.5 & -0.2 & +0.1 & +0.7 \\
\hline 600 & 0.013 & +2.3 & -0.5 & -0.7 & +0.5 & +1.1 & +0.7 \\
600 & 0.035 & +1.6 & +0.3 & +1.7 & -1.2 & +1.6 & +1.4 \\
\hline
\end{tabular}

Table 19. Extrapolation uncertainties on the reduced charm cross section, $\sigma_{\mathrm{r}}^{c \bar{c}}$, due to the variations of the charm-quark mass, $m_{c}$, factorisation and renormalisation scales, $\mu_{F}$ and $\mu_{R}$, and the strong coupling constant, $\alpha_{s}$. The plus (minus) superscript indicates the upward (downward) variation of the corresponding parameter. See section 9 for more details. 


\begin{tabular}{|l|c|c|}
\hline Parameter & Variation & $\begin{array}{c}\text { Uncertainty } \\
(\mathrm{GeV})\end{array}$ \\
\hline \multicolumn{3}{|c|}{ Fit uncertainty } \\
\hline Total & $\Delta \chi^{2}=1$ & ${ }_{-0.14}^{+0.14}$ \\
\hline \multicolumn{3}{|c|}{ Model uncertainty } \\
\hline$f_{s}$ & $0.31_{-0.08}^{+0.07}$ & ${ }_{-0.00}^{+0.00}$ \\
$Q_{\text {min }}^{2}$ & $3.5 \rightarrow 5.0 \mathrm{GeV}^{2}$ & ${ }_{-0.00}^{+0.00}$ \\
$Q_{0}^{2}$ & $1.4 \rightarrow 1.9 \mathrm{GeV}^{2}$ & ${ }_{-0.01}^{+0.01}$ \\
$\delta m_{\text {ext }}$ & see text & ${ }_{-0.07}$ \\
\hline Total & \multicolumn{2}{|c|}{${ }_{-0.07}^{+0.01}$} \\
\hline \multicolumn{3}{|c|}{ PDF parameterisation uncertainty } \\
\hline$D_{u_{v}}$ & free in fit & ${ }_{+0.03}$ \\
$D_{\bar{D}}$ & free in fit & ${ }_{+0.03}$ \\
$D_{\bar{U}}$ & free in fit & ${ }_{+0.02}^{+0.05}$ \\
\hline Total & \multicolumn{3}{|c|}{ Theory uncertainty } \\
\hline \multicolumn{3}{|c|}{} \\
\hline$m_{c}\left(m_{c}\right)$ & $(1.26 \pm 0.06) \mathrm{GeV}$ & ${ }_{-0.02}^{+0.02}$ \\
$\alpha_{s}$ & $0.105 \pm 0.002$ & ${ }_{-0.02}^{+0.02}$ \\
$\mu$ & $\times 2, \times 1 / 2$ & ${ }_{-0.04}^{+0.07}$ \\
\hline Total & ${ }_{-0.05}^{+0.08}$ \\
\hline
\end{tabular}

Table 20. List of uncertainties for the beauty-quark mass determination. A description of the uncertainties not explicitly mentioned in the text is given elsewhere [32].

Open Access. This article is distributed under the terms of the Creative Commons Attribution License (CC-BY 4.0), which permits any use, distribution and reproduction in any medium, provided the original author(s) and source are credited.

\section{References}

[1] B.W. Harris and J. Smith, Heavy quark correlations in deep inelastic electroproduction, Nucl. Phys. B 452 (1995) 109 [hep-ph/9503484] [INSPIRE].

[2] B.W. Harris and J. Smith, Invariant mass distributions for heavy quark-anti-quark pairs in deep inelastic electroproduction, Phys. Lett. B 353 (1995) 535 [Erratum ibid. B 359 (1995) 423] [hep-ph/9502312] [INSPIRE].

[3] E. Laenen, S. Riemersma, J. Smith and W.L. van Neerven, Complete $O\left(\alpha_{s}\right)$ corrections to heavy flavor structure functions in electroproduction, Nucl. Phys. B 392 (1993) 162 [INSPIRE]. 
[4] E. Laenen, S. Riemersma, J. Smith and W.L. van Neerven, $O\left(\alpha_{s}\right)$ corrections to heavy flavor inclusive distributions in electroproduction, Nucl. Phys. B 392 (1993) 229 [INSPIRE].

[5] H1 collaboration, C. Adloff et al., Inclusive $D^{0}$ and $D^{* \pm}$ production in deep inelastic ep scattering at HERA, Z. Phys. C 72 (1996) 593 [hep-ex/9607012] [INSPIRE].

[6] H1 collaboration, C. Adloff et al., Measurement of $D^{*}$ meson cross-sections at HERA and determination of the gluon density in the proton using NLO QCD, Nucl. Phys. B $\mathbf{5 4 5}$ (1999) 21 [hep-ex/9812023] [INSPIRE].

[7] H1 collaboration, C. Adloff et al., Measurement of $D^{* \pm}$ meson production and $F_{2}^{c}$ in deep inelastic scattering at HERA, Phys. Lett. B 528 (2002) 199 [hep-ex/0108039] [INSPIRE].

[8] H1 collaboration, A. Aktas et al., Inclusive production of $D^{+}, D^{0}, D_{s}^{+}$and $D^{*+}$ mesons in deep inelastic scattering at HERA, Eur. Phys. J. C 38 (2005) 447 [hep-ex/0408149] [INSPIRE].

[9] H1 collaboration, A. Aktas et al., Measurement of $F_{2}(c \bar{c})$ and $F_{2}(b \bar{b})$ at high $Q^{2}$ using the $H 1$ vertex detector at HERA, Eur. Phys. J. C 40 (2005) 349 [hep-ex/0411046] [InSPIRE].

[10] H1 collaboration, A. Aktas et al., Measurement of beauty production at HERA using events with muons and jets, Eur. Phys. J. C 41 (2005) 453 [hep-ex/0502010] [INSPIRE].

[11] H1 collaboration, A. Aktas et al., Measurement of $F_{2}^{c \bar{c}}$ and $F_{2}^{b \bar{b}}$ at low $Q^{2}$ and $x$ using the H1 vertex detector at HERA, Eur. Phys. J. C 45 (2006) 23 [hep-ex/0507081] [InSPIRE].

[12] H1 collaboration, A. Aktas et al., Production of $D^{* \pm}$ mesons with dijets in deep-inelastic scattering at HERA, Eur. Phys. J. C 51 (2007) 271 [hep-ex/0701023] [INSPIRE].

[13] H1 collaboration, F.D. Aaron et al., Measurement of the $D^{* \pm}$ meson production cross section and $F_{2}^{c \bar{c}}$, at high $Q^{2}$, in ep scattering at HERA, Phys. Lett. B 686 (2010) 91 [arXiv:0911.3989] [INSPIRE].

[14] H1 collaboration, F.D. Aaron et al., Measurement of the charm and beauty structure functions using the H1 vertex detector at HERA, Eur. Phys. J. C 65 (2010) 89 [arXiv:0907.2643] [INSPIRE].

[15] H1 collaboration, F.D. Aaron et al., Measurement of charm and beauty jets in deep inelastic scattering at HERA, Eur. Phys. J. C 71 (2011) 1509 [arXiv:1008.1731] [INSPIRE].

[16] H1 collaboration, F.D. Aaron et al., Measurement of $D^{* \pm}$ meson production and determination of $F_{2}^{c \bar{c}}$ at low $Q^{2}$ in deep-inelastic scattering at HERA, Eur. Phys. J. C 71 (2011) 1769 [Erratum ibid. C 72 (2012) 2252] [arXiv:1106.1028] [INSPIRE].

[17] ZEUS collaboration, J. Breitweg et al., $D^{*}$ production in deep inelastic scattering at HERA, Phys. Lett. B 407 (1997) 402 [hep-ex/9706009] [InSPIRE].

[18] ZEUS collaboration, J. Breitweg et al., Measurement of $D^{* \pm}$ production and the charm contribution to $F_{2}$ in deep inelastic scattering at HERA, Eur. Phys. J. C 12 (2000) 35 [hep-ex/9908012] [INSPIRE].

[19] ZEUS collaboration, S. Chekanov et al., Measurement of $D^{* \pm}$ production in deep inelastic $e^{ \pm} p$ scattering at HERA, Phys. Rev. D 69 (2004) 012004 [hep-ex/0308068] [INSPIRE].

[20] ZEUS collaboration, S. Chekanov et al., Measurement of beauty production in deep inelastic scattering at HERA, Phys. Lett. B 599 (2004) 173 [hep-ex/0405069] [INSPIRE].

[21] ZEUS collaboration, S. Chekanov et al., Measurement of open beauty production at HERA in the $D^{*}$ muon final state, Eur. Phys. J. C 50 (2007) 299 [hep-ex/0609050] [InSPIRE]. 
[22] ZEUS collaboration, S. Chekanov et al., Measurement of $D^{* \pm}$ meson production in $e^{ \pm} p$ scattering at low $Q^{2}$, Phys. Lett. B 649 (2007) 111 [hep-ex/0702034] [INSPIRE].

[23] ZEUS collaboration, S. Chekanov et al., Measurement of D mesons production in deep inelastic scattering at HERA, JHEP 07 (2007) 074 [arXiv:0704.3562] [INSPIRE].

[24] ZEUS collaboration, S. Chekanov et al., Measurement of beauty production from dimuon events at HERA, JHEP 02 (2009) 032 [arXiv:0811.0894] [INSPIRE].

[25] ZEUS collaboration, S. Chekanov et al., Measurement of $D^{ \pm}$and $D^{0}$ production in deep inelastic scattering using a lifetime tag at HERA, Eur. Phys. J. C 63 (2009) 171 [arXiv:0812.3775] [INSPIRE].

[26] ZEUS collaboration, S. Chekanov et al., Measurement of charm and beauty production in deep inelastic ep scattering from decays into muons at HERA, Eur. Phys. J. C 65 (2010) 65 [arXiv: 0904.3487] [INSPIRE].

[27] ZEUS collaboration, H. Abramowicz et al., Measurement of beauty production in DIS and $F_{2}^{b \bar{b}}$ extraction at ZEUS, Eur. Phys. J. C 69 (2010) 347 [arXiv: 1005.3396] [InSPIRE].

[28] ZEUS collaboration, H. Abramowicz et al., Measurement of $D^{+}$and $\Lambda_{c}^{+}$production in deep inelastic scattering at HERA, JHEP 11 (2010) 009 [arXiv: 1007.1945] [INSPIRE].

[29] ZEUS collaboration, H. Abramowicz et al., Measurement of beauty production in deep inelastic scattering at HERA using decays into electrons, Eur. Phys. J. C 71 (2011) 1573 [arXiv: 1101.3692] [INSPIRE].

[30] ZEUS collaboration, H. Abramowicz et al., Measurement of $D^{ \pm}$production in deep inelastic ep scattering with the ZEUS detector at HERA, JHEP 05 (2013) 023 [arXiv: 1302 .5058] [INSPIRE].

[31] ZEUS collaboration, H. Abramowicz et al., Measurement of $D^{* \pm}$ production in deep inelastic scattering at HERA, JHEP 05 (2013) 097 [arXiv: 1303.6578] [INSPIRE].

[32] H1 Collaboration, ZEUS collaboration, H. Abramowicz et al., Combination and QCD analysis of charm production cross section measurements in deep-inelastic ep scattering at HERA, Eur. Phys. J. C 73 (2013) 2311 [arXiv:1211.1182] [INSPIRE].

[33] ZEUS collaboration, The ZEUS detector, Status Report, DESY (1993).

[34] N. Harnew et al., Vertex triggering using time difference measurements in the ZEUS central tracking detector, Nucl. Instrum. Meth. A 279 (1989) 290 [INSPIRE].

[35] B. Foster et al., The performance of the ZEUS central tracking detector z-by-timing electronics in a transputer based data acquisition system, Nucl. Phys. Proc. Suppl.

B 32 (1993) 181.

[36] ZEUS collaboration, B. Foster et al., The design and construction of the ZEUS central tracking detector, Nucl. Instrum. Meth. A 338 (1994) 254 [INSPIRE].

[37] ZEUS collaboration, A. Polini et al., The design and performance of the ZEUS Micro Vertex detector, Nucl. Instrum. Meth. A 581 (2007) 656 [arXiv:0708.3011] [INSPIRE].

[38] M. Derrick et al., Design and construction of the ZEUS barrel calorimeter, Nucl. Instrum. Meth. A 309 (1991) 77 [inSPIRE].

[39] ZEUS Calorimeter Group, ZEUS collaboration, A. Andresen et al., Construction and beam test of the ZEUS forward and rear calorimeter, Nucl. Instrum. Meth. A 309 (1991) 101 [INSPIRE]. 
[40] A. Caldwell et al., Design and implementation of a high precision readout system for the ZEUS calorimeter, Nucl. Instrum. Meth. A 321 (1992) 356 [INSPIRE].

[41] ZEUS Barrel Calorimeter Group collaboration, A. Bernstein et al., Beam tests of the ZEUS barrel calorimeter, Nucl. Instrum. Meth. A 336 (1993) 23 [INSPIRE].

[42] J. Andruszków et al., First measurement of HERA luminosity by ZEUS lumi monitor, DESY-92-066 (1992).

[43] ZEUS collaboration, M. Derrick et al., Measurement of total and partial photon proton cross-sections at $180 \mathrm{GeV}$ center-of-mass energy, Z. Phys. C 63 (1994) 391 [INSPIRE].

[44] ZEUS Luminosity Group collaboration, J. Andruszkow et al., Luminosity measurement in the ZEUS experiment, Acta Phys. Polon. B 32 (2001) 2025 [InSPIRE].

[45] M. Helbich et al., The spectrometer system for measuring ZEUS luminosity at HERA, Nucl. Instrum. Meth. A 565 (2006) 572 [physics/0512153] [INSPIRE].

[46] H. Jung, Hard diffractive scattering in high-energy ep collisions and the Monte Carlo generator RAPGAP, Comput. Phys. Commun. 86 (1995) 147 [INSPIRE].

[47] CTEQ collaboration, H.L. Lai et al., Global QCD analysis of parton structure of the nucleon: CTEQ5 parton distributions, Eur. Phys. J. C 12 (2000) 375 [hep-ph/9903282] [INSPIRE].

[48] A. Kwiatkowski, H. Spiesberger and H.J. Mohring, Heracles: an event generator for ep interactions at HERA energies including radiative processes: version 1.0, Comput. Phys. Commun. 69 (1992) 155 [INSPIRE].

[49] G.A. Schuler and H. Spiesberger, Django: the interface for the event generators Heracles and Lepto, in Proceedings of the Workshop on Physics at HERA, W. Buchmüller and G. Ingelman eds., DESY, Hamburg, Germany (1991).

[50] L. Lönnblad, ARIADNE version 4: a program for simulation of QCD cascades implementing the color dipole model, Comput. Phys. Commun. 71 (1992) 15 [INSPIRE].

[51] T. Sjöstrand, High-energy physics event generation with PYTHIA 5.7 and JETSET \%.4, Comput. Phys. Commun. 82 (1994) 74 [INSPIRE].

[52] T. Sjöstrand et al., High-energy physics event generation with PYTHIA 6.1, Comput. Phys. Commun. 135 (2001) 238 [hep-ph/0010017] [INSPIRE].

[53] M.G. Bowler, $e^{+} e^{-}$production of heavy quarks in the string model, Z. Phys. C 11 (1981) 169 [INSPIRE].

[54] T. Sjöstrand, L. Lönnblad and S. Mrenna, PYTHIA 6.2: physics and manual, hep-ph/0108264 [INSPIRE].

[55] R. Brun et al., Geant3, CERN-DD/EE/84-1 (1987).

[56] Particle Data Group collaboration, K. Nakamura et al., Review of particle physics, J. Phys. G 37 (2010) 075021 [inSPIRE].

[57] E. Lohrmann, A summary of charm hadron production fractions, arXiv:1112.3757 [INSPIRE].

[58] O. Viazlo, Differential cross sections of charm and beauty production at large momentum transfer with the ZEUS experiment at the HERA collider, Master Thesis, Kyiv University, Kyiv, Ukraine (2012). 
[59] B.W. Harris and J. Smith, Charm quark and $D^{* \pm}$ cross-sections in deeply inelastic scattering at HERA, Phys. Rev. D 57 (1998) 2806 [hep-ph/9706334] [INSPIRE].

[60] J. Smith and W.L. van Neerven, QCD corrections to heavy flavor photoproduction and electroproduction, Nucl. Phys. B 374 (1992) 36 [inSPIRE].

[61] ZEUS collaboration, S. Chekanov et al., A ZEUS next-to-leading-order QCD analysis of data on deep inelastic scattering, Phys. Rev. D 67 (2003) 012007 [hep-ex/0208023] [INSPIRE].

[62] S. Alekhin, J. Blumlein, S. Klein and S. Moch, The 3, 4 and 5-flavor NNLO parton from deep-inelastic-scattering data and at hadron colliders, Phys. Rev. D 81 (2010) 014032 [arXiv: 0908.2766] [INSPIRE].

[63] M.R. Whalley, D. Bourilkov and R.C. Group, The Les Houches accord PDFs (LHAPDF) and LHAGLUE, hep-ph/0508110 [INSPIRE].

[64] LHAPDF, https://lhapdf.hepforge.org.

[65] S.D. Ellis and D.E. Soper, Successive combination jet algorithm for hadron collisions, Phys. Rev. D 48 (1993) 3160 [hep-ph/9305266] [INSPIRE].

[66] S. Catani, Y.L. Dokshitzer, M.H. Seymour and B.R. Webber, Longitudinally invariant $K_{t}$ clustering algorithms for hadron hadron collisions, Nucl. Phys. B 406 (1993) 187 [InSPIRE].

[67] W.H. Smith, K. Tokushuku and L.W. Wiggers, The ZEUS trigger system, in Proceedings of Computing in High-Energy Physics (CHEP), Annecy, France, C. Verkerk and

W. Wojcik eds., CERN, Geneva, Switzerland (1992) [DESY-92-150B].

[68] P. Roloff, Measurement of charm and beauty production in deep inelastic scattering at HERA, Ph.D. Thesis, Hamburg University, Hamburg, Germany (2011)

[DESY-THESIS-2011-049].

[69] H. Abramowicz, A. Caldwell and R. Sinkus, Neural network based electron identification in the ZEUS calorimeter, Nucl. Instrum. Meth. A 365 (1995) 508 [hep-ex/9505004] [INSPIRE].

[70] ZEUS collaboration, J. Breitweg et al., Measurement of the diffractive structure function $F_{2}(D(4))$ at HERA, Eur. Phys. J. C 1 (1998) 81 [hep-ex/9709021] [INSPIRE].

[71] G.M. Briskin, Diffractive dissociation in ep deep inelastic scattering, Ph.D. Thesis, Tel Aviv University, Tel Aviv, Israel (1998) [DESY-THESIS-1998-036].

[72] S. Bentvelsen, J. Engelen and P. Kooijman, Reconstruction of $\left(x, Q^{2}\right)$ and extraction of structure functions in neutral current scattering at HERA, in Proceedings of the Workshop on Physics at HERA, W. Buchmüller and G. Ingelman eds., DESY, Hamburg Germany (1992).

[73] R. Sinkus and T. Voss, Particle identification with neural networks using a rotational invariant moment representation, Nucl. Instrum. Meth. A 391 (1997) 360 [INSPIRE].

[74] F. Jacquet and A. Blondel, Detection of the charged current event - Method II, in Proceedings of the study for an ep facility for Europe, U. Amaldi ed., DESY, Hamburg Germany (1979) [DESY-79-48].

[75] B. Kahle, Measurement of beauty-production in deep inelastic scattering at HERA II, Ph.D. Thesis, Hamburg University, Hamburg, Germany (2005) [DESY-THESIS-06-011]. 
[76] K. Rose, E. Gurewitz and G.C. Fox, Statistical mechanics and phase transitions in clustering, Phys. Rev. Lett. 65 (1990) 945.

[77] K. Rose, Deterministic annealing for clustering, compression, classification, regression, and related optimization problems, IEEE Proc. 86 (1998) 2210.

[78] F. Didierjean, G. Duchêne and A. Lopez-Martens, The deterministic annealing filter: A new clustering method for $\gamma$-ray tracking algorithms, Nucl. Instrum. Meth. A 615 (2010) 188.

[79] V. Libov, Measurement of charm and beauty production in deep inelastic scattering at HERA and test beam studies of ATLAS pixel sensors, Ph.D. Thesis, Hamburg University, Hamburg, Germany (2013) [DESY-THESIS-2013-030].

[80] C. Peterson, D. Schlatter, I. Schmitt and P.M. Zerwas, Scaling violations in inclusive $e^{+} e^{-}$ annihilation spectra, Phys. Rev. D 27 (1983) 105 [INSPIRE].

[81] ZEUS collaboration, S. Chekanov et al., Measurement of the charm fragmentation function in $D^{*}$ photoproduction at HERA, JHEP 04 (2009) 082 [arXiv:0901.1210] [INSPIRE].

[82] OPAL collaboration, G. Abbiendi et al., Inclusive analysis of the b quark fragmentation function in $Z$ decays at LEP, Eur. Phys. J. C 29 (2003) 463 [hep-ex/0210031] [InSPIRE].

[83] HERA combined results, https://www.desy.de/h1zeus/combined_results/.

[84] HERA combined results, HERAPDF table, https://www.desy.de/h1zeus/combined_results/herapdftable.

[85] A.D. Martin, W.J. Stirling, R.S. Thorne and G. Watt, Parton distributions for the LHC, Eur. Phys. J. C 63 (2009) 189 [arXiv:0901.0002] [InSPIRE].

[86] P.M. Nadolsky et al., Implications of CTEQ global analysis for collider observables, Phys. Rev. D 78 (2008) 013004 [arXiv:0802.0007] [INSPIRE].

[87] R.S. Thorne and W.K. Tung, PQCD formulations with heavy quark masses and global analysis, arXiv:0809.0714 [INSPIRE].

[88] P. Jimenez-Delgado and E. Reya, Dynamical NNLO parton distributions, Phys. Rev. D 79 (2009) 074023 [arXiv:0810.4274] [INSPIRE].

[89] S. Alekhin and S. Moch, Higher order QCD corrections to charged-lepton deep-inelastic scattering and global fits of parton distributions, Phys. Lett. B 672 (2009) 166 [arXiv:0811.1412] [INSPIRE].

[90] S. Alekhin, J. Blumlein and S.-O. Moch, Update of the NNLO PDFs in the 3-, 4- and 5-flavour scheme, PoS(DIS 2010) 021 [arXiv: 1007.3657] [INSPIRE].

[91] H1 AND ZEUS collaboration, F.D. Aaron et al., Combined measurement and QCD analysis of the inclusive $e^{ \pm} p$ scattering cross sections at HERA, JHEP 01 (2010) 109 [arXiv:0911.0884] [INSPIRE].

[92] S. Alekhin, J. Blümlein, K. Daum, K. Lipka and S. Moch, Precise charm-quark mass from deep-inelastic scattering, Phys. Lett. B 720 (2013) 172 [arXiv:1212.2355] [INSPIRE].

[93] S. Alekhin, K. Daum, K. Lipka and S. Moch, Determination of the charm-quark mass in the MS-bar scheme using charm production data from deep inelastic scattering at HERA, Phys. Lett. B 718 (2012) 550 [arXiv:1209.0436] [InSPIRE].

[94] HERAFitter-0.2.1, http://projects.hepforge.org/herafitter. 
[95] S. Alekhin and S. Moch, Heavy-quark deep-inelastic scattering with a running mass, Phys. Lett. B 699 (2011) 345 [arXiv: 1011.5790] [InSPIRE].

[96] S. Alekhin and S.-O. Moch, Running heavy-quark masses in DIS, arXiv:1107.0469 [INSPIRE].

[97] S. Alekhin, OPENQCDRAD-1.5, http://www-zeuthen.desy.de/ alekhin/OPENQCDRAD

[98] N. Gray, D.J. Broadhurst, W. Grafe and K. Schilcher, Three loop relation of quark (modified) MS and pole masses, Z. Phys. C 48 (1990) 673 [INSPIRE].

[99] K.G. Chetyrkin and M. Steinhauser, The relation between the MS-bar and the on-shell quark mass at order $\alpha_{s}^{3}$, Nucl. Phys. B 573 (2000) 617 [hep-ph/9911434] [INSPIRE].

[100] K. Melnikov and T.v. Ritbergen, The three loop relation between the MS-bar and the pole quark masses, Phys. Lett. B 482 (2000) 99 [hep-ph/9912391] [INSPIRE].

[101] Particle Data Group, J. Beringer et al., Review of particle physics, Phys. Rev. D 86 (2012) 1. 


\section{The ZEUS collaboration}

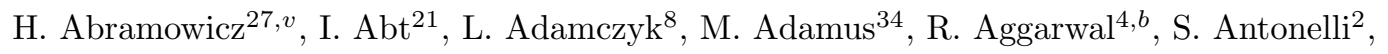
O. Arslan ${ }^{3}$, V. Aushev 16,17,p, Y. Aushev ${ }^{17, p, q}$, O. Bachynska ${ }^{10}$, A.N. Barakbaev ${ }^{15}$, N. Bartosik ${ }^{10}$, O. Behnke ${ }^{10}$, J. Behr ${ }^{10}$, U. Behrens ${ }^{10}$, A. Bertolin ${ }^{23}$, S. Bhadra ${ }^{36}$, I. Bloch ${ }^{11}$, V. Bokhonov ${ }^{16, p}$, E.G. Boos ${ }^{15}$, K. Borras $^{10}$, I. Brock ${ }^{3}$, R. Brugnera ${ }^{24}$, A. Bruni ${ }^{1}$, B. Brzozowska ${ }^{33}$, P.J. Bussey ${ }^{12}$, A. Caldwell ${ }^{21}$, M. Capua ${ }^{5}$, C.D. Catterall ${ }^{36}$, J. Chwastowski ${ }^{7, e}$, J. Ciborowski ${ }^{33, y}$, R. Ciesielski ${ }^{10, g}$, A.M. Cooper-Sarkar ${ }^{22}$, M. Corradi ${ }^{1}$, F. Corriveau ${ }^{18}$, G. D'Agostini ${ }^{26}$, R.K. Dementiev ${ }^{20}$, R.C.E. Devenish ${ }^{22}$, G. Dolinska ${ }^{10}$, V. Drugakov ${ }^{11}$, S. Dusini ${ }^{23}$, J. Ferrando ${ }^{12}$, J. Figiel ${ }^{7}$, B. Foster ${ }^{13, m}$, G. Gach ${ }^{8}$, A. Garfagnini ${ }^{24}$, A. Geiser ${ }^{10}$, A. Gizhko ${ }^{10}$, L.K. Gladilin ${ }^{20}$, O. Gogota ${ }^{17}$, Yu.A. Golubkov ${ }^{20}$, J. Grebenyuk ${ }^{10}$, I. Gregor ${ }^{10}$, G. Grzelak ${ }^{33}$, O. Gueta ${ }^{27}$,

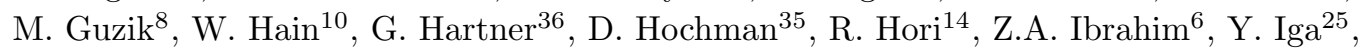
M. Ishitsuka ${ }^{28}$, A. Iudin ${ }^{17, q}$, F. Januschek ${ }^{10}$, I. Kadenko ${ }^{17}$, S. Kananov ${ }^{27}$, T. Kanno ${ }^{28}$, U. Karshon ${ }^{35}$, M. Kaur ${ }^{4}$, P. Kaur ${ }^{4, b}$, L.A. Khein ${ }^{20}$, D. Kisielewska ${ }^{8}$, R. Klanner ${ }^{13}$, U. Klein ${ }^{10, h}$, N. Kondrashova ${ }^{17, r}$, O. Kononenko ${ }^{17}$, Ie. Korol ${ }^{10}$, I.A. Korzhavina ${ }^{20}$, A. Kotański ${ }^{9}$, U. Kötz ${ }^{10}$, N. Kovalchuk ${ }^{17, s}$, H. Kowalski ${ }^{10}$, O. Kuprash ${ }^{10}$, M. Kuze ${ }^{28}$, B.B. Levchenko ${ }^{20}$, A. Levy ${ }^{27}$, V. Libov ${ }^{10}$, S. Limentani ${ }^{24}$, M. Lisovyi ${ }^{10}$, E. Lobodzinska ${ }^{10}$, W. Lohmann ${ }^{11}$, B. Löhr ${ }^{10}$, E. Lohrmann ${ }^{13}$, A. Longhin ${ }^{23, u}$, D. Lontkovskyi ${ }^{10}$, O.Yu. Lukina ${ }^{20}$, J. Maeda ${ }^{28, w}$, I. Makarenko ${ }^{10}$, J. Malka ${ }^{10}$, J.F. Martin ${ }^{31}$, S. Mergelmeyer ${ }^{3}$, F. Mohamad Idris ${ }^{6, d}$, K. Mujkic ${ }^{10, i}$,

V. Myronenko ${ }^{10, j}$, K. Nagano ${ }^{14}$, A. Nigro ${ }^{26}$, T. Nobe ${ }^{28}$, D. Notz ${ }^{10}$, R.J. Nowak ${ }^{33}$, K. Olkiewicz ${ }^{7}$, Yu. Onishchuk ${ }^{17}$, E. Paul ${ }^{3}$, W. Perlański ${ }^{33, z}$, H. Perrey ${ }^{10}$, N.S. Pokrovskiy ${ }^{15}$,

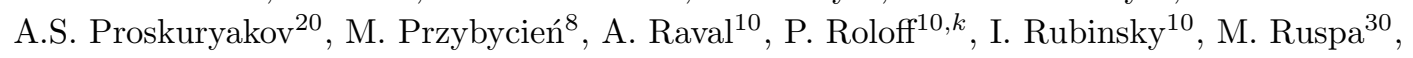
V. Samojlov ${ }^{15}$, D.H. Saxon ${ }^{12}$, M. Schioppa ${ }^{5}$, W.B. Schmidke ${ }^{21, t}$, U. Schneekloth ${ }^{10}$,

T. Schörner-Sadenius ${ }^{10}$, J. Schwartz ${ }^{18}$, L.M. Shcheglova ${ }^{20}$, R. Shehzadi ${ }^{3, a}$, R. Shevchenko ${ }^{17, q}$, O. Shkola ${ }^{17, s}$, I. Singh ${ }^{4, c}$, I.O. Skillicorn ${ }^{12}$, W. Słomiński ${ }^{9, f}$, V. Sola ${ }^{13}$, A. Solano ${ }^{29}$,

A. Spiridonov ${ }^{10, l}$, L. Stanco ${ }^{23}$, N. Stefaniuk ${ }^{10}$, A. Stern ${ }^{27}$, T.P. Stewart ${ }^{31}$, P. Stopa ${ }^{7}$, J. Sztuk-Dambietz ${ }^{13}$, D. Szuba ${ }^{13}$, J. Szuba ${ }^{10}$, E. Tassi ${ }^{5}$, T. Temiraliev ${ }^{15}$, K. Tokushuku ${ }^{14, n}$,

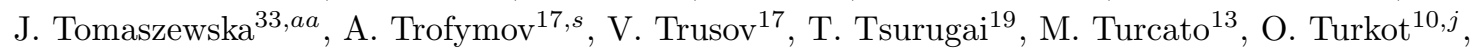
T. Tymieniecka ${ }^{34}$, A. Verbytskyi ${ }^{21}$, O. Viazlo ${ }^{17}$, R. Walczak ${ }^{22}$, W.A.T. Wan Abdullah ${ }^{6}$, K. Wichmann ${ }^{10, j}$, M. Wing ${ }^{32, x}$, G. Wolf ${ }^{10}$, S. Yamada ${ }^{14}$, Y. Yamazaki ${ }^{14, o}$, N. Zakharchuk ${ }^{17, s}$, A.F. Żarnecki ${ }^{33}$, L. Zawiejski ${ }^{7}$, O. Zenaiev ${ }^{10}$, B.O. Zhautykov ${ }^{15}$, N. Zhmak ${ }^{16, p}$, D.S. Zotkin ${ }^{20}$

1 INFN Bologna, Bologna, Italy ${ }^{A}$

2 University and INFN Bologna, Bologna, Italy ${ }^{A}$

3 Physikalisches Institut der Universität Bonn, Bonn, Germany ${ }^{B}$

4 Panjab University, Department of Physics, Chandigarh, India

5 Calabria University, Physics Department and INFN, Cosenza, Italy A

6 National Centre for Particle Physics, Universiti Malaya, 50603 Kuala Lumpur, Malaysia ${ }^{C}$

7 The Henryk Niewodniczanski Institute of Nuclear Physics, Polish Academy of Sciences, Krakow, Poland ${ }^{D}$

8 AGH-University of Science and Technology, Faculty of Physics and Applied Computer Science, Krakow, Poland ${ }^{D}$

9 Department of Physics, Jagellonian University, Cracow, Poland

10 Deutsches Elektronen-Synchrotron DESY, Hamburg, Germany

11 Deutsches Elektronen-Synchrotron DESY, Zeuthen, Germany

12 School of Physics and Astronomy, University of Glasgow, Glasgow, United Kingdom ${ }^{E}$

13 Hamburg University, Institute of Experimental Physics, Hamburg, Germany ${ }^{F}$

14 Institute of Particle and Nuclear Studies, KEK, Tsukuba, Japan ${ }^{G}$

15 Institute of Physics and Technology of Ministry of Education and Science of Kazakhstan, Almaty, Kazakhstan 
Institute for Nuclear Research, National Academy of Sciences, Kyiv, Ukraine

17 Department of Nuclear Physics, National Taras Shevchenko University of Kyiv, Kyiv, Ukraine

18 Department of Physics, McGill University, Montréal, Québec, Canada H3A 2T8 ${ }^{H}$

19 Meiji Gakuin University, Faculty of General Education, Yokohama, Japan ${ }^{G}$

20 Lomonosov Moscow State University, Skobeltsyn Institute of Nuclear Physics, Moscow, Russia ${ }^{I}$

21 Max-Planck-Institut für Physik, München, Germany

22 Department of Physics, University of Oxford, Oxford, United Kingdom ${ }^{E}$

${ }^{23}$ INFN Padova, Padova, Italy ${ }^{A}$

24 Dipartimento di Fisica dell' Università and INFN, Padova, Italy ${ }^{A}$

25 Polytechnic University, Tokyo, Japan ${ }^{G}$

${ }^{26}$ Dipartimento di Fisica, Università 'La Sapienza' and INFN, Rome, Italy ${ }^{A}$

27 Raymond and Beverly Sackler Faculty of Exact Sciences, School of Physics, Tel Aviv University, Tel Aviv, Israel ${ }^{J}$

28 Department of Physics, Tokyo Institute of Technology, Tokyo, Japan ${ }^{G}$

29 Università di Torino and INFN, Torino, Italy ${ }^{A}$

30 Università del Piemonte Orientale, Novara, and INFN, Torino, Italy ${ }^{A}$

31 Department of Physics, University of Toronto, Toronto, Ontario, Canada M5S $1 \mathrm{~A}^{\mathrm{H}}$

${ }^{32}$ Physics and Astronomy Department, University College London, London, United Kingdom ${ }^{E}$

33 Faculty of Physics, University of Warsaw, Warsaw, Poland

34 National Centre for Nuclear Research, Warsaw, Poland

35 Department of Particle Physics and Astrophysics, Weizmann Institute, Rehovot, Israel

36 Department of Physics, York University, Ontario, Canada M3J 1 P3 ${ }^{H}$

A supported by the Italian National Institute for Nuclear Physics (INFN)

$B$ supported by the German Federal Ministry for Education and Research (BMBF), under contract No. 05 H09PDF

$C$ supported by HIR grant UM.C/625/1/HIR/149 and UMRG grants RU006-2013, RP012A-13AFR and RP012B-13AFR from Universiti Malaya, and ERGS grant ER004-2012A from the Ministry of Education, Malaysia

$D$ supported by the National Science Centre under contract No. DEC-2012/06/M/ST2/00428

E supported by the Science and Technology Facilities Council, UK

$F$ supported by the German Federal Ministry for Education and Research (BMBF), under contract No. 05h09GUF, and the SFB 676 of the Deutsche Forschungsgemeinschaft (DFG)

$G$ supported by the Japanese Ministry of Education, Culture, Sports, Science and Technology (MEXT) and its grants for Scientific Research

${ }^{H}$ supported by the Natural Sciences and Engineering Research Council of Canada (NSERC)

I supported by RF Presidential grant $N$ 3042.2014.2 for the Leading Scientific Schools and by the Russian Ministry of Education and Science through its grant for Scientific Research on High Energy Physics

J supported by the Israel Science Foundation

a now at University of the Punjab, Lahore, Pakistan

${ }^{b}$ also funded by Max Planck Institute for Physics, Munich, Germany

${ }^{c}$ also funded by Max Planck Institute for Physics, Munich, Germany, now at Sri Guru Granth Sahib World University, Fatehgarh Sahib

$d$ also at Agensi Nuklear Malaysia, 43000 Kajang, Bangi, Malaysia

e also at Cracow University of Technology, Faculty of Physics, Mathematics and Applied Computer Science, Poland

${ }^{f}$ partially supported by the Polish National Science Centre projects DEC-2011/01/B/ST2/03643 and DEC-2011/03/B/ST2/00220

$g$ now at Rockefeller University, New York, NY 10065, USA

${ }^{h}$ now at University of Liverpool, United Kingdom 
i also affiliated with University College London, UK

${ }^{j}$ supported by the Alexander von Humboldt Foundation

${ }^{k}$ now at CERN, Geneva, Switzerland

$l$ also at Institute of Theoretical and Experimental Physics, Moscow, Russia

$m$ Alexander von Humboldt Professor; also at DESY and University of Oxford

$n$ also at University of Tokyo, Japan

- now at Kobe University, Japan

$p$ supported by DESY, Germany

$q$ member of National Technical University of Ukraine, Kyiv Polytechnic Institute, Kyiv, Ukraine

$r$ now at DESY ATLAS group

$s$ member of National University of Kyiv - Mohyla Academy, Kyiv, Ukraine

${ }^{t}$ now at BNL, USA

u now at LNF, Frascati, Italy

$v$ also at Max Planck Institute for Physics, Munich, Germany, External Scientific Member

$w$ now at Tokyo Metropolitan University, Japan

$x$ also supported by DESY

$y$ also at Łódź University, Poland

$z$ member of Eódź University, Poland

a now at Polish Air Force Academy in Deblin 